\title{
Water-Level Data for Las Vegas Valley, Clark County, Nevada, 1986-87
}

By Douglas K. Maurer

\section{U.S. GEOLOGICAL SURVEY}

Open-File Report 88-200

Prepared in cooperation with the

LAS VEGAS VALLEY WATER DISTRICT

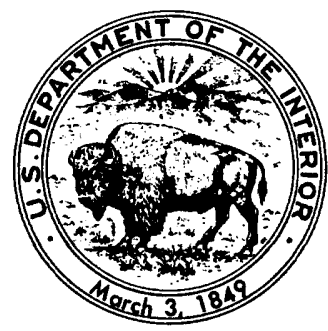

Carson City, Nevada 
DEPARTMENT OF THE INTERIOR

MANUEL LUJAN, JR., Secretary

\section{U.S. GEOLOGICAL SURVEY}

Dallas L. Peck, Director

For additional information write to:

U.S. Geological Survey

Room 227, Federal Building

705 North Plaza Street

Carson City, NV 89701
Copies of this report may be purchased from:

U.S. Geological Survey

Books and Open-File Reports Section

Federal Center, Building 810

Box 25425

Denver, CO 80225 


\section{CONTENTS}

Page

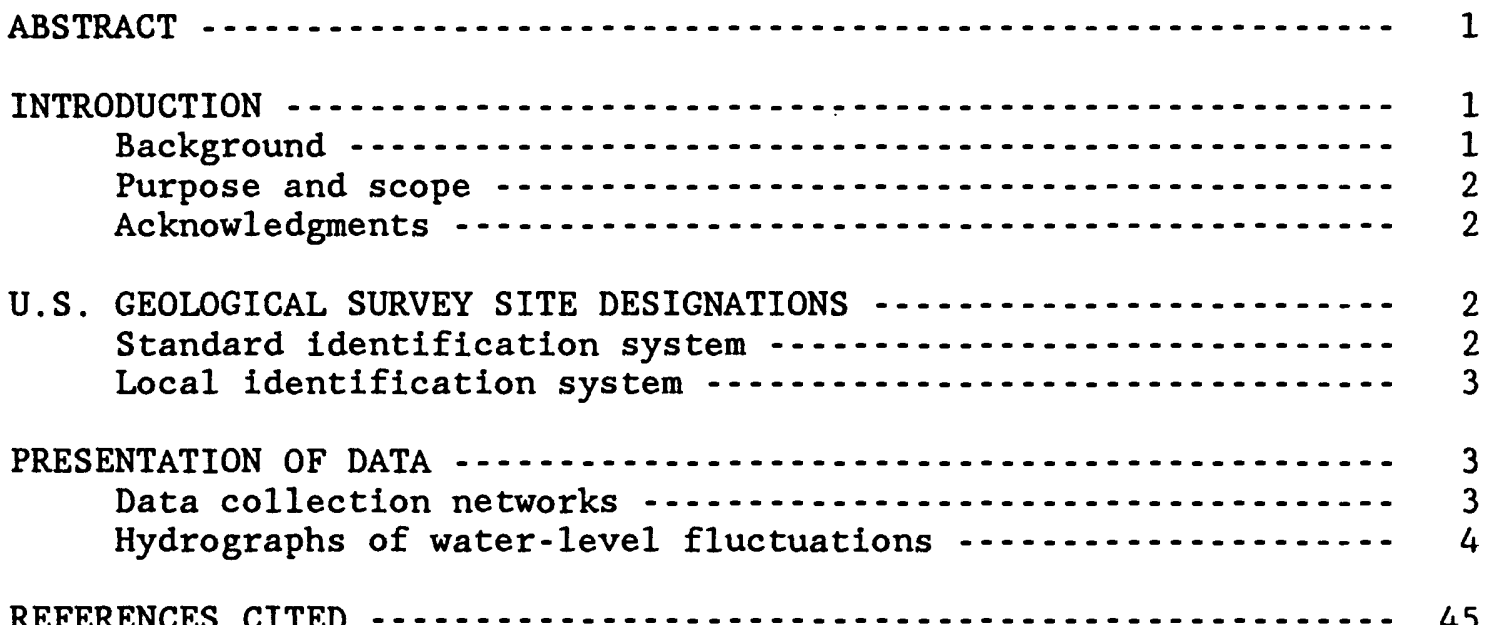




\section{ILLUSTRATIONS}

[Plates are in pocket at back of report.]

Plate 1. Map showing location of observation wells and major public-supply wells

2. Hydrographs of three wells equipped with recorders, April 1986 through April 1987

Page

Figure 1. Hydrographs of selected wells measured about weekly:

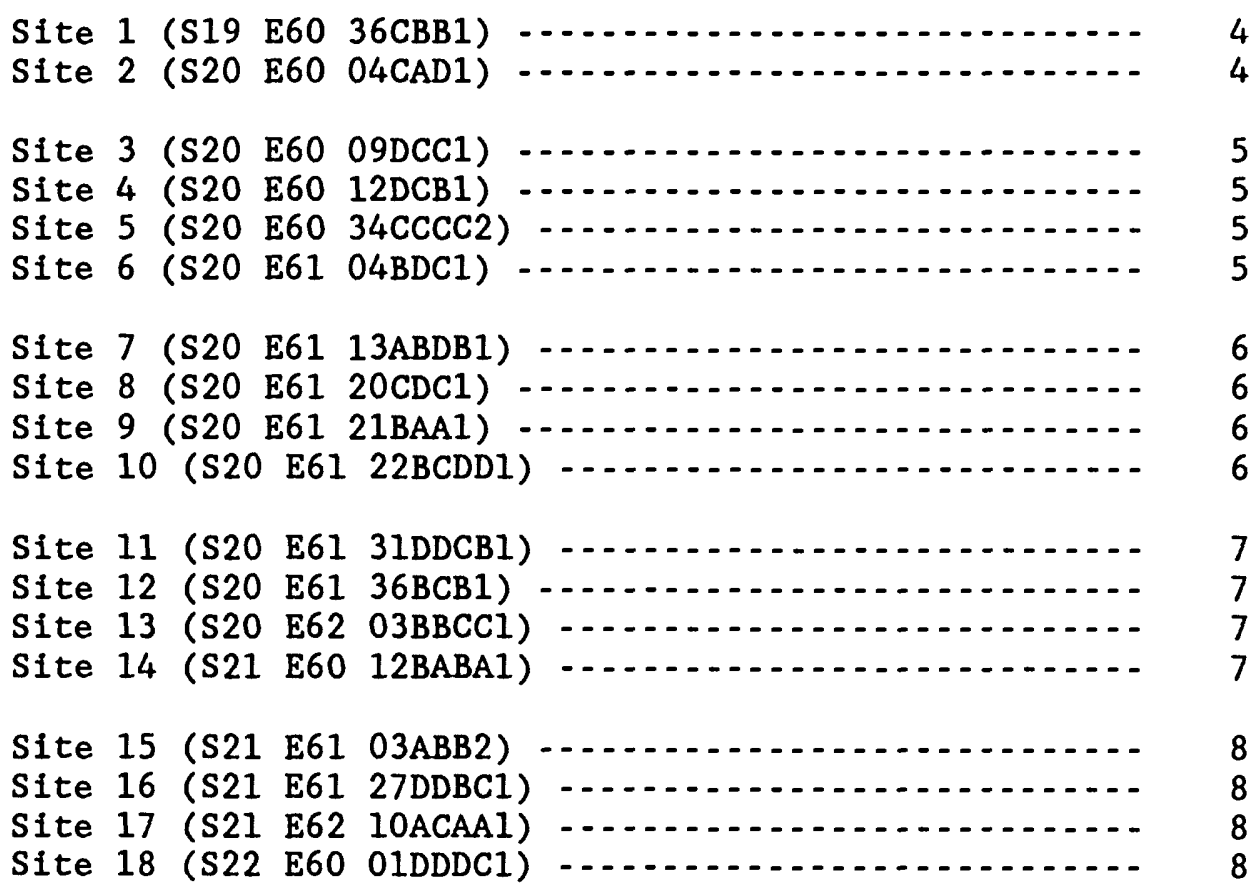




\section{TABLES}

Page

Table 1. Water-level data for wells measured about weekly $\ldots . . . . . .9$

2. Water-level data for wells measured about quarterly $\ldots . . . .34$

3. Daily mean water levels for three wells equipped

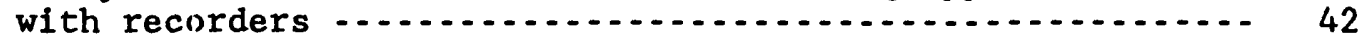

CONVERSION FACTORS AND ABBREVIATIONS

"Inch-pound" units of measure used in this report may be converted to metric (International system) units by using the following factors:

Multiply

Acre-foot per year

(acre-ft/yr)

Foot ( $f t$ )

Square mile $\left(\mathrm{mi}^{2}\right)$
By

1,233

0.3048

2.590
To obtain

Cubic meter per year $\left(\mathrm{m}^{3} / \mathrm{yr}\right)$

Meter (m)

Square kilometer $\left(\mathrm{km}^{2}\right)$

\section{ALTITUDE DATUM}

In this report, "sea level" refers to the National Geodetic Vertical Datum of 1929 (NGVD of 1929), which is derived from a general adjustment of the first-order leveling networks of both the United States and Canada. 
WATER-LEVEL DATA FOR LAS VEGAS VALLEY, CLARK COUNTY, NEVADA, 1986-87

By Douglas K. Maurer

\begin{abstract}
Tabulations of water-level measurements at 112 wells in Las Vegas Valley, Nevada, and selected hydrographs are included in this report. The measurements were made from March 1986 through April 1987. The data were collected to provide a data base to test the short-term calibration and potential re-calibration of an existing ground-water flow model of the basin. Seasonal water-level declines as great as 30 feet were measured near areas of major pumping.
\end{abstract}

\title{
INTRODUCTION
}

\section{Background}

Las Vegas Valley depends upon ground water (about 70,000 acre-ft/yr) and importation of Lake Mead water (about 130,000 acre-ft/yr) for its water supply. At the present time (1986-87), ground-water withdrawals exceed recharge, resulting in water-level declines in areas of concentrated pumpage. Current management of water use in the basin results in seasonal stress of the ground-water aquifers. During winter months, when demand is low, Lake Mead can provide most of the water; however, during summer months, when demand is high, large ground-water withdrawals occur. Principal use is by the Las Vegas Valley Water District, the City of North Las Vegas, and Nellis Air Force Base.

Las Vegas Valley encompasses about $1,564 \mathrm{mi}^{2}$ in Clark County, Nev. Boundaries and general features of the valley are shown on plate 1. The Las Vegas Valley ground-water basin is composed of unconsolidated and semiconsolidated deposits that fill the structural depression underlying the valley (Plume, 1984). These deposits are grouped into three generalized hydrologic units (David S. Morgan and Michael D. Dettinger, U.S. Geological Survey, written communication, 1987): (1) the nearsurface reservoir extending from near land surface to depths of about 200 feet, (2) the principal aquifers extending from depths of about 200 to 1,000 feet, and (3) the deep zone extending from depths of about 1,000 feet down to the underlying consolidated rock. 


\section{Purpose and Scope}

A numerical ground-water model was recently developed and calibrated to historical changes in pumpage and water levels, to evaluate the long term consequences of changes in management of the ground-water basin (David S. Morgan and Michael D. Dettinger, U.S. Geological Survey, written communication, 1987). The model will be useful for appraising the effects of long-term management strategies; however, data existing at the time of calibration were not adequate for calibrating the model to simulate the ground-water systern under rapidly changing conditions. Potential changes in water management in the valley include decreased pumpage and increased use of Lake Mead water on a time scale not represented in the calibration of the existing model.

The purpose of this study was to collect detailed water-level records adequate to provide a data base for testing the short-term calibration of the ground-water model and for re-calibrating the model if necessary. The study was funded cooperatively by the Las Vegas Valley Water District and the U.S. Geological Survey. This report is a compilation of water-level data collected at 112 wells at approximately quarterly, weekly, and hourly intervals, and presents hydrographs of water-level fluctuations recorded during the study period.

\section{Acknowledgments}

The author thanks the Clark County Department of Comprehensive Planning for providing personnel to measure water levels, and Terry Katzer and Kay Brothers of the Las Vegas Valley Water District for their assistance in measuring water levels.

\section{U.S. GEOLOGICAL SURVEY SITE DESIGNATIONS \\ Standard Identification System}

Sites are identified by the standard Geological Survey identification (ID), which is based on the grid system of latitude and longitude. The ID indicates the geographic location of each site, and provides a unique number for each. The ID consists of 15 digits: The first 6 denote the degrees, minutes, and seconds of latitude; the next 7 denote degrees, minutes, and seconds of longitude; and the last 2 digits (assigned sequentially) identify the sites within a 1 -second grid. For example, site 361611115151301 is at $36^{\circ} 16^{\prime} 11^{\prime \prime}$ latitude and $115^{\circ} 15^{\prime} 13^{\prime \prime}$ longitude, and it is the first site recorded in that 1 -second grid. 


\section{Local Identification System}

The local site-identification system used in this report is based on an index of hydrographic areas in Nevada (Rush, 1968) and the rectangular subdivision of the public lands referenced to the Mount Diablo base line and meridian. Each site designation consists of four units separated by spaces: The first unit is the hydrographic area number. The second unit is the township, preceded by an $N$ or $S$ to indicate location north or south of the base line. The third unit is the range, preceded by an $E$ to indicate location east of the meridian. The fourth unit consists of the section number and letters designating the quarter section, quarterquarter section, and so on ( $A, B, C$, and $D$ indicate the northeast, northwest, south-west, and southeast quarters, respectively), followed by a number indicating the sequence in which the site was recorded. For example, site 212 S19 E60 27BDCl is in Las Vegas Valley (hydrographic area 212). It is the first site recorded in the SW $1 / 4$ of the $S E 1 / 4$ of the NW $1 / 4$ of section 27, Township 19 South, Range 60 East, Mount Diablo base line and meridian.

\section{PRESENTATION OF DATA}

\section{Data Collection Networks}

Under cooperative agreement, the State of Nevada and the U.S. Geological Survey have made water-level measurements on two networks: one consisting of 42 wells measured annually and another consisting of 41 wells measured quarterly. For this study, both networks were combined and water levels were measured quarterly (see plate 1). Of these wells, 51 represent water levels in the principal aquifer, and 32 represent water levels in the nearsurface reservoir.

An additional 29 wells were selected for measurement on a weekly interval ( $\mathrm{plate} 1$ ). These wells were selected from existing networks and older observation networks that had been measured from 1971 to 1981 . of the wells measured weekly, 21 represent water levels in the principal aquifer, 1 represents water levels in the near-surface reservoir, and 7 represent water levels in the deep zone. Data collected from wells in the weekly and quarterly networks are presented in tables 1 and 2, respectively.

Five of the wells were equipped with hourly water-level recorders to determine how accurately the weekly measurements define the short-term water-level fluctuations. Usable records were obtained at three of the five sites. At the fourth site, the recorder malfunctioned; and at the fifth site, the well went dry after only 3 weeks of recorder operation. Data from the three hourly recorders are presented in hydrographs on plate 2 and in table 3. 


\section{Hydrographs of Water-Level Fluctuations}

The hydrographs in figure 1 show water-level fluctuations observed in wells at 18 sites measured on a weekly interval (note that vertical scales are variable to show maximum detail). These hydrographs were selected to give the reader a visual representation of water-level changes throughout the valley during the study period. Most of the data show a sinusoidal trend as water levels declined from April through September, after which most wells began to recover. Water-level declines are greatest near areas of major pumping (plate 1) with the maximum being about 30 feet (figure 1, site 11) near the well field of Las Vegas Valley Water District. Some wells show almost no water-level change (figure 1 , site 4 ) or a declining trend (figure 1 , sites 6 and 18). Site 14 (figure 1), a shallow well finished in the near-surface reservoir, shows a rising trend of about 15 feet during the study period.

Plate 2 shows water-level fluctuations recorded hourly at sites 11 , 13, and 16. The hydrographs on plate 2 correspond to hydrographs of weekly measurements of the same wells shown in figure 1. Hydrographs for sites 11 and 13, near areas of major pumping, show water-level fluctuations missed by weekly measurement. However, the hydrograph for site 16, a well distant from pumping, does not differ significantly from the hydrograph of weekly measurements.
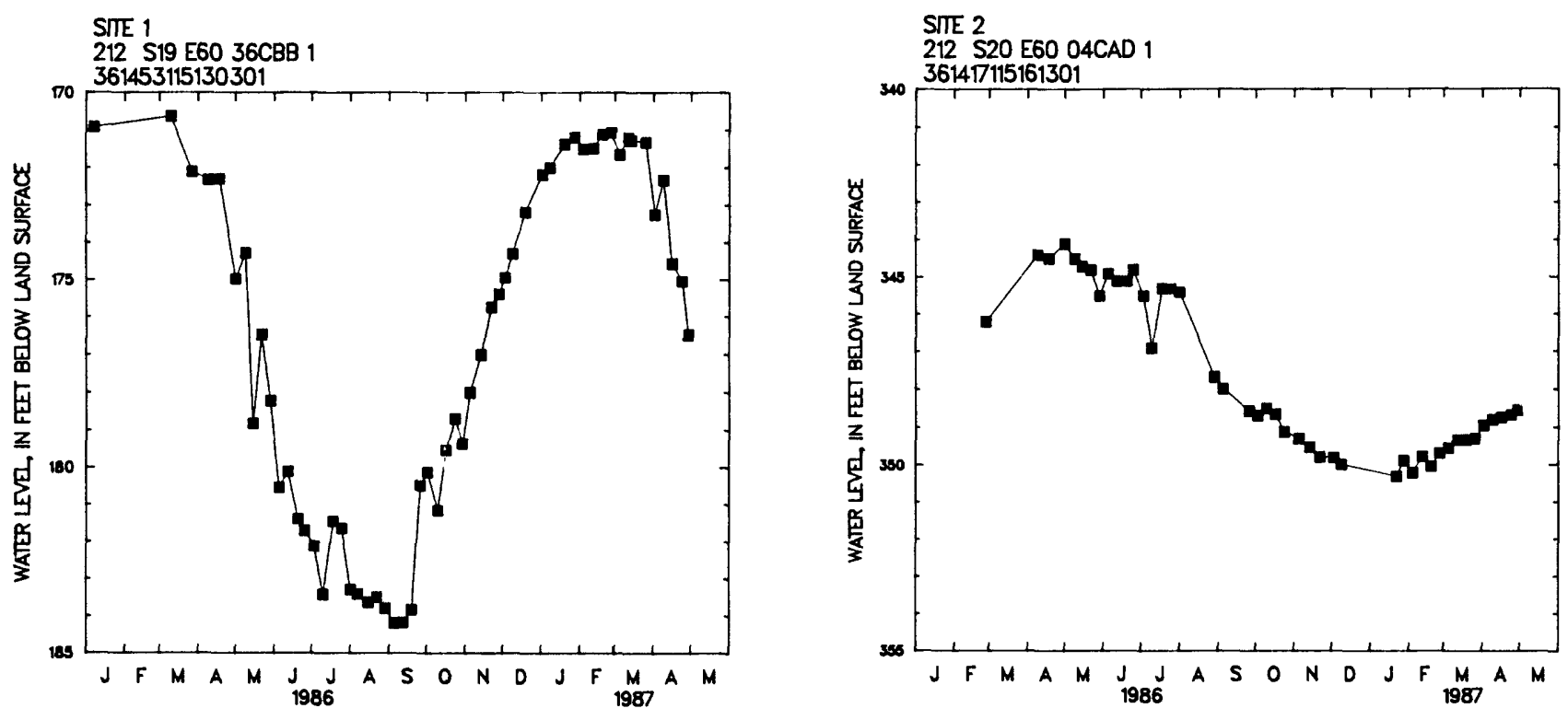

FIGURE 1.--Hydrographs of selected wells measured about weekly. 

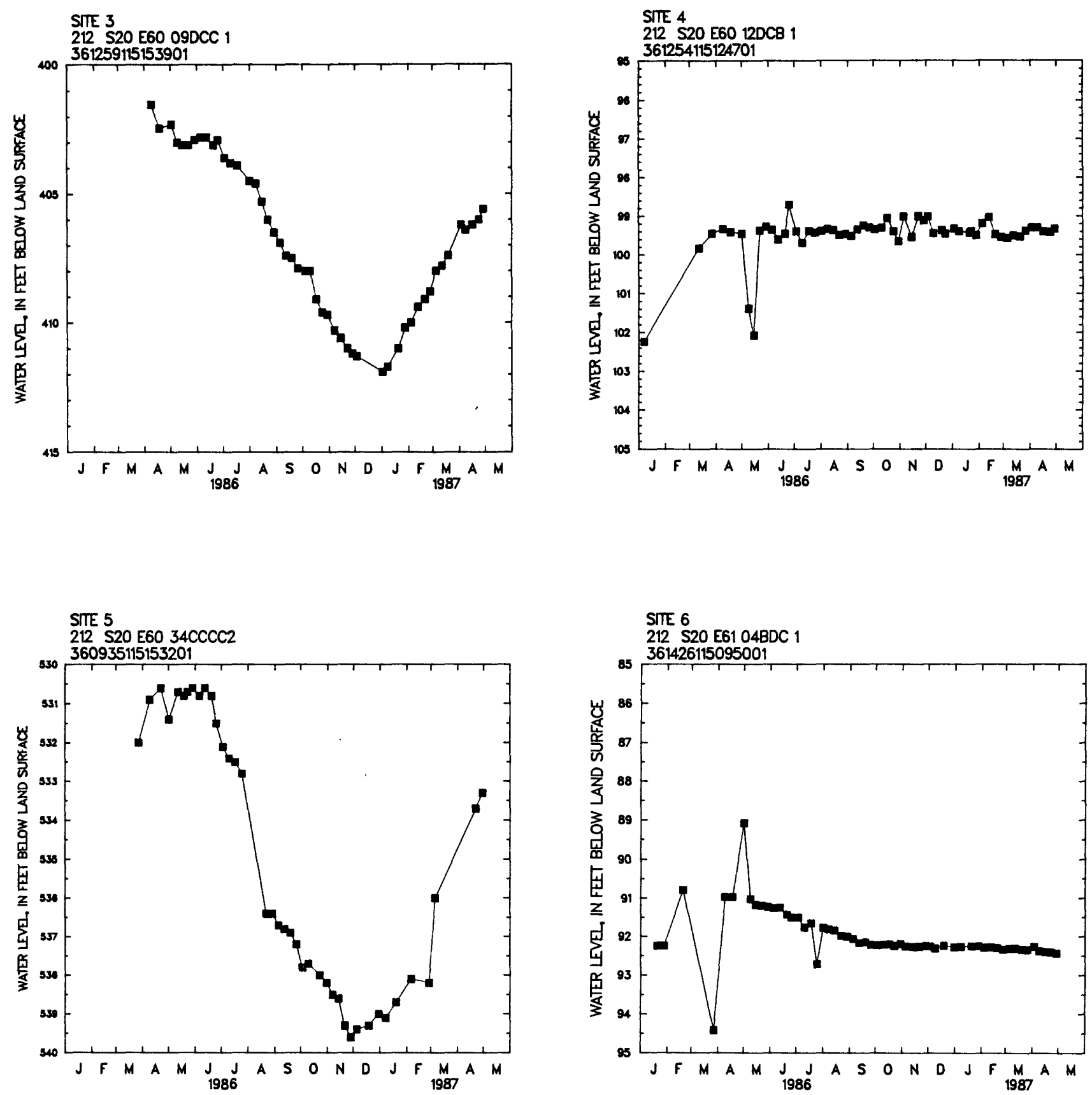

FIGURE 1.--Continued. 

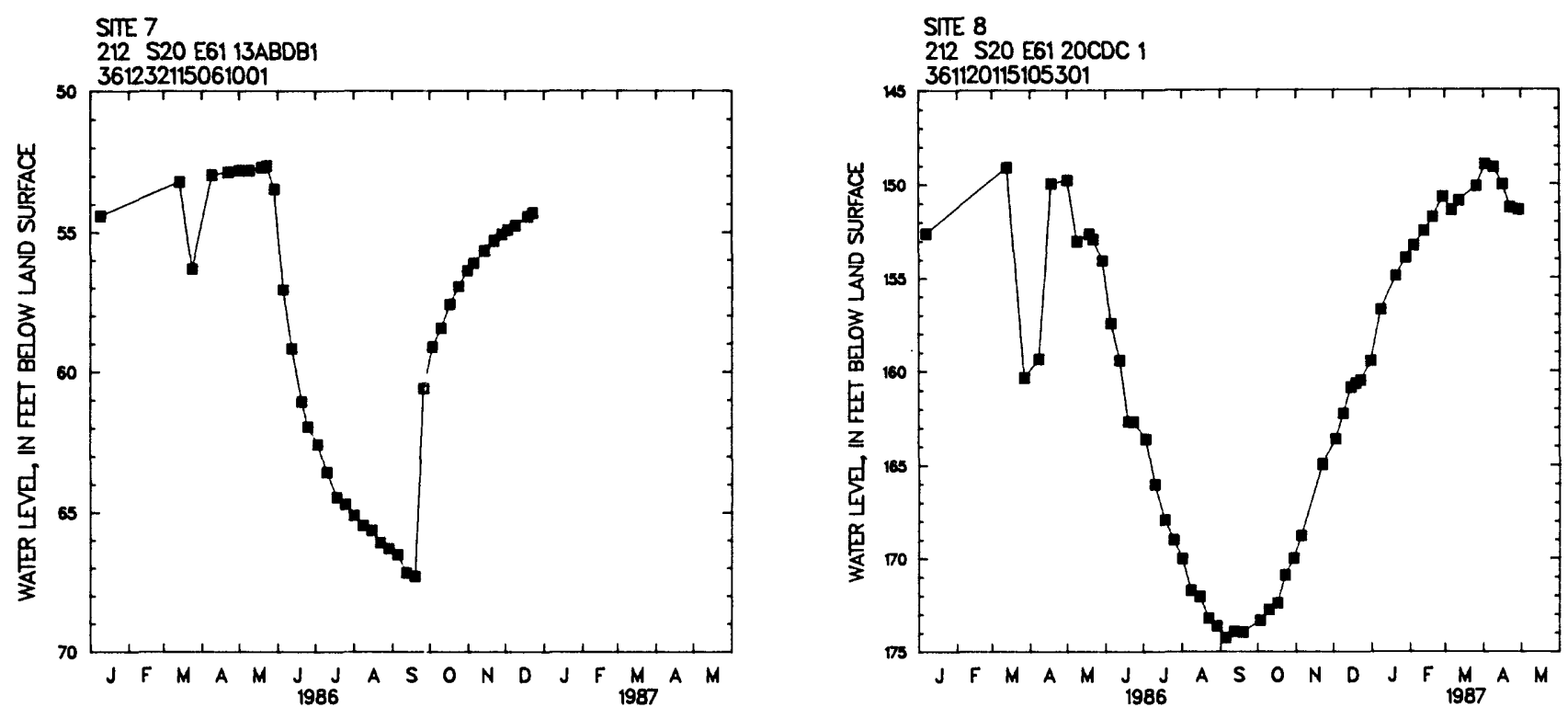

SIIE 9

212 S20 E61 21BAA 1

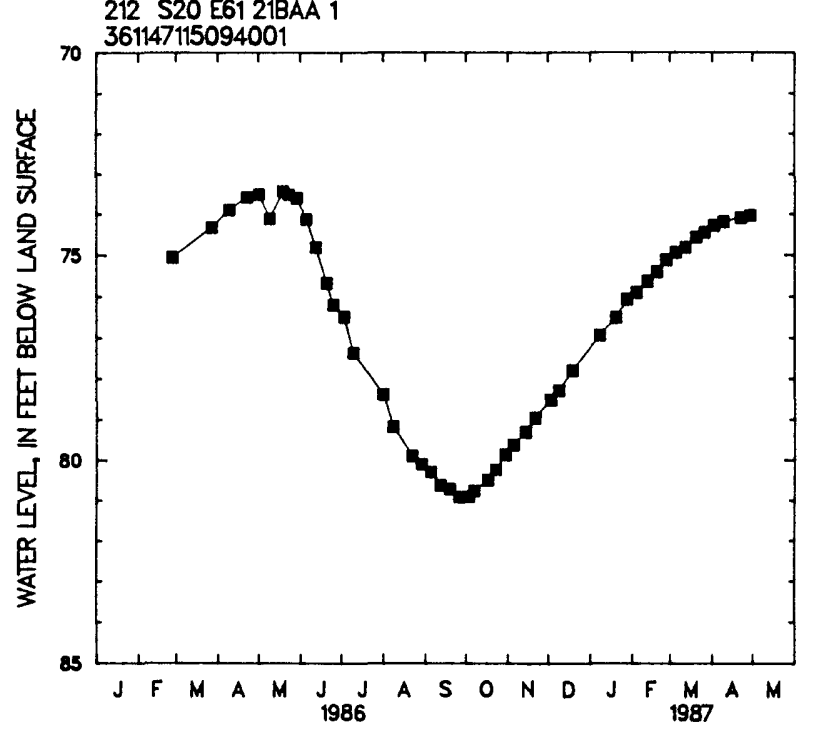

STाE 10

212 S20 E61 22BCDD1

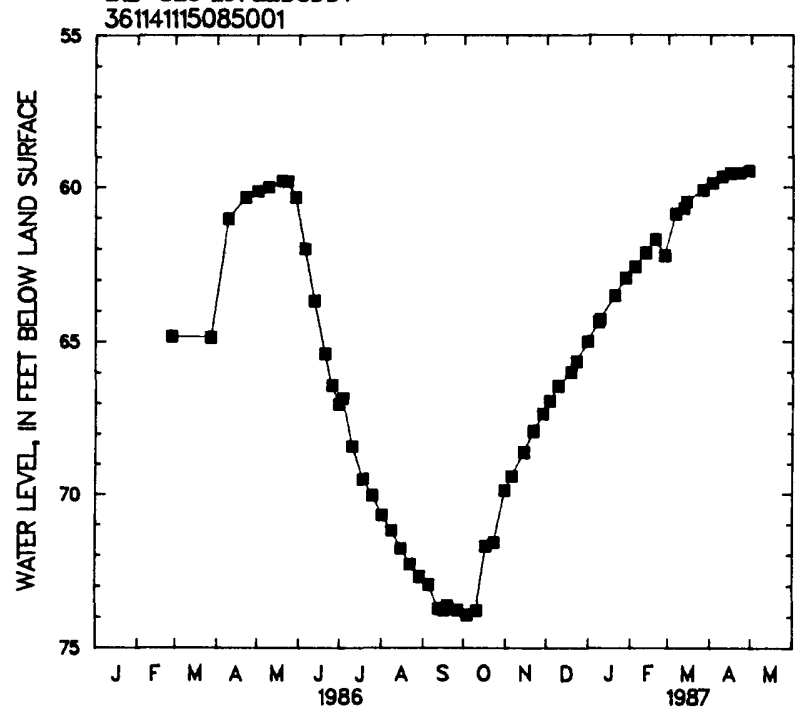

FIGURE 1.--Continued. 

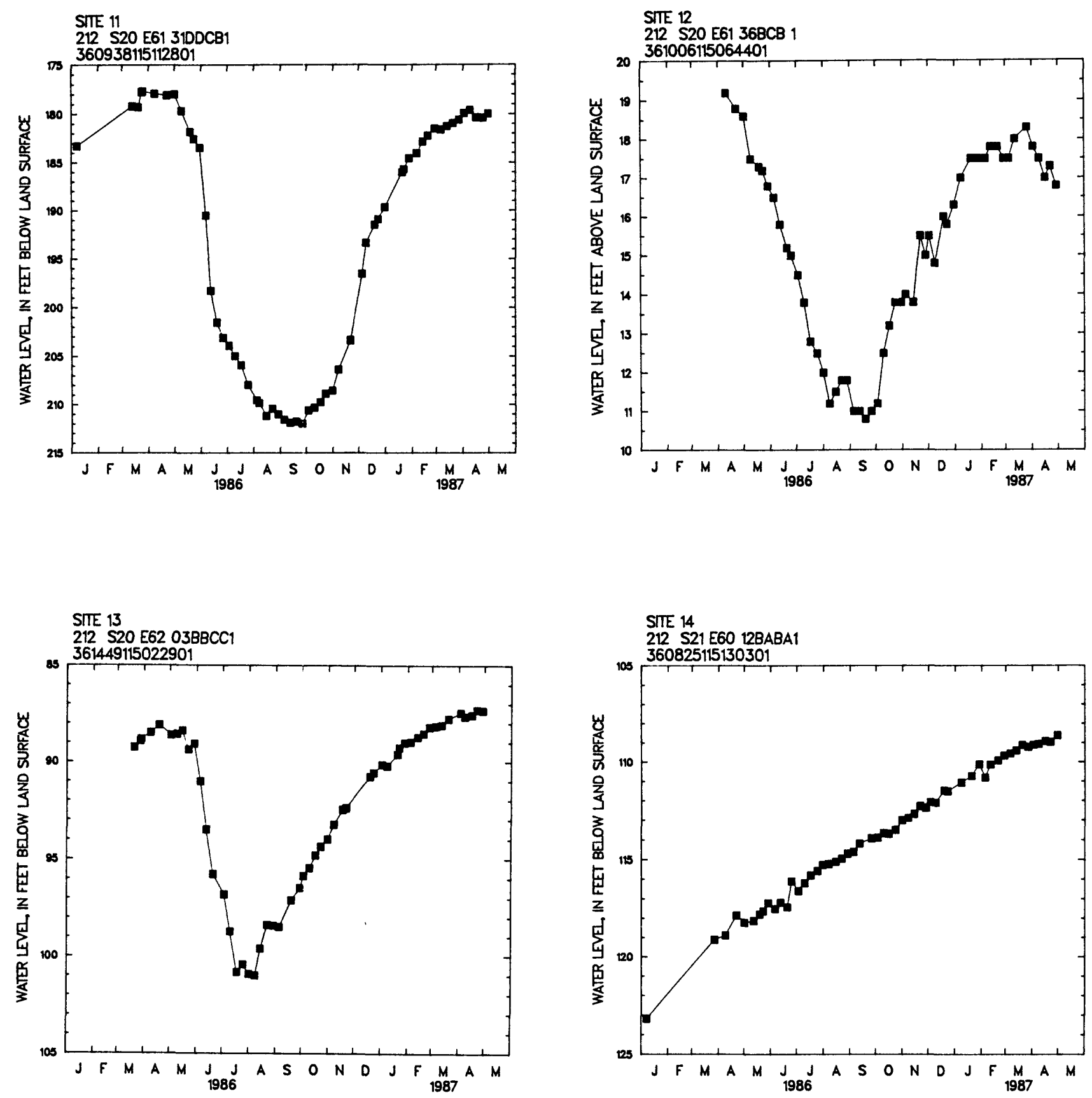

FIGURE 1..-Continued. 

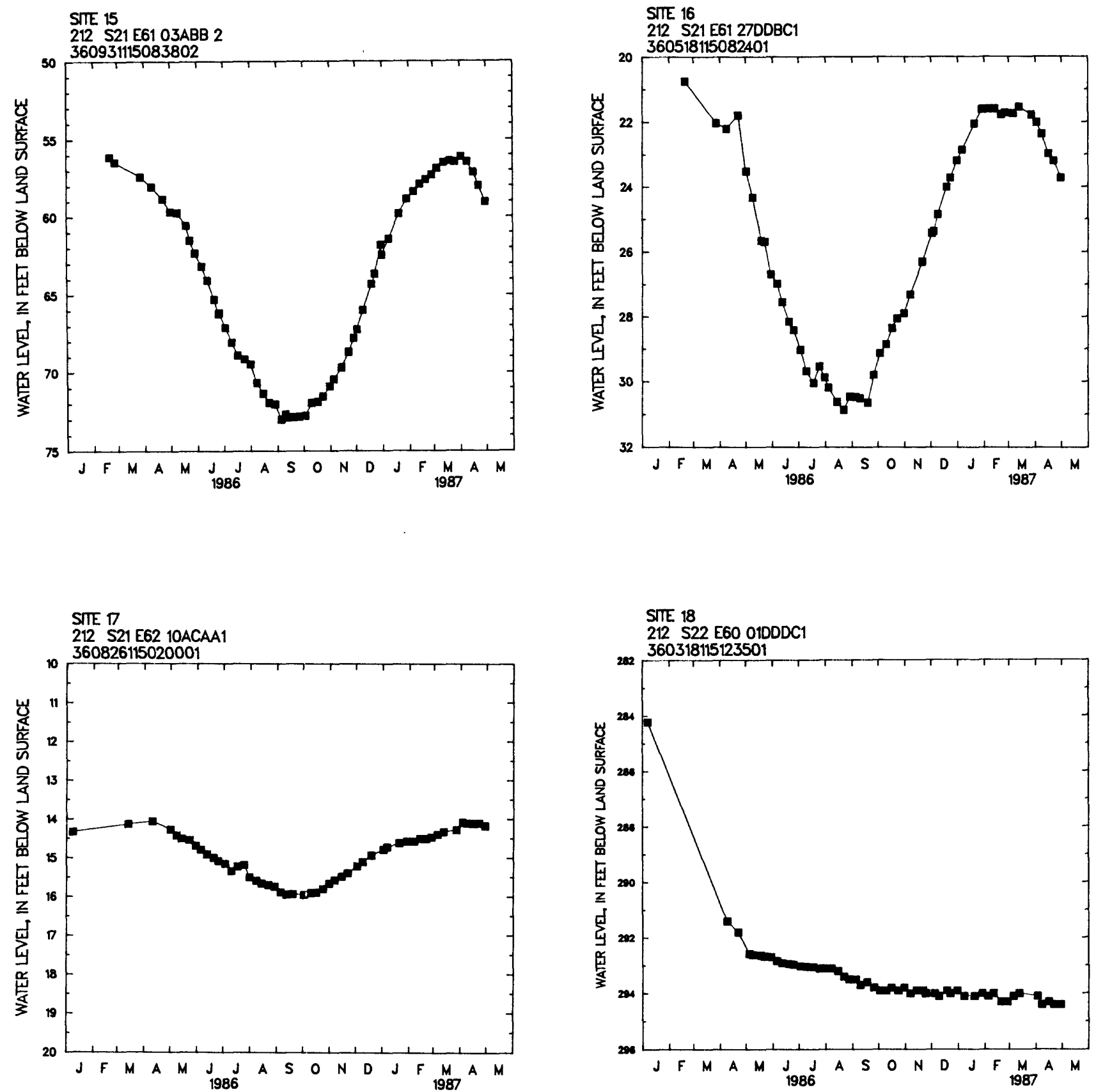

FIGURE 1.--Continued. 
TABLE 1.--Water-level data for wells measured about weekly

$$
[\cdots, \text { no data available }]
$$

Site designations.--USGS identifications: see text section titled "U.S. Geological Survey Site

Designations." Well owner abbreviations: City N.L.V., City of North Las Vegas; L.V. Union School Dist., Las Vegas Union School District; LVWW, Las Vegas Valley Water District; U.S.A.F., United States Air Force; USGS, United States Geological Survey; Union Pacific R.R., Union Pacific Railroad.

Water use.--Abbreviations: $H$, domestic; $P$, public supply; U, unused.

Water-level depth.--Minus sign indicates water level above land surface.

Water-level measurement method.--Abbreviations: A, air line; G, pressure gage; S, steel tape;

$T$, electric tape.

\begin{tabular}{|c|c|c|c|c|c|c|c|c|}
\hline \multicolumn{2}{|c|}{ site designations } & \multirow{2}{*}{\multicolumn{2}{|c|}{$\begin{array}{c}\text { Land- } \\
\text { surface } \\
\text { altitude } \\
\text { (feet } \\
\text { above } \\
\text { Water sea } \\
\text { use level) }\end{array}$}} & \multirow{2}{*}{$\begin{array}{c}\text { Depth } \\
\text { of } \\
\text { well } \\
\text { (feet) }\end{array}$} & \multirow[b]{2}{*}{$\begin{array}{l}\text { Depth to } \\
\text { shal lowest } \\
\text { opening } \\
\text { (feet) }\end{array}$} & \multicolumn{3}{|c|}{ Water level } \\
\hline $\begin{array}{l}\text { Local USGS } \\
\text { identification; } \\
\text { well name or number; } \\
\text { and owner }\end{array}$ & $\begin{array}{c}\text { Standard } \\
\text { USGS } \\
\text { ident ification }\end{array}$ & & & & & Date & $\begin{array}{l}\text { Depth } \\
\text { (feet below } \\
\text { land surface) }\end{array}$ & $\begin{array}{l}\text { Measure- } \\
\text { ment } \\
\text { method }\end{array}$ \\
\hline
\end{tabular}

U.S. Geological Survey Measurements

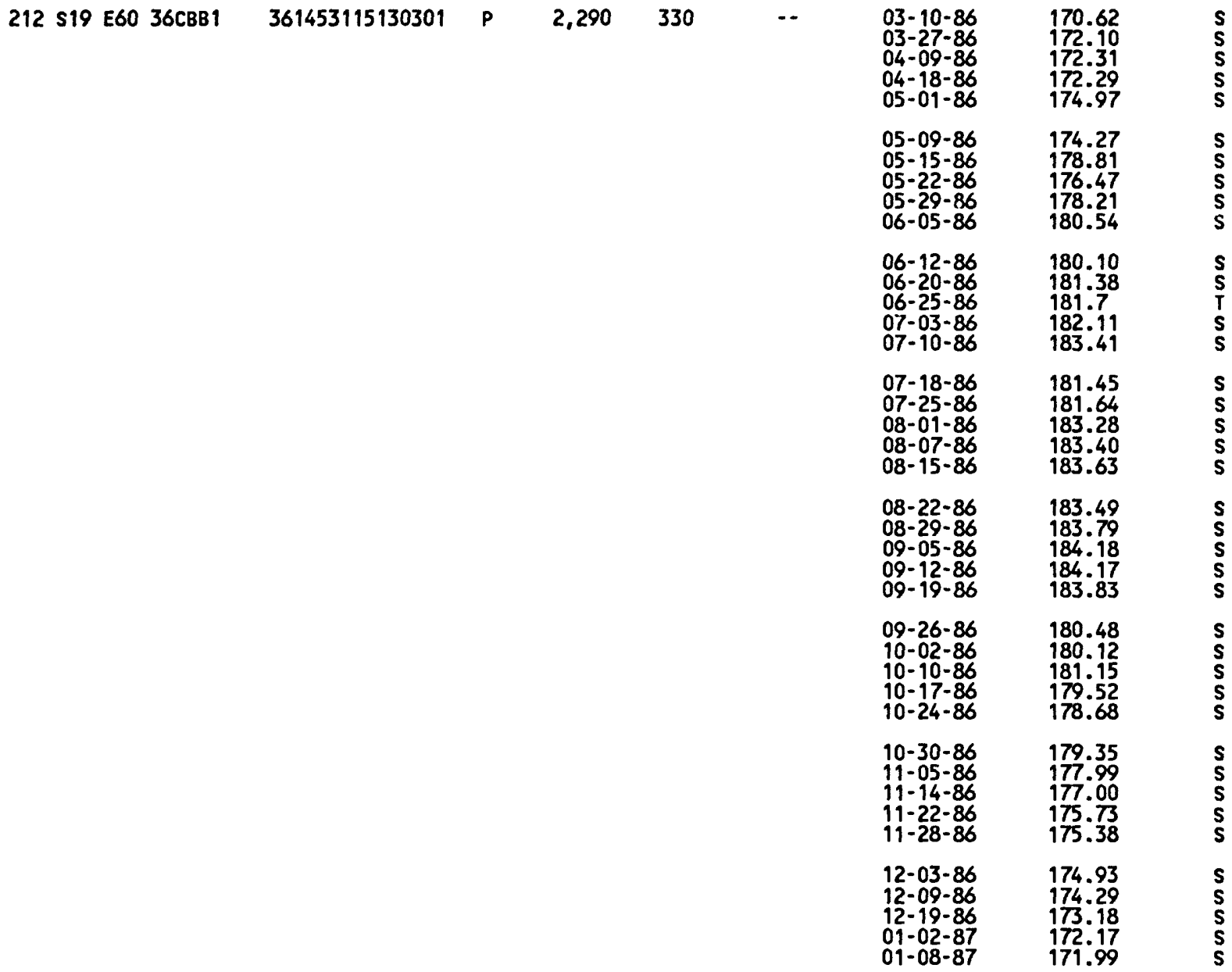


TABLE 1.--Water-level data for wells measured about weekly--Continued.

\begin{tabular}{|c|c|c|c|c|c|c|c|c|}
\hline \multicolumn{2}{|c|}{ Site designations } & \multirow{2}{*}{\multicolumn{2}{|c|}{$\begin{array}{c}\text { Land- } \\
\text { surface } \\
\text { altitude } \\
\text { (feet } \\
\text { above } \\
\text { Water sea } \\
\text { use level) }\end{array}$}} & \multirow[b]{2}{*}{$\begin{array}{c}\text { Depth } \\
\text { of } \\
\text { well } \\
\text { (feet) }\end{array}$} & \multirow[b]{2}{*}{$\begin{array}{l}\text { Depth to } \\
\text { shallowest } \\
\text { opening } \\
\text { (feet) }\end{array}$} & \multicolumn{3}{|c|}{ Water level } \\
\hline $\begin{array}{l}\text { Local USGS } \\
\text { identification; } \\
\text { well name or number; } \\
\text { and owner }\end{array}$ & $\begin{array}{l}\text { Standard } \\
\text { USGS } \\
\text { ident ification }\end{array}$ & & & & & Date & $\begin{array}{l}\text { Depth } \\
\text { (feet below } \\
\text { land surface) }\end{array}$ & $\begin{array}{l}\text { Measure- } \\
\text { ment } \\
\text { method }\end{array}$ \\
\hline \multirow{3}{*}{\multicolumn{2}{|c|}{212 S19 E60 36CBB1--Cont inued }} & & & & & $\begin{array}{l}01-20-87 \\
01-28-87 \\
02-04-87 \\
02-12-87 \\
02-19-87\end{array}$ & $\begin{array}{l}171.36 \\
171.18 \\
171.49 \\
171.47 \\
171.10\end{array}$ & $\begin{array}{l}\mathbf{S} \\
\mathbf{S} \\
\mathbf{S} \\
\mathbf{S} \\
\mathbf{S}\end{array}$ \\
\hline & & & & & & $\begin{array}{l}02-26-87 \\
03-05-87 \\
03-12-87 \\
03-14-87 \\
03-26-87\end{array}$ & $\begin{array}{l}171.05 \\
171.64 \\
171.20 \\
171.26 \\
171.31\end{array}$ & $\begin{array}{l}\mathbf{S} \\
\mathbf{S} \\
\mathbf{S} \\
\mathbf{S} \\
\mathbf{S}\end{array}$ \\
\hline & & & & & & $\begin{array}{l}04-02-87 \\
04-09-87 \\
04-16-87 \\
04-24-87 \\
04-29-87\end{array}$ & $\begin{array}{l}173.24 \\
172.32 \\
174.55 \\
175.03 \\
176.47\end{array}$ & $\begin{array}{l}\mathbf{S} \\
\mathbf{S} \\
\mathbf{S} \\
\mathbf{S} \\
\mathbf{S}\end{array}$ \\
\hline \multirow[t]{8}{*}{$\begin{array}{l}212 \text { S20 E60 04CAD1 } \\
--/ \text { E. TOMSIK }\end{array}$} & 361417115161301 & H & 2,380 & 500 & 285 & $\begin{array}{l}02-26-86 \\
03-27-86 \\
04-09-86 \\
04-18-86 \\
05-01-86\end{array}$ & $\begin{array}{l}346.20 \\
362.57 \\
344.4 \\
344.5 \\
344.1\end{array}$ & $\begin{array}{l}\mathbf{S} \\
\mathbf{S} \\
\mathbf{T} \\
\mathbf{T} \\
\mathbf{T}\end{array}$ \\
\hline & & & & & & $\begin{array}{l}05-09-86 \\
05-15-86 \\
05-22-86 \\
05-29-86 \\
06-05-86\end{array}$ & $\begin{array}{l}344.5 \\
344.7 \\
344.8 \\
345.5 \\
344.9\end{array}$ & $\begin{array}{l}T \\
T \\
T \\
T \\
T\end{array}$ \\
\hline & & & & & & $\begin{array}{l}06-12-86 \\
06-20-86 \\
06-25-86 \\
07-03-86 \\
07-10-86\end{array}$ & $\begin{array}{l}345.1 \\
345.1 \\
344.8 \\
345.5 \\
346.9\end{array}$ & $\begin{array}{l}\mathbf{T} \\
\mathbf{T} \\
\mathbf{T} \\
\mathbf{T} \\
\mathbf{T}\end{array}$ \\
\hline & & & & & & $\begin{array}{l}07-18-86 \\
07-25-86 \\
08-01-86 \\
08-29-86 \\
09-05-86\end{array}$ & $\begin{array}{l}345.3 \\
345.3 \\
345.4 \\
347.67 \\
347.98\end{array}$ & $\begin{array}{l}\mathbf{T} \\
\mathbf{T} \\
\mathbf{T} \\
\mathbf{S} \\
\mathbf{S}\end{array}$ \\
\hline & & & & & & $\begin{array}{l}09-26-86 \\
10-03-86 \\
10-10-86 \\
10-17-86 \\
10-24-86\end{array}$ & $\begin{array}{l}348.56 \\
348.68 \\
348.49 \\
348.64 \\
349.11\end{array}$ & $\begin{array}{l}\mathbf{S} \\
\mathbf{S} \\
\mathbf{S} \\
\mathbf{S} \\
\mathbf{S}\end{array}$ \\
\hline & & & & & & $\begin{array}{l}11-05-86 \\
11-14-86 \\
11-22-86 \\
12-03-86 \\
12-09-86\end{array}$ & $\begin{array}{l}349.29 \\
349.52 \\
349.78 \\
349.80 \\
349.98\end{array}$ & $\begin{array}{l}\mathbf{S} \\
\mathbf{S} \\
\mathbf{S} \\
\mathbf{S} \\
\mathbf{S}\end{array}$ \\
\hline & & & & & & $\begin{array}{l}01-22-87 \\
01-28-87 \\
02-04-87 \\
02-12-87 \\
02-19-87\end{array}$ & $\begin{array}{l}350.30 \\
349.88 \\
350.20 \\
349.77 \\
350.03\end{array}$ & $\begin{array}{l}\mathbf{S} \\
\mathbf{S} \\
\mathbf{S} \\
\mathbf{S} \\
\mathbf{S}\end{array}$ \\
\hline & & & & & & $\begin{array}{l}02-26-87 \\
03-05-87 \\
03-12-87 \\
03-19-87 \\
03-26-87\end{array}$ & $\begin{array}{l}349.68 \\
349.54 \\
349.33 \\
349.33 \\
349.29\end{array}$ & $\begin{array}{l}\mathbf{S} \\
\mathbf{S} \\
\mathbf{S} \\
\mathbf{S} \\
\mathbf{S}\end{array}$ \\
\hline
\end{tabular}


TABLE 1.--Hater-level data for wells measured about weekly--Continued.

\begin{tabular}{|c|c|c|c|c|c|c|c|c|}
\hline \multicolumn{2}{|c|}{ Site designations } & \multirow{2}{*}{\multicolumn{2}{|c|}{$\begin{array}{c}\text { Land- } \\
\text { surface } \\
\text { al titude } \\
\text { (feet } \\
\text { above } \\
\text { Water sea } \\
\text { use level) }\end{array}$}} & \multirow[b]{2}{*}{$\begin{array}{l}\text { Depth } \\
\text { of } \\
\text { well } \\
\text { (feet) }\end{array}$} & \multirow[b]{2}{*}{$\begin{array}{l}\text { Depth to } \\
\text { shallowest } \\
\text { opening } \\
\text { (feet) }\end{array}$} & \multicolumn{3}{|c|}{ Water level } \\
\hline $\begin{array}{l}\text { Local USGS } \\
\text { identification; } \\
\text { well name or number; } \\
\text { and owner }\end{array}$ & $\begin{array}{l}\text { Standard } \\
\text { USGS } \\
\text { identification }\end{array}$ & & & & & Date & $\begin{array}{l}\text { Depth } \\
\text { (feet below } \\
\text { (and surface) }\end{array}$ & $\begin{array}{l}\text { Measure- } \\
\text { ment } \\
\text { method }\end{array}$ \\
\hline \multicolumn{2}{|c|}{212 s20 E60 04CAD1--Continued } & & & & & $\begin{array}{l}04-02-87 \\
04-09-87 \\
04-16-87 \\
04-24-87 \\
04-29-87\end{array}$ & $\begin{array}{l}348.94 \\
48.79 \\
348.73 \\
348.66 \\
348.54\end{array}$ & $\begin{array}{l}\mathbf{S} \\
\mathbf{S} \\
\mathbf{S} \\
\mathbf{S} \\
\mathbf{S}\end{array}$ \\
\hline \multirow[t]{10}{*}{$\begin{array}{l}212 \text { S20 E60 090CC1 } \\
\text { / L.MONTELLO }\end{array}$} & 361259115153901 & $\mathbf{u}$ & 2,400 & 450 & 360 & $\begin{array}{l}03-12-86 \\
03-27-86 \\
04-09-86 \\
04-18-86 \\
05-02-86\end{array}$ & $\begin{array}{l}413.94 \\
406.56 \\
401.53 \\
402.45 \\
402.3\end{array}$ & $\begin{array}{l}\mathbf{S} \\
\mathbf{S} \\
\mathbf{S} \\
\mathbf{S} \\
\mathbf{T}\end{array}$ \\
\hline & & & & & & $\begin{array}{l}05-09-86 \\
05-15-86 \\
05-22-86 \\
05-29-86 \\
06-05-86\end{array}$ & $\begin{array}{l}403.0 \\
403.1 \\
403.1 \\
402.9 \\
402.8\end{array}$ & $\begin{array}{l}T \\
T \\
T \\
T \\
T\end{array}$ \\
\hline & & & & & & $\begin{array}{l}06-12-86 \\
06-20-86 \\
06-25-86 \\
07-03-86 \\
07-10-86\end{array}$ & $\begin{array}{l}402.8 \\
403.1 \\
402.9 \\
403.6 \\
403.8\end{array}$ & $\begin{array}{l}T \\
T \\
T \\
T \\
T\end{array}$ \\
\hline & & & & & & $\begin{array}{l}07-18-86 \\
08-01-86 \\
08-08-86 \\
08-15-86 \\
08-22-86\end{array}$ & $\begin{array}{l}402.8 \\
404.54 \\
404.6 \\
405.3 \\
406.0\end{array}$ & $\begin{array}{l}T \\
T \\
T \\
T \\
T\end{array}$ \\
\hline & & & & & & $\begin{array}{l}08-29-86 \\
09-05-86 \\
09-12-86 \\
09-19-86 \\
09-26-86\end{array}$ & $\begin{array}{l}406.5 \\
406.9 \\
407.4 \\
407.5 \\
407.9\end{array}$ & $\begin{array}{l}T \\
T \\
T \\
T \\
T\end{array}$ \\
\hline & & & & & & $\begin{array}{l}10-03-86 \\
10-10-86 \\
10-17-86 \\
10-24-86 \\
10-30-86\end{array}$ & $\begin{array}{l}408.0 \\
408.0 \\
409.1 \\
409.6 \\
409.7\end{array}$ & $\begin{array}{l}T \\
T \\
T \\
T \\
T\end{array}$ \\
\hline & & & & & & $\begin{array}{l}11-07-86 \\
11-14-86 \\
11-22-86 \\
11-28-86 \\
12-03-86\end{array}$ & $\begin{array}{l}410.3 \\
410.6 \\
411.0 \\
411.2 \\
411.3\end{array}$ & $\begin{array}{l}\mathbf{T} \\
\mathbf{T} \\
\mathbf{T} \\
\mathbf{T} \\
\mathbf{T}\end{array}$ \\
\hline & & & & & & $\begin{array}{l}01-02-87 \\
01-08-87 \\
01-20-87 \\
01-28-87 \\
02-04-87\end{array}$ & $\begin{array}{l}411.9 \\
411.7 \\
411.0 \\
410.2 \\
410.0\end{array}$ & $\begin{array}{l}T \\
T \\
T \\
T \\
T\end{array}$ \\
\hline & & & & & & $\begin{array}{l}02-12-87 \\
02-20-87 \\
02-26-87 \\
03-05-87 \\
03-12-87\end{array}$ & $\begin{array}{l}409.4 \\
409.1 \\
408.8 \\
408.0 \\
407.8\end{array}$ & $\begin{array}{l}T \\
T \\
T \\
T \\
T\end{array}$ \\
\hline & & & & & & $\begin{array}{l}03-19-87 \\
04-03-87 \\
04-08-87 \\
04-16-87 \\
04-24-87 \\
04-29-87\end{array}$ & $\begin{array}{l}407.4 \\
406.2 \\
406.4 \\
406.2 \\
406.0 \\
405.6\end{array}$ & $\begin{array}{l}T \\
T \\
T \\
T \\
T \\
T\end{array}$ \\
\hline
\end{tabular}


TABLE 1.--Water-level data for wells measured about weekly--Cont inued.

\begin{tabular}{|c|c|c|c|c|c|c|c|c|}
\hline \multicolumn{2}{|c|}{ Site designations } & \multirow{2}{*}{\multicolumn{2}{|c|}{$\begin{array}{cc}\text { Land- } \\
\text { surface } \\
\text { al titude } \\
\text { (feet } \\
\text { above } \\
\text { Water sea } \\
\text { use level) }\end{array}$}} & \multirow[b]{2}{*}{$\begin{array}{l}\text { Depth } \\
\text { of } \\
\text { well } \\
\text { (feet) }\end{array}$} & \multirow[b]{2}{*}{$\begin{array}{l}\text { Depth to } \\
\text { shallowest } \\
\text { opening } \\
\text { (feet) }\end{array}$} & \multicolumn{3}{|c|}{ Water level } \\
\hline $\begin{array}{l}\text { Local USGS } \\
\text { identification; } \\
\text { well name or number; } \\
\text { and owner }\end{array}$ & $\begin{array}{l}\text { Stanclard } \\
\text { USGS } \\
\text { identification }\end{array}$ & & & & & Date & $\begin{array}{l}\text { Depth } \\
\text { (feet below } \\
\text { land surface) }\end{array}$ & $\begin{array}{l}\text { Measure } \\
\text { ment } \\
\text { method }\end{array}$ \\
\hline $\begin{array}{l}212 \text { S20 E60 120BB1 } \\
\text { ELSTNER ESTATES / } \\
\text { CITY N.L.V. }\end{array}$ & 361303115124501 & $\cdots$ & 2,252 & 800 & 160 & $\begin{array}{l}03-18-86 \\
05-02-86 \\
05-15-86 \\
05-22-86 \\
05-29-86 \\
06-12-86\end{array}$ & $\begin{array}{l}269.78 \\
262.17 \\
263.77 \\
262.68 \\
261.71 \\
262.19\end{array}$ & $\begin{array}{l}\mathbf{S} \\
\mathbf{S} \\
\mathbf{S} \\
\mathbf{S} \\
\mathbf{S} \\
\mathbf{S}\end{array}$ \\
\hline \multirow[t]{10}{*}{$\begin{array}{ll}212 & \text { S20 E60 120CB1 } \\
\# 46 / \text { LWWD } & \end{array}$} & 361254115124701 & $\cdots$ & 2,252 & 355 & 100 & $\begin{array}{l}03-12-86 \\
03-27-86 \\
04-09-86 \\
04-18-86 \\
05-01-86\end{array}$ & $\begin{array}{l}99.83 \\
99.45 \\
99.33 \\
99.41 \\
99.45\end{array}$ & $\begin{array}{l}\mathbf{S} \\
\mathbf{S} \\
\mathbf{S} \\
\mathbf{S} \\
\mathbf{S}\end{array}$ \\
\hline & & & & & & $\begin{array}{l}05-09-86 \\
05-15-86 \\
05-22-86 \\
05-29-86 \\
06-05-86\end{array}$ & $\begin{array}{l}101.38 \\
102.07 \\
99.37 \\
99.27 \\
99.35\end{array}$ & $\begin{array}{l}\mathbf{S} \\
\mathbf{S} \\
\mathbf{S} \\
\mathbf{S} \\
\mathbf{S}\end{array}$ \\
\hline & & & & & & $\begin{array}{l}06-12-86 \\
06-20-86 \\
06-25-86 \\
07-03-86 \\
07-10-86\end{array}$ & $\begin{array}{l}99.60 \\
99.45 \\
98.7 \\
99.39 \\
99.69\end{array}$ & $\begin{array}{l}\mathbf{S} \\
\mathbf{S} \\
\mathbf{T} \\
\mathbf{S} \\
\mathbf{S}\end{array}$ \\
\hline & & & & & & $\begin{array}{l}07-18-86 \\
07-25-86 \\
08-01-86 \\
08-08-86 \\
08-15-86\end{array}$ & $\begin{array}{l}99.38 \\
99.42 \\
99.38 \\
99.33 \\
99.36\end{array}$ & $\begin{array}{l}\mathbf{S} \\
\mathbf{S} \\
\mathbf{S} \\
\mathbf{S} \\
\mathbf{S}\end{array}$ \\
\hline & & & & & & $\begin{array}{l}08-22-86 \\
08-29-86 \\
09-05-86 \\
09-12-86 \\
09-19-86\end{array}$ & $\begin{array}{l}99.48 \\
99.46 \\
99.51 \\
99.34 \\
99.24\end{array}$ & $\begin{array}{l}\mathbf{S} \\
\mathbf{S} \\
\mathbf{S} \\
\mathbf{S} \\
\mathbf{S}\end{array}$ \\
\hline & & & & & & $\begin{array}{l}09-26-86 \\
10-02-86 \\
10-10-86 \\
10-17-86 \\
10-24-86\end{array}$ & $\begin{array}{l}99.29 \\
99.34 \\
99.30 \\
99.05 \\
99.39\end{array}$ & $\begin{array}{l}\mathbf{S} \\
\mathbf{S} \\
\mathbf{S} \\
\mathbf{S} \\
\mathbf{S}\end{array}$ \\
\hline & & & & & & $\begin{array}{l}10-30-86 \\
11-05-86 \\
11-14-86 \\
11-22-86 \\
11-28-86\end{array}$ & $\begin{array}{l}99.65 \\
99.01 \\
99.54 \\
99.00 \\
99.11\end{array}$ & $\begin{array}{l}\mathbf{S} \\
\mathbf{S} \\
\mathbf{S} \\
\mathbf{S} \\
\mathbf{S}\end{array}$ \\
\hline & & & & & & $\begin{array}{l}12-03-86 \\
12-09-86 \\
12-19-86 \\
12-23-86 \\
01-02-87\end{array}$ & $\begin{array}{l}99.01 \\
99.44 \\
99.36 \\
99.45 \\
99.32\end{array}$ & $\begin{array}{l}\mathbf{S} \\
\mathbf{S} \\
\mathbf{S} \\
\mathbf{S} \\
\mathbf{S}\end{array}$ \\
\hline & & & & & & $\begin{array}{l}01-08-87 \\
01-20-87 \\
01-22-87 \\
01-28-87 \\
02-04-87\end{array}$ & $\begin{array}{l}99.39 \\
99.43 \\
99.38 \\
99.49 \\
99.19\end{array}$ & $\begin{array}{l}\mathbf{S} \\
\mathbf{S} \\
\mathbf{S} \\
\mathbf{S} \\
\mathbf{S}\end{array}$ \\
\hline & & & & & & $\begin{array}{l}02-12-87 \\
02-19-87 \\
02-26-87 \\
03-05-87 \\
03-12-87\end{array}$ & $\begin{array}{l}99.03 \\
99.47 \\
99.54 \\
99.57 \\
99.50\end{array}$ & $\begin{array}{l}\mathbf{S} \\
\mathbf{S} \\
\mathbf{S} \\
\mathbf{S} \\
\mathbf{S}\end{array}$ \\
\hline
\end{tabular}


TABLE 1.--Water-level data for wells measured about weekly--Cont inued.

\begin{tabular}{|c|c|c|c|c|c|c|c|c|}
\hline \multicolumn{2}{|c|}{ Site designations } & \multirow[b]{2}{*}{$\begin{array}{c}\text { Water } \\
\text { use }\end{array}$} & \multirow{2}{*}{$\begin{array}{l}\text { Land- } \\
\text { surface } \\
\text { altitude } \\
\text { (feet } \\
\text { above } \\
\text { sea } \\
\text { level) }\end{array}$} & \multirow[b]{2}{*}{$\begin{array}{c}\text { Depth } \\
\text { of } \\
\text { well } \\
\text { (feet) }\end{array}$} & \multirow[b]{2}{*}{$\begin{array}{l}\text { Depth to } \\
\text { shall lowest } \\
\text { opening } \\
\text { (feet) }\end{array}$} & \multicolumn{3}{|c|}{ Water level } \\
\hline $\begin{array}{l}\text { Local USGS } \\
\text { identification; } \\
\text { well name or number; } \\
\text { and owner }\end{array}$ & $\begin{array}{l}\text { Standard } \\
\text { USGS } \\
\text { identification }\end{array}$ & & & & & Date & $\begin{array}{l}\text { Depth } \\
\text { (feet below } \\
\text { land surface) }\end{array}$ & $\begin{array}{l}\text { Measure- } \\
\text { ment } \\
\text { method }\end{array}$ \\
\hline \multirow{2}{*}{\multicolumn{2}{|c|}{212 S20 E60 12DCB1--Continued }} & & & & & $\begin{array}{l}03-20-87 \\
03-26-87 \\
04-02-87 \\
04-09-87 \\
04-16-87\end{array}$ & $\begin{array}{l}99.53 \\
99.38 \\
99.30 \\
99.30 \\
99.40\end{array}$ & $\begin{array}{l}\mathbf{S} \\
\mathbf{S} \\
\mathbf{S} \\
\mathbf{S} \\
\mathbf{S}\end{array}$ \\
\hline & & & & & & $\begin{array}{l}04-24-87 \\
04-29-87\end{array}$ & $\begin{array}{l}99.41 \\
99.33\end{array}$ & $\begin{array}{l}\mathbf{S} \\
\mathbf{S}\end{array}$ \\
\hline \multirow[t]{8}{*}{$\begin{array}{l}212 \text { S20 E60 34CCCC2 } \\
\# 21 \text { / LVVD }\end{array}$} & 360935115153201 & $\mathbf{P}$ & 2,506 & 1000 & 465 & $\begin{array}{l}03-27-86 \\
03-27-86 \\
04-09-86 \\
04-22-86 \\
05-01-86\end{array}$ & $\begin{array}{l}532.00 \\
532.0 \\
530.9 \\
530.6 \\
531.4\end{array}$ & $\begin{array}{l}T \\
T \\
T \\
T \\
T\end{array}$ \\
\hline & & & & & & $\begin{array}{l}05-12-86 \\
05-19-86 \\
05-23-86 \\
05-29-86 \\
06-06-86\end{array}$ & $\begin{array}{l}530.7 \\
530.8 \\
530.7 \\
530.6 \\
530.8\end{array}$ & $\begin{array}{l}T \\
T \\
T \\
T \\
T\end{array}$ \\
\hline & & & & & & $\begin{array}{l}06-12-86 \\
06-20-86 \\
06-25-86 \\
07-03-86 \\
07-10-86\end{array}$ & $\begin{array}{l}530.6 \\
530.8 \\
531.5 \\
532.1 \\
532.4\end{array}$ & $\begin{array}{l}T \\
\mathbf{T} \\
\mathbf{T} \\
\mathbf{T} \\
\mathbf{T}\end{array}$ \\
\hline & & & & & & $\begin{array}{l}07-17-86 \\
07-25-86 \\
08-22-86 \\
08-29-86 \\
09-05-86\end{array}$ & $\begin{array}{l}532.5 \\
532.8 \\
536.4 \\
536.4 \\
536.7\end{array}$ & $\begin{array}{l}T \\
T \\
T \\
T \\
T\end{array}$ \\
\hline & & & & & & $\begin{array}{l}09-12-86 \\
09-19-86 \\
09-26-86 \\
10-03-86 \\
10-10-86\end{array}$ & $\begin{array}{l}536.8 \\
536.9 \\
537.2 \\
537.8 \\
537.7\end{array}$ & $\begin{array}{l}T \\
T \\
T \\
T \\
T\end{array}$ \\
\hline & & & & & & $\begin{array}{l}10-23-86 \\
10-31-86 \\
11-07-86 \\
11-14-86 \\
11-21-86\end{array}$ & $\begin{array}{l}538.0 \\
538.2 \\
538.5 \\
538.6 \\
539.3\end{array}$ & $\begin{array}{l}T \\
T \\
T \\
T \\
T\end{array}$ \\
\hline & & & & & & $\begin{array}{l}11-28-86 \\
12-05-86 \\
12-19-86 \\
12-31-86 \\
01-08-87\end{array}$ & $\begin{array}{l}539.60 \\
539.4 \\
539.3 \\
539.0 \\
539.1\end{array}$ & $\begin{array}{l}\mathbf{T} \\
\mathbf{T} \\
\mathbf{T} \\
\mathbf{T} \\
\mathbf{T}\end{array}$ \\
\hline & & & & & & $\begin{array}{l}01-20-87 \\
02-06-87 \\
02-27-87 \\
03-06-87 \\
04-22-87 \\
04-30-87\end{array}$ & $\begin{array}{l}538.7 \\
538.1 \\
538.2 \\
536.0 \\
533.7 \\
533.3\end{array}$ & $\begin{array}{l}T \\
T \\
T \\
T \\
T \\
T\end{array}$ \\
\hline \multirow[t]{2}{*}{$\begin{array}{c}212 \text { S20 E61 02DBB1 } \\
\text { HARTWELL \& } \\
\text { LOWE CO. }\end{array}$} & 361419115072201 & $\mathbf{U}$ & 1,900 & 785 & 90 & $\begin{array}{l}02-19-86 \\
03-19-86 \\
03-27-86 \\
04-09-86 \\
04-18-86\end{array}$ & $\begin{array}{l}43.54 \\
42.60 \\
43.37 \\
41.86 \\
41.63\end{array}$ & $\begin{array}{l}\mathbf{S} \\
\mathbf{S} \\
\mathbf{S} \\
\mathbf{S} \\
\mathbf{S}\end{array}$ \\
\hline & & & & & & $\begin{array}{l}05-01-86 \\
05-09-86 \\
05-15-86 \\
05-22-86 \\
05-29-86\end{array}$ & $\begin{array}{l}41.15 \\
41.32 \\
41.94 \\
42.81 \\
42.42\end{array}$ & $\begin{array}{l}\mathbf{S} \\
\mathbf{S} \\
\mathbf{S} \\
\mathbf{S} \\
\mathbf{S}\end{array}$ \\
\hline
\end{tabular}


TABLE 1.--Water-level data for wells measured about weekly--Cont inued.

\begin{tabular}{|c|c|c|c|c|c|c|c|c|}
\hline \multicolumn{2}{|c|}{ Site designations } & \multirow{2}{*}{\multicolumn{2}{|c|}{$\begin{array}{c}\text { Land- } \\
\text { surface } \\
\text { altitude } \\
\text { (feet } \\
\text { above }\end{array}$}} & \multirow[b]{2}{*}{$\begin{array}{c}\text { Depth } \\
\text { of } \\
\text { well } \\
\text { (feet) }\end{array}$} & \multirow[b]{2}{*}{$\begin{array}{l}\text { Depth to } \\
\text { shall lowest } \\
\text { opening } \\
\text { (feet) }\end{array}$} & \multicolumn{3}{|c|}{ Water level } \\
\hline $\begin{array}{l}\text { Local USGS } \\
\text { identification; } \\
\text { well name or number; } \\
\text { and owner }\end{array}$ & $\begin{array}{c}\text { Standard } \\
\text { USGS } \\
\text { identification }\end{array}$ & & & & & Date & $\begin{array}{l}\text { Depth } \\
\text { (feet below } \\
\text { land surface) }\end{array}$ & $\begin{array}{l}\text { Measure- } \\
\text { ment } \\
\text { method }\end{array}$ \\
\hline \multirow{3}{*}{\multicolumn{2}{|c|}{212 S20 E61 020BB1--Cont inued }} & & & & & $\begin{array}{l}06-05-86 \\
06-12-86 \\
06-20-86 \\
06-25-86 \\
07-03-86\end{array}$ & $\begin{array}{l}44.72 \\
46.89 \\
49.58 \\
51.03 \\
52.27\end{array}$ & $\begin{array}{l}\mathbf{S} \\
\mathbf{S} \\
\mathbf{S} \\
\mathbf{S} \\
\mathbf{S}\end{array}$ \\
\hline & & & & & & $\begin{array}{l}07-10-86 \\
07-18-86 \\
07-25-86 \\
08-01-86 \\
08-07-86\end{array}$ & $\begin{array}{l}53.30 \\
55.27 \\
56.07 \\
57.60 \\
58.39\end{array}$ & $\begin{array}{l}\mathbf{S} \\
\mathbf{S} \\
\mathbf{S} \\
\mathbf{S} \\
\mathbf{S}\end{array}$ \\
\hline & & & & & & $\begin{array}{l}02-26-87 \\
04-03-87 \\
04-09-87 \\
04-16-87 \\
04-22-87 \\
04-29-87\end{array}$ & $\begin{array}{l}51.56 \\
42.19 \\
42.05 \\
42.31 \\
41.88 \\
41.76\end{array}$ & $\begin{array}{l}\mathbf{S} \\
\mathbf{S} \\
\mathbf{S} \\
\mathbf{S} \\
\mathbf{S} \\
\mathbf{S}\end{array}$ \\
\hline \multirow[t]{8}{*}{212 S20 E61 04BDC1 } & 361426115095001 & $\mathbf{u}$ & 2,103 & 270 & $\cdots$ & $\begin{array}{l}02-19-86 \\
03-27-86 \\
04-09-86 \\
04-18-86 \\
05-01-86\end{array}$ & $\begin{array}{l}43.54 \\
94.42 \\
90.97 \\
90.97 \\
89.08\end{array}$ & $\begin{array}{l}\mathbf{S} \\
\mathbf{S} \\
\mathbf{S} \\
\mathbf{S} \\
\mathbf{S}\end{array}$ \\
\hline & & & & & & $\begin{array}{l}05-09-86 \\
05-15-86 \\
05-22-86 \\
05-29-86 \\
06-05-86\end{array}$ & $\begin{array}{l}91.02 \\
91.17 \\
91.19 \\
91.22 \\
91.25\end{array}$ & $\begin{array}{l}\mathbf{S} \\
\mathbf{S} \\
\mathbf{S} \\
\mathbf{S} \\
\mathbf{S}\end{array}$ \\
\hline & & & & & & $\begin{array}{l}06-12-86 \\
06-20-86 \\
06-25-86 \\
07-03-86 \\
07-10-86\end{array}$ & $\begin{array}{l}91.24 \\
91.42 \\
91.5 \\
91.50 \\
91.76\end{array}$ & $\begin{array}{l}\mathbf{S} \\
\mathbf{S} \\
\mathbf{T} \\
\mathbf{S} \\
\mathbf{S}\end{array}$ \\
\hline & & & & & & $\begin{array}{l}07-18-86 \\
07-25-86 \\
08-01-86 \\
08-07-86 \\
08-14-86\end{array}$ & $\begin{array}{l}91.65 \\
92.71 \\
91.77 \\
91.81 \\
91.84\end{array}$ & $\begin{array}{l}\mathbf{S} \\
\mathbf{S} \\
\mathbf{S} \\
\mathbf{S} \\
\mathbf{S}\end{array}$ \\
\hline & & & & & & $\begin{array}{l}08-22-86 \\
08-29-86 \\
09-05-86 \\
09-12-86 \\
09-19-86\end{array}$ & $\begin{array}{l}91.98 \\
92.00 \\
92.06 \\
92.16 \\
92.14\end{array}$ & $\begin{array}{l}\mathbf{S} \\
\mathbf{S} \\
\mathbf{S} \\
\mathbf{S} \\
\mathbf{S}\end{array}$ \\
\hline & & & & & & $\begin{array}{l}09-26-86 \\
10-03-86 \\
10-10-86 \\
10-17-86 \\
10-23-86\end{array}$ & $\begin{array}{l}92.21 \\
92.22 \\
92.21 \\
92.20 \\
92.24\end{array}$ & $\begin{array}{l}\mathbf{S} \\
\mathbf{S} \\
\mathbf{S} \\
\mathbf{S} \\
\mathbf{S}\end{array}$ \\
\hline & & & & & & $\begin{array}{l}10-30-86 \\
11-05-86 \\
11-14-86 \\
11-21-86 \\
11-28-86\end{array}$ & $\begin{array}{l}92.19 \\
92.25 \\
92.26 \\
92.25 \\
92.24\end{array}$ & $\begin{array}{l}\mathbf{S} \\
\mathbf{S} \\
\mathbf{S} \\
\mathbf{S} \\
\mathbf{S}\end{array}$ \\
\hline & & & & & & $\begin{array}{l}12-03-86 \\
12-09-86 \\
12-19-86 \\
12-31-86 \\
01-08-87\end{array}$ & $\begin{array}{l}92.25 \\
92.30 \\
92.23 \\
92.27 \\
92.26\end{array}$ & $\begin{array}{l}\mathbf{S} \\
\mathbf{S} \\
\mathbf{S} \\
\mathbf{S} \\
\mathbf{S}\end{array}$ \\
\hline
\end{tabular}


TABLE 1.--Hater-level data for wells measured about weekly--Cont inued.

\begin{tabular}{|c|c|c|c|c|c|c|c|c|}
\hline \multicolumn{2}{|c|}{ site designations } & \multirow{2}{*}{\multicolumn{2}{|c|}{$\begin{array}{cc}\text { Land- } \\
\text { surface } \\
\text { altitude } \\
\text { (feet } \\
\text { above } \\
\text { Water sea } \\
\text { use level) }\end{array}$}} & \multirow[b]{2}{*}{$\begin{array}{l}\text { Depth } \\
\text { of } \\
\text { well } \\
\text { (feet) }\end{array}$} & \multirow[b]{2}{*}{$\begin{array}{l}\text { Depth to } \\
\text { shallowest } \\
\text { opening } \\
\text { (feet) }\end{array}$} & \multicolumn{3}{|c|}{ Water level } \\
\hline $\begin{array}{l}\text { Local USGS } \\
\text { identification; } \\
\text { well name or number; } \\
\text { and owner }\end{array}$ & $\begin{array}{c}\text { Standard } \\
\text { USGS } \\
\text { identification }\end{array}$ & & & & & Date & $\begin{array}{l}\text { Depth } \\
\text { (feet below } \\
\text { land surface) }\end{array}$ & $\begin{array}{l}\text { Measure- } \\
\text { ment } \\
\text { method }\end{array}$ \\
\hline \multirow{3}{*}{\multicolumn{2}{|c|}{212 S20 E61 0480C1--Continued }} & & & & & $\begin{array}{l}01-20-87 \\
01-28-87 \\
02-04-87 \\
02-12-87 \\
02-19-87\end{array}$ & $\begin{array}{l}92.24 \\
92.24 \\
92.28 \\
92.27 \\
92.29\end{array}$ & $\begin{array}{l}\mathbf{S} \\
\mathbf{S} \\
\mathbf{S} \\
\mathbf{S} \\
\mathbf{S}\end{array}$ \\
\hline & & & & & & $\begin{array}{l}02-26-87 \\
03-05-87 \\
03-12-87 \\
03-20-87 \\
03-26-87\end{array}$ & $\begin{array}{l}92.33 \\
92.31 \\
92.30 \\
92.33 \\
92.34\end{array}$ & $\begin{array}{l}S \\
S \\
S \\
S \\
S\end{array}$ \\
\hline & & & & & & $\begin{array}{l}04-03-87 \\
04-09-87 \\
04-16-87 \\
04-22-87 \\
04-29-87\end{array}$ & $\begin{array}{l}92.26 \\
92.37 \\
92.39 \\
92.40 \\
92.43\end{array}$ & $\begin{array}{l}\mathbf{S} \\
\mathbf{S} \\
\mathbf{S} \\
\mathbf{S} \\
\mathbf{S}\end{array}$ \\
\hline \multirow[t]{8}{*}{$\begin{array}{l}212 \text { S20 E61 13ABDB1 } \\
\text { DIANA TERRACE / } \\
\text { CITY N.L.V. }\end{array}$} & 361232115061001 & $\mathbf{P}$ & 1,857 & 1,230 & 102 & $\begin{array}{l}03-14-86 \\
03-24-86 \\
04-09-86 \\
04-22-86 \\
05-01-86\end{array}$ & $\begin{array}{l}53.19 \\
56.30 \\
52.95 \\
52.85 \\
52.79\end{array}$ & $\begin{array}{l}\mathbf{S} \\
\mathbf{S} \\
\mathbf{S} \\
\mathbf{S} \\
\mathbf{S}\end{array}$ \\
\hline & & & & & & $\begin{array}{l}05-09-86 \\
05-19-86 \\
05-23-86 \\
05-29-86 \\
06-05-86\end{array}$ & $\begin{array}{l}52.79 \\
52.68 \\
52.63 \\
53.45 \\
57.05\end{array}$ & $\begin{array}{l}\mathbf{S} \\
\mathbf{S} \\
\mathbf{S} \\
\mathbf{S} \\
\mathbf{S}\end{array}$ \\
\hline & & & & & & $\begin{array}{l}06-12-86 \\
06-20-86 \\
06-25-86 \\
07-03-86 \\
07-10-86\end{array}$ & $\begin{array}{l}59.16 \\
61.04 \\
61.94 \\
62.57 \\
63.55\end{array}$ & $\begin{array}{l}\mathbf{S} \\
\mathbf{S} \\
\mathbf{S} \\
\mathbf{S} \\
\mathbf{S}\end{array}$ \\
\hline & & & & & & $\begin{array}{l}07-18-86 \\
07-25-86 \\
08-01-86 \\
08-08-86 \\
08-15-86\end{array}$ & $\begin{array}{l}64.44 \\
64.69 \\
65.09 \\
65.45 \\
65.63\end{array}$ & $\begin{array}{l}\mathbf{S} \\
\mathbf{S} \\
\mathbf{S} \\
\mathbf{S} \\
\mathbf{S}\end{array}$ \\
\hline & & & & & & $\begin{array}{l}08-22-86 \\
08-29-86 \\
09-05-86 \\
09-12-86 \\
09-19-86\end{array}$ & $\begin{array}{l}66.07 \\
66.29 \\
66.51 \\
67.16 \\
67.29\end{array}$ & $\begin{array}{l}S \\
S \\
S \\
S \\
S\end{array}$ \\
\hline & & & & & & $\begin{array}{l}09-26-86 \\
10-03-86 \\
10-10-86 \\
10-17-86 \\
10-24-86\end{array}$ & $\begin{array}{l}60.58 \\
59.11 \\
58.44 \\
57.59 \\
56.94\end{array}$ & $\begin{array}{l}\mathbf{S} \\
\mathbf{S} \\
\mathbf{S} \\
\mathbf{S} \\
\mathbf{S}\end{array}$ \\
\hline & & & & & & $\begin{array}{l}10-31-86 \\
11-05-86 \\
11-14-86 \\
11-22-86 \\
11-28-86\end{array}$ & $\begin{array}{l}56.37 \\
56.09 \\
55.65 \\
55.29 \\
55.07\end{array}$ & $\begin{array}{l}\mathbf{S} \\
\mathbf{S} \\
\mathbf{S} \\
\mathbf{S} \\
\mathbf{S}\end{array}$ \\
\hline & & & & & & $\begin{array}{l}12-03-86 \\
12-09-86 \\
12-19-86 \\
12-23-86\end{array}$ & $\begin{array}{l}54.90 \\
54.74 \\
54.43 \\
54.30\end{array}$ & $\begin{array}{l}\mathbf{S} \\
\mathbf{S} \\
\mathbf{S} \\
\mathbf{S}\end{array}$ \\
\hline
\end{tabular}


TABLE 1.--Hater-level data for wells measured about weekly--Cont inued.

\begin{tabular}{|c|c|c|c|c|c|c|c|c|}
\hline \multicolumn{2}{|c|}{ site designations } & \multirow{2}{*}{\multicolumn{2}{|c|}{$\begin{array}{c}\text { Land- } \\
\text { surface } \\
\text { altitude } \\
\text { (feet } \\
\text { above } \\
\text { Water sea } \\
\text { use level) }\end{array}$}} & \multirow[b]{2}{*}{$\begin{array}{l}\text { Depth } \\
\text { of } \\
\text { well } \\
\text { (feet) }\end{array}$} & \multirow[b]{2}{*}{$\begin{array}{l}\text { Depth to } \\
\text { shallowest } \\
\text { opening } \\
\text { (feet) }\end{array}$} & \multicolumn{3}{|c|}{ Water level } \\
\hline $\begin{array}{l}\text { LOcal USGS } \\
\text { identification; } \\
\text { well name or number; } \\
\text { and owner }\end{array}$ & $\begin{array}{l}\text { Standard } \\
\text { USGS } \\
\text { identification }\end{array}$ & & & & & Date & $\begin{array}{l}\text { Depth } \\
\text { (feet below } \\
\text { land surface) }\end{array}$ & $\begin{array}{l}\text { Measure- } \\
\text { ment } \\
\text { method }\end{array}$ \\
\hline \multirow[t]{11}{*}{$\begin{array}{l}212 \text { S20 E61 } 20 C D C 1 \\
\# 3 A \text { / LWWD }\end{array}$} & 361120115105301 & $\mathbf{P}$ & 2,108 & 925 & 300 & $\begin{array}{l}03-13-86 \\
03-27-86 \\
04-08-86 \\
04-18-86 \\
05-01-86\end{array}$ & $\begin{array}{l}149.08 \\
160.33 \\
159.34 \\
149.94 \\
149.76\end{array}$ & $\begin{array}{l}\mathbf{S} \\
\mathbf{S} \\
\mathbf{S} \\
\mathbf{S} \\
\mathbf{S}\end{array}$ \\
\hline & & & & & & $\begin{array}{l}05-09-86 \\
05-19-86 \\
05-22-86 \\
05-29-86 \\
06-05-86\end{array}$ & $\begin{array}{l}153.01 \\
152.62 \\
152.90 \\
154.04 \\
157.44\end{array}$ & $\begin{array}{l}\mathbf{S} \\
\mathbf{S} \\
\mathbf{S} \\
\mathbf{S} \\
\mathbf{S}\end{array}$ \\
\hline & & & & & & $\begin{array}{l}06-12-86 \\
06-19-86 \\
06-23-86 \\
07-03-86 \\
07-10-86\end{array}$ & $\begin{array}{l}159.41 \\
162.65 \\
162.67 \\
163.59 \\
166.01\end{array}$ & $\begin{array}{l}\mathbf{S} \\
\mathbf{S} \\
\mathbf{S} \\
\mathbf{S} \\
\mathbf{S}\end{array}$ \\
\hline & & & & & & $\begin{array}{l}07-18-86 \\
07-25-86 \\
08-01-86 \\
08-08-86 \\
08-15-86\end{array}$ & $\begin{array}{l}167.91 \\
168.98 \\
170.00 \\
171.69 \\
172.02\end{array}$ & $\begin{array}{l}\mathbf{S} \\
\mathbf{S} \\
\mathbf{S} \\
\mathbf{S} \\
\mathbf{S}\end{array}$ \\
\hline & & & & & & $\begin{array}{l}08-22-86 \\
08-29-86 \\
09-05-86 \\
09-12-86 \\
09-19-86\end{array}$ & $\begin{array}{l}173.18 \\
173.62 \\
174.25 \\
173.91 \\
173.95\end{array}$ & $\begin{array}{l}\mathbf{S} \\
\mathbf{S} \\
\mathbf{S} \\
\mathbf{S} \\
\mathbf{S}\end{array}$ \\
\hline & & & & & & $\begin{array}{l}10-03-86 \\
10-10-86 \\
10-17-86 \\
10-23-86 \\
10-30-86\end{array}$ & $\begin{array}{l}173.3 \\
172.72 \\
172.37 \\
170.88 \\
169.97\end{array}$ & $\begin{array}{l}T \\
\mathbf{S} \\
\mathbf{S} \\
\mathbf{S} \\
\mathbf{S}\end{array}$ \\
\hline & & & & & & $\begin{array}{l}11-05-86 \\
11-22-86 \\
12-03-86 \\
12-09-86 \\
12-15-86\end{array}$ & $\begin{array}{l}168.76 \\
164.94 \\
163.59 \\
162.23 \\
160.85\end{array}$ & $\begin{array}{l}\mathbf{S} \\
\mathbf{S} \\
\mathbf{S} \\
\mathbf{S} \\
\mathbf{S}\end{array}$ \\
\hline & & & & & & $\begin{array}{l}12-19-86 \\
12-23-86 \\
12-31-86 \\
01-08-87 \\
01-20-87\end{array}$ & $\begin{array}{l}160.64 \\
160.48 \\
159.45 \\
156.71 \\
154.88\end{array}$ & $\begin{array}{l}\mathbf{S} \\
\mathbf{S} \\
\mathbf{S} \\
\mathbf{S} \\
\mathbf{S}\end{array}$ \\
\hline & & & & & & $\begin{array}{l}01-28-87 \\
02-04-87 \\
02-12-87 \\
02-19-87 \\
02-27-87\end{array}$ & $\begin{array}{l}153.91 \\
153.25 \\
152.48 \\
151.74 \\
150.68\end{array}$ & $\begin{array}{l}\mathbf{S} \\
\mathbf{S} \\
\mathbf{S} \\
\mathbf{S} \\
\mathbf{S}\end{array}$ \\
\hline & & & & & & $\begin{array}{l}03-06-87 \\
03-12-87 \\
03-26-87 \\
04-02-87 \\
04-09-87\end{array}$ & $\begin{array}{l}151.37 \\
150.87 \\
150.10 \\
148.93 \\
149.09\end{array}$ & $\begin{array}{l}\mathbf{S} \\
\mathbf{S} \\
\mathbf{S} \\
\mathbf{S} \\
\mathbf{S}\end{array}$ \\
\hline & & & & & & $\begin{array}{l}04-16-87 \\
04-22-87 \\
04-29-87\end{array}$ & $\begin{array}{l}150.01 \\
151.23 \\
151.35\end{array}$ & $\begin{array}{l}\mathbf{S} \\
\mathbf{S} \\
\mathbf{S}\end{array}$ \\
\hline
\end{tabular}


TABLE 1.--Water-level data for wells measured about weekly--Continued.

\begin{tabular}{|c|c|c|c|c|c|c|c|c|}
\hline \multicolumn{2}{|c|}{ Site designations } & \multirow{2}{*}{\multicolumn{2}{|c|}{$\begin{array}{c}\text { Land- } \\
\text { surface } \\
\text { al titude } \\
\text { (feet } \\
\text { above } \\
\text { Water sea } \\
\text { use level) }\end{array}$}} & \multirow[b]{2}{*}{$\begin{array}{l}\text { Depth } \\
\text { of } \\
\text { well } \\
\text { (feet) }\end{array}$} & \multirow[b]{2}{*}{$\begin{array}{l}\text { Depth to } \\
\text { shallowest } \\
\text { opening } \\
\text { (feet) }\end{array}$} & \multicolumn{3}{|c|}{ Water level } \\
\hline $\begin{array}{l}\text { Local USGS } \\
\text { identification; } \\
\text { well name or number; } \\
\text { and owner }\end{array}$ & $\begin{array}{c}\text { Standard } \\
\text { UStiS } \\
\text { identification }\end{array}$ & & & & & Date & $\begin{array}{l}\text { Depth } \\
\text { (feet below } \\
\text { land surface) }\end{array}$ & $\begin{array}{l}\text { Measure- } \\
\text { ment } \\
\text { method }\end{array}$ \\
\hline \multirow[t]{10}{*}{$\begin{array}{c}212 \text { S20 E61 21BAA1 } \\
\text { BOOKER / L.V. UNION } \\
\text { SCHOOL } \\
\text { DIST. }\end{array}$} & 361147115094001 & $\mathbf{P}$ & 2,064 & 397 & 200 & $\begin{array}{l}02-26-86 \\
03-27-86 \\
04-09-86 \\
04-22-86 \\
05-01-86\end{array}$ & $\begin{array}{l}75.05 \\
74.32 \\
73.89 \\
73.57 \\
73.50\end{array}$ & $\begin{array}{l}\mathbf{S} \\
\mathbf{S} \\
\mathbf{S} \\
\mathbf{S} \\
\mathbf{S}\end{array}$ \\
\hline & & & & & & $\begin{array}{l}05-09-86 \\
05-19-86 \\
05-23-86 \\
05-29-86 \\
06-05-86\end{array}$ & $\begin{array}{l}74.10 \\
73.42 \\
73.50 \\
73.59 \\
74.12\end{array}$ & $\begin{array}{l}\mathbf{S} \\
\mathbf{S} \\
\mathbf{S} \\
\mathbf{S} \\
\mathbf{S}\end{array}$ \\
\hline & & & & & & $\begin{array}{l}06-12-86 \\
06-20-86 \\
06-25-86 \\
07-03-86 \\
07-10-86\end{array}$ & $\begin{array}{l}74.80 \\
75.67 \\
76.19 \\
76.48 \\
77.36\end{array}$ & $\begin{array}{l}\mathbf{S} \\
\mathbf{S} \\
\mathbf{S} \\
\mathbf{S} \\
\mathbf{S}\end{array}$ \\
\hline & & & & & & $\begin{array}{l}08-01-86 \\
08-08-86 \\
08-22-86 \\
08-29-86 \\
09-05-86\end{array}$ & $\begin{array}{l}78.38 \\
79.17 \\
79.89 \\
80.10 \\
80.29\end{array}$ & $\begin{array}{l}\mathbf{S} \\
\mathbf{S} \\
\mathbf{S} \\
\mathbf{S} \\
\mathbf{S}\end{array}$ \\
\hline & & & & & & $\begin{array}{l}09-12-86 \\
09-19-86 \\
09-26-86 \\
10-03-86 \\
10-07-86\end{array}$ & $\begin{array}{l}80.62 \\
80.71 \\
80.91 \\
80.90 \\
80.76\end{array}$ & $\begin{array}{l}\mathbf{S} \\
\mathbf{S} \\
\mathbf{S} \\
\mathbf{S} \\
\mathbf{S}\end{array}$ \\
\hline & & & & & & $\begin{array}{l}10-17-86 \\
10-23-86 \\
10-30-86 \\
11-05-86 \\
11-14-86\end{array}$ & $\begin{array}{l}80.49 \\
80.23 \\
79.86 \\
79.62 \\
79.30\end{array}$ & $\begin{array}{l}\mathbf{S} \\
\mathbf{S} \\
\mathbf{S} \\
\mathbf{S} \\
\mathbf{S}\end{array}$ \\
\hline & & & & & & $\begin{array}{l}11-21-86 \\
12-03-86 \\
12-09-86 \\
12-19-86 \\
01-08-87\end{array}$ & $\begin{array}{l}78.95 \\
78.51 \\
78.28 \\
77.79 \\
76.93\end{array}$ & $\begin{array}{l}\mathbf{S} \\
\mathbf{S} \\
\mathbf{S} \\
\mathbf{S} \\
\mathbf{S}\end{array}$ \\
\hline & & & & & & $\begin{array}{l}01-20-87 \\
01-28-87 \\
02-04-87 \\
02-12-87 \\
02-19-87\end{array}$ & $\begin{array}{l}76.50 \\
76.06 \\
75.90 \\
75.63 \\
75.40\end{array}$ & $\begin{array}{l}\mathbf{S} \\
\mathbf{S} \\
\mathbf{S} \\
\mathbf{S} \\
\mathbf{S}\end{array}$ \\
\hline & & & & & & $\begin{array}{l}02-26-87 \\
03-05-87 \\
03-12-87 \\
03-20-87 \\
03-26-87\end{array}$ & $\begin{array}{l}75.11 \\
74.93 \\
74.81 \\
74.56 \\
74.44\end{array}$ & $\begin{array}{l}\text { S } \\
\text { S } \\
\text { S } \\
\text { S }\end{array}$ \\
\hline & & & & & & $\begin{array}{l}04-02-87 \\
04-09-87 \\
04-22-87 \\
04-29-87\end{array}$ & $\begin{array}{l}74.27 \\
74.18 \\
74.08 \\
74.03\end{array}$ & $\begin{array}{l}\text { S } \\
\text { S } \\
\text { S } \\
\text { S }\end{array}$ \\
\hline
\end{tabular}


TABLE 1.--Hater-level data for wells measured about weekly--Continued.

\begin{tabular}{|c|c|c|c|c|c|c|c|c|}
\hline \multicolumn{2}{|c|}{ Site designations } & \multirow{2}{*}{$\begin{array}{c} \\
\text { Water } \\
\text { use }\end{array}$} & \multirow{2}{*}{$\begin{array}{l}\text { Land- } \\
\text { surface } \\
\text { altitude } \\
\text { (feet } \\
\text { above } \\
\text { sea } \\
\text { level) }\end{array}$} & \multirow[b]{2}{*}{$\begin{array}{c}\text { Depth } \\
\text { of } \\
\text { well } \\
\text { (feet) }\end{array}$} & \multirow[b]{2}{*}{$\begin{array}{l}\text { Depth to } \\
\text { shal lowest } \\
\text { opening } \\
\text { (feet) }\end{array}$} & \multicolumn{3}{|c|}{ Water level } \\
\hline $\begin{array}{l}\text { Local USGS } \\
\text { identification; } \\
\text { well name or number; } \\
\text { and owner }\end{array}$ & $\begin{array}{l}\text { Standard } \\
\text { USGS } \\
\text { identification }\end{array}$ & & & & & Date & $\begin{array}{l}\text { Depth } \\
\text { (feet below } \\
\text { land surface) }\end{array}$ & $\begin{array}{l}\text { Measure- } \\
\text { ment } \\
\text { method }\end{array}$ \\
\hline \multirow[t]{12}{*}{$\begin{array}{l}212 \text { S20 E61 22BCDD1 } \\
\text { VALLEY VIEW / CITY } \\
\text { N.L.V. }\end{array}$} & 361141115085001 & $\mathbf{P}$ & 2,019 & 1,000 & 500 & $\begin{array}{l}02-26-86 \\
03-27-86 \\
04-09-86 \\
04-22-86 \\
05-01-86\end{array}$ & $\begin{array}{l}64.82 \\
64.85 \\
61.02 \\
60.32 \\
60.12\end{array}$ & $\begin{array}{l}\mathbf{S} \\
\mathbf{S} \\
\mathbf{S} \\
\mathbf{S} \\
\mathbf{S}\end{array}$ \\
\hline & & & & & & $\begin{array}{l}05-09-86 \\
05-19-86 \\
05-23-86 \\
05-29-86 \\
06-05-86\end{array}$ & $\begin{array}{l}59.98 \\
59.78 \\
59.80 \\
60.32 \\
61.98\end{array}$ & $\begin{array}{l}\mathbf{S} \\
\mathbf{S} \\
\mathbf{S} \\
\mathbf{S} \\
\mathbf{S}\end{array}$ \\
\hline & & & & & & $\begin{array}{l}06-12-86 \\
06-20-86 \\
06-25-86 \\
06-30-86 \\
07-03-86\end{array}$ & $\begin{array}{l}63.66 \\
65.38 \\
66.40 \\
67.02 \\
66.83\end{array}$ & $\begin{array}{l}\mathbf{S} \\
\mathbf{S} \\
\mathbf{S} \\
\mathbf{S} \\
\mathbf{S}\end{array}$ \\
\hline & & & & & & $\begin{array}{l}07-10-86 \\
07-18-86 \\
07-25-86 \\
08-01-86 \\
08-08-86\end{array}$ & $\begin{array}{l}68.40 \\
69.49 \\
70.03 \\
70.68 \\
71.18\end{array}$ & $\begin{array}{l}\mathbf{S} \\
\mathbf{S} \\
\mathbf{S} \\
\mathbf{S} \\
\mathbf{S}\end{array}$ \\
\hline & & & & & & $\begin{array}{l}08-15-86 \\
08-22-86 \\
08-29-86 \\
09-05-86 \\
09-12-86\end{array}$ & $\begin{array}{l}71.78 \\
72.29 \\
72.69 \\
72.95 \\
73.72\end{array}$ & $\begin{array}{l}\mathbf{S} \\
\mathbf{S} \\
\mathbf{S} \\
\mathbf{S} \\
\mathbf{S}\end{array}$ \\
\hline & & & & & & $\begin{array}{l}09-16-86 \\
09-19-86 \\
09-26-86 \\
10-03-86 \\
10-10-86\end{array}$ & $\begin{array}{l}73.76 \\
73.63 \\
73.76 \\
73.92 \\
73.78\end{array}$ & $\begin{array}{l}5 \\
5 \\
5 \\
S \\
5\end{array}$ \\
\hline & & & & & & $\begin{array}{l}10-17-86 \\
10-23-86 \\
10-31-86 \\
11-05-86 \\
11-14-86\end{array}$ & $\begin{array}{l}71.70 \\
71.58 \\
69.87 \\
69.40 \\
68.60\end{array}$ & $\begin{array}{l}\mathbf{S} \\
\mathbf{S} \\
\mathbf{S} \\
\mathbf{S} \\
\mathbf{S}\end{array}$ \\
\hline & & & & & & $\begin{array}{l}11-21-86 \\
11-28-86 \\
12-03-86 \\
12-09-86 \\
12-19-86\end{array}$ & $\begin{array}{l}67.90 \\
67.34 \\
66.92 \\
66.44 \\
65.98\end{array}$ & $\begin{array}{l}\mathbf{S} \\
\mathbf{S} \\
\mathbf{S} \\
\mathbf{S} \\
\mathbf{S}\end{array}$ \\
\hline & & & & & & $\begin{array}{l}12-23-86 \\
12-31-86 \\
01-08-87 \\
01-09-87 \\
01-20-87\end{array}$ & $\begin{array}{l}65.63 \\
64.99 \\
64.35 \\
64.27 \\
63.50\end{array}$ & $\begin{array}{l}\mathbf{S} \\
\mathbf{S} \\
\mathbf{S} \\
\mathbf{S} \\
\mathbf{S}\end{array}$ \\
\hline & & & & & & $\begin{array}{l}01-28-87 \\
02-04-87 \\
02-12-87 \\
02-19-87 \\
02-26-87\end{array}$ & $\begin{array}{l}62.94 \\
62.58 \\
62.13 \\
61.70 \\
62.22\end{array}$ & $\begin{array}{l}S \\
S \\
S \\
S \\
S\end{array}$ \\
\hline & & & & & & $\begin{array}{l}03-06-87 \\
03-12-87 \\
03-14-87 \\
03-26-87 \\
04-02-87\end{array}$ & $\begin{array}{l}60.87 \\
60.69 \\
60.48 \\
60.09 \\
59.86\end{array}$ & $\begin{array}{l}\mathbf{S} \\
\mathbf{S} \\
\mathbf{S} \\
\mathbf{S} \\
\mathbf{S}\end{array}$ \\
\hline & & & & & & $\begin{array}{l}04-09-87 \\
04-15-87 \\
04-22-87 \\
04-29-87\end{array}$ & $\begin{array}{l}59.65 \\
59.54 \\
59.53 \\
59.46\end{array}$ & $\begin{array}{l}\mathbf{S} \\
\mathbf{S} \\
\mathbf{S} \\
\mathbf{S}\end{array}$ \\
\hline
\end{tabular}


TABLE 1.--Water-level data for wells measured about weekly--Continued.

\begin{tabular}{|c|c|c|c|c|c|c|c|c|}
\hline \multicolumn{2}{|c|}{ Site designations } & \multirow{2}{*}{\multicolumn{2}{|c|}{$\begin{array}{c}\text { Land- } \\
\text { surface } \\
\text { altitude } \\
\text { (feet } \\
\text { above } \\
\text { Water sea } \\
\text { use level) }\end{array}$}} & \multirow[b]{2}{*}{$\begin{array}{l}\text { Depth } \\
\text { of } \\
\text { well } \\
\text { (feet) }\end{array}$} & \multirow[b]{2}{*}{$\begin{array}{l}\text { Depth to } \\
\text { shallowest } \\
\text { opening } \\
\text { (feet) }\end{array}$} & \multicolumn{3}{|c|}{ Water level } \\
\hline $\begin{array}{l}\text { Local USGS } \\
\text { identification; } \\
\text { well name or number; } \\
\text { and owner }\end{array}$ & $\begin{array}{c}\text { Standard } \\
\text { USfiS } \\
\text { identification }\end{array}$ & & & & & Date & $\begin{array}{l}\text { Depth } \\
\text { (feet below } \\
\text { land surface) }\end{array}$ & $\begin{array}{l}\text { Measure- } \\
\text { ment } \\
\text { method }\end{array}$ \\
\hline \multirow[t]{12}{*}{$\begin{array}{l}212 \text { S20 E61 24CCAB1 } \\
\text { COLLEGE PARK \#2 / } \\
\text { CITY N.L.V. }\end{array}$} & 361110115064601 & $\mathbf{P}$ & 1,840 & 500 & 186 & $\begin{array}{l}02-26-86 \\
03-27-86 \\
04-09-86 \\
04-22-86 \\
05-01-86\end{array}$ & $\begin{array}{l}8.02 \\
7.79 \\
7.34 \\
6.90 \\
6.72\end{array}$ & $\begin{array}{l}\text { S } \\
\text { S } \\
\text { S } \\
\text { S } \\
\text { S }\end{array}$ \\
\hline & & & & & & $\begin{array}{l}05-09-86 \\
05-19-86 \\
05-23-86 \\
05-29-86 \\
06-05-86\end{array}$ & $\begin{array}{l}6.88 \\
6.32 \\
6.22 \\
6.26 \\
6.87\end{array}$ & $\begin{array}{l}\mathbf{S} \\
\mathbf{S} \\
\mathbf{S} \\
\mathbf{S} \\
\mathbf{S}\end{array}$ \\
\hline & & & & & & $\begin{array}{l}06-12-86 \\
06-20-86 \\
06-25-86 \\
06-26-86 \\
07-03-86\end{array}$ & $\begin{array}{l}6.95 \\
6.99 \\
7.32 \\
7.28 \\
7.49\end{array}$ & $\begin{array}{l}\mathbf{S} \\
\mathbf{S} \\
\mathbf{S} \\
\mathbf{S} \\
\mathbf{S}\end{array}$ \\
\hline & & & & & & $\begin{array}{l}07-10-86 \\
07-18-86 \\
07-25-86 \\
08-01-86 \\
08-08-86\end{array}$ & $\begin{array}{l}9.01 \\
9.69 \\
8.25 \\
8.39 \\
8.53\end{array}$ & $\begin{array}{l}\mathbf{S} \\
\mathbf{S} \\
\mathbf{S} \\
\mathbf{S} \\
\mathbf{S}\end{array}$ \\
\hline & & & & & & $\begin{array}{l}08-15-86 \\
08-22-86 \\
08-28-86 \\
09-05-86 \\
09-16-86\end{array}$ & $\begin{array}{l}8.62 \\
8.87 \\
8.91 \\
9.11 \\
9.29\end{array}$ & $\begin{array}{l}\mathbf{S} \\
\mathbf{S} \\
\mathbf{S} \\
\mathbf{S} \\
\mathbf{S}\end{array}$ \\
\hline & & & & & & $\begin{array}{l}09-19-86 \\
09-26-86 \\
10-03-86 \\
10-10-86 \\
10-17-86\end{array}$ & $\begin{array}{l}9.37 \\
9.56 \\
9.35 \\
9.18 \\
8.87\end{array}$ & $\begin{array}{l}\mathbf{S} \\
\mathbf{S} \\
\mathbf{S} \\
\mathbf{S} \\
\mathbf{S}\end{array}$ \\
\hline & & & & & & $\begin{array}{l}10-24-86 \\
10-31-86 \\
11-05-86 \\
11-14-86 \\
11-22-86\end{array}$ & $\begin{array}{l}8.73 \\
8.39 \\
8.25 \\
7.84 \\
7.70\end{array}$ & $\begin{array}{l}\mathbf{S} \\
\mathbf{S} \\
\mathbf{S} \\
\mathbf{S} \\
\mathbf{S}\end{array}$ \\
\hline & & & & & & $\begin{array}{l}11-28-86 \\
12-02-86 \\
12-09-86 \\
12-19-86 \\
12-23-86\end{array}$ & $\begin{array}{l}7.50 \\
7.35 \\
7.14 \\
6.82 \\
6.68\end{array}$ & $\begin{array}{l}S \\
S \\
S \\
S \\
S\end{array}$ \\
\hline & & & & & & $\begin{array}{l}12-31-86 \\
01-08-87 \\
01-09-87 \\
01-20-87 \\
01-28-87\end{array}$ & $\begin{array}{l}6.47 \\
6.19 \\
5.95 \\
5.92 \\
5.63\end{array}$ & $\begin{array}{l}S \\
S \\
S \\
S \\
S\end{array}$ \\
\hline & & & & & & $\begin{array}{l}02-06-87 \\
02-11-87 \\
02-19-87 \\
02-27-87 \\
03-05-87\end{array}$ & $\begin{array}{l}5.54 \\
5.35 \\
5.18 \\
5.04 \\
4.92\end{array}$ & $\begin{array}{l}\mathbf{S} \\
\mathbf{S} \\
\mathbf{S} \\
\mathbf{S} \\
\mathbf{S}\end{array}$ \\
\hline & & & & & & $\begin{array}{l}03-12-87 \\
03-16-87 \\
03-26-87 \\
04-02-87 \\
04-09-87\end{array}$ & $\begin{array}{l}4.78 \\
4.57 \\
4.51 \\
4.39 \\
4.27\end{array}$ & $\begin{array}{l}\mathbf{S} \\
\mathbf{S} \\
\mathbf{S} \\
\mathbf{S} \\
\mathbf{S}\end{array}$ \\
\hline & & & & & & $\begin{array}{l}04-16-87 \\
04-22-87 \\
04-29-87\end{array}$ & $\begin{array}{l}4.13 \\
4.08 \\
3.92\end{array}$ & $\begin{array}{l}\mathbf{S} \\
\mathbf{S} \\
\mathbf{S}\end{array}$ \\
\hline
\end{tabular}


TABLE 1.--Hater-level data for wells measured about weekly--Cont inued.

\begin{tabular}{|c|c|c|c|c|c|c|c|c|}
\hline \multicolumn{2}{|c|}{ site designations } & \multirow{2}{*}{\multicolumn{2}{|c|}{$\begin{array}{c}\text { Land- } \\
\text { surface } \\
\text { al titude } \\
\text { (feet } \\
\text { above } \\
\text { Water sea } \\
\text { use level) }\end{array}$}} & \multirow[b]{2}{*}{$\begin{array}{l}\text { Depth } \\
\text { of } \\
\text { well } \\
\text { (feet) }\end{array}$} & \multirow[b]{2}{*}{$\begin{array}{l}\text { Depth to } \\
\text { shall lowest } \\
\text { opening } \\
\text { (feet) }\end{array}$} & \multicolumn{3}{|c|}{ Hater level } \\
\hline $\begin{array}{l}\text { Local USGS } \\
\text { identification; } \\
\text { well name or number; } \\
\text { and owner }\end{array}$ & $\begin{array}{c}\text { Standard } \\
\text { USGS } \\
\text { ident ification }\end{array}$ & & & & & Date & $\begin{array}{l}\text { Depth } \\
\text { (feet below } \\
\text { land surface) }\end{array}$ & $\begin{array}{l}\text { Measure- } \\
\text { ment } \\
\text { method }\end{array}$ \\
\hline $\begin{array}{l}212 \text { S20 E61 300DA1 } \\
\# 5 \text { / LVVWD }\end{array}$ & 361027115111401 & $\cdots$ & 2,120 & 489 & 268 & $\begin{array}{l}02-19-86 \\
03-18-86 \\
03-20-86 \\
03-25-86\end{array}$ & $\begin{array}{l}156.25 \\
169.69 \\
153.18 \\
152.4\end{array}$ & $\begin{array}{l}\mathbf{S} \\
\mathbf{S} \\
\mathbf{S} \\
T\end{array}$ \\
\hline \multirow[t]{7}{*}{$\begin{array}{l}212 \text { S20 E61 31AADD2 } \\
\# 6 \text { / LVVWD }\end{array}$} & 361014115111401 & $\mathbf{P}$ & 2,140 & 500 & 280 & $\begin{array}{l}03-18-86 \\
04-09-86 \\
04-22-86 \\
05-01-86 \\
05-08-86\end{array}$ & $\begin{array}{l}174.17 \\
208.86 \\
173.40 \\
174.92 \\
173.34\end{array}$ & $\begin{array}{l}\mathbf{S} \\
\mathbf{S} \\
\mathbf{S} \\
\mathbf{S} \\
\mathbf{S}\end{array}$ \\
\hline & & & & & & $\begin{array}{l}05-19-86 \\
05-23-86 \\
05-29-86 \\
06-06-86 \\
06-10-86\end{array}$ & $\begin{array}{l}177.70 \\
188.64 \\
177.25 \\
181.37 \\
184.28\end{array}$ & $\begin{array}{l}\mathbf{S} \\
\mathbf{S} \\
\mathbf{S} \\
\mathbf{S} \\
\mathbf{S}\end{array}$ \\
\hline & & & & & & $\begin{array}{l}06-19-86 \\
06-25-86 \\
07-03-86 \\
07-10-86 \\
07-17-86\end{array}$ & $\begin{array}{l}186.39 \\
187.82 \\
188.24 \\
190.79 \\
192.19\end{array}$ & $\begin{array}{l}\mathbf{S} \\
\mathbf{S} \\
\mathbf{S} \\
\mathbf{S} \\
\mathbf{S}\end{array}$ \\
\hline & & & & & & $\begin{array}{l}07-25-86 \\
08-01-86 \\
08-08-86 \\
08-15-86 \\
08-22-86\end{array}$ & $\begin{array}{l}194.29 \\
195.60 \\
198.29 \\
204.01 \\
198.40\end{array}$ & $\begin{array}{l}\mathbf{S} \\
\mathbf{S} \\
\mathbf{S} \\
\mathbf{S} \\
\mathbf{S}\end{array}$ \\
\hline & & & & & & $\begin{array}{l}08-29-86 \\
09-05-86 \\
09-12-86 \\
09-19-86 \\
09-26-86\end{array}$ & $\begin{array}{l}199.48 \\
201.02 \\
203.93 \\
200.09 \\
201.20\end{array}$ & $\begin{array}{l}\mathbf{S} \\
\mathbf{S} \\
\mathbf{S} \\
\mathbf{S} \\
\mathbf{S}\end{array}$ \\
\hline & & & & & & $\begin{array}{l}10-03-86 \\
10-10-86 \\
10-17-86 \\
10-23-86 \\
10-31-86\end{array}$ & $\begin{array}{l}200.98 \\
198.36 \\
199.52 \\
201.62 \\
201.32\end{array}$ & $\begin{array}{l}\text { S } \\
\text { S } \\
\text { S } \\
\text { S } \\
\mathbf{S}\end{array}$ \\
\hline & & & & & & $\begin{array}{l}03-13-87 \\
04-09-87 \\
04-16-87 \\
04-24-87 \\
04-30-87\end{array}$ & $\begin{array}{l}186.16 \\
187.43 \\
187.24 \\
187.39 \\
187.95\end{array}$ & $\begin{array}{l}S \\
S \\
S \\
S \\
S\end{array}$ \\
\hline \multirow[t]{4}{*}{$\begin{array}{l}212 \text { S20 E61 31DDBB1 } \\
\# 9 \text { / LVVWD }\end{array}$} & 360944115112701 & $P$ & 2,135 & 410 & 193.4 & $\begin{array}{l}02-28-86 \\
03-28-86 \\
03-31-86 \\
04-09-86 \\
04-30-86\end{array}$ & $\begin{array}{l}175 \\
170.08 \\
174 \\
169.67 \\
176\end{array}$ & $\begin{array}{l}A \\
S \\
A \\
S \\
A\end{array}$ \\
\hline & & & & & & $\begin{array}{l}05-01-86 \\
05-09-86 \\
05-19-86 \\
05-23-86 \\
05-29-86\end{array}$ & $\begin{array}{l}169.25 \\
169.31 \\
169.97 \\
170.47 \\
170.88\end{array}$ & $\begin{array}{l}\text { S } \\
\mathbf{S} \\
\mathbf{S} \\
\mathbf{S} \\
\mathbf{S}\end{array}$ \\
\hline & . & & & & & $\begin{array}{l}06-06-86 \\
09-30-86 \\
10-31-86 \\
11-30-86 \\
12-31-86\end{array}$ & $\begin{array}{l}171.29 \\
201 \\
199 \\
195 \\
191\end{array}$ & $\begin{array}{l}S \\
A \\
A \\
A \\
A\end{array}$ \\
\hline & & & & & & $\begin{array}{l}01-31-87 \\
02-28-87 \\
03-31-87 \\
04-30-87\end{array}$ & $\begin{array}{l}176 \\
184 \\
180 \\
179\end{array}$ & $\begin{array}{l}A \\
\hat{A} \\
A \\
A\end{array}$ \\
\hline
\end{tabular}


TABLE 1.--Water-level data for wells measured about weekly--Continued.

\begin{tabular}{|c|c|c|c|c|c|c|c|c|}
\hline \multicolumn{2}{|c|}{ Site designations } & \multirow{2}{*}{\multicolumn{2}{|c|}{$\begin{array}{cc}\text { Land- } \\
\text { surface } \\
\text { altitude } \\
\text { (feet } \\
\text { above }\end{array}$}} & \multirow[b]{2}{*}{$\begin{array}{l}\text { Depth } \\
\text { of } \\
\text { well } \\
\text { (feet) }\end{array}$} & \multirow[b]{2}{*}{$\begin{array}{l}\text { Depth to } \\
\text { shal lowest } \\
\text { opening } \\
\text { (feet) }\end{array}$} & \multicolumn{3}{|c|}{ Water level } \\
\hline $\begin{array}{l}\text { Local USGS } \\
\text { identification; } \\
\text { well name or number; } \\
\text { and owner }\end{array}$ & $\begin{array}{c}\text { Standard } \\
\text { USGS } \\
\text { identification }\end{array}$ & & & & & Date & $\begin{array}{l}\text { Depth } \\
\text { (feet below } \\
\text { land surface) }\end{array}$ & $\begin{array}{l}\text { Measure- } \\
\text { ment } \\
\text { method }\end{array}$ \\
\hline \multirow[t]{11}{*}{$\begin{array}{l}212 \text { S20 E61 31DDCB1 } \\
\# 10 / \text { LVVW }\end{array}$} & 360938115112801 & $\mathbf{P}$ & 2,138 & 1,250 & 495 & $\begin{array}{l}03-13-86 \\
03-20-86 \\
03-24-86 \\
03-25-86 \\
04-08-86\end{array}$ & $\begin{array}{l}179.17 \\
179.25 \\
177.73 \\
177.65 \\
177.89\end{array}$ & $\begin{array}{l}\mathbf{S} \\
\mathbf{S} \\
\mathbf{S} \\
\mathbf{S} \\
\mathbf{S}\end{array}$ \\
\hline & & & & & & $\begin{array}{l}04-22-86 \\
05-01-86 \\
05-09-86 \\
05-19-86 \\
05-23-86\end{array}$ & $\begin{array}{l}178.05 \\
177.96 \\
179.64 \\
181.79 \\
182.52\end{array}$ & $\begin{array}{l}\mathbf{S} \\
\mathbf{S} \\
\mathbf{S} \\
\mathbf{S} \\
\mathbf{S}\end{array}$ \\
\hline & & & & & & $\begin{array}{l}05-30-86 \\
06-06-86 \\
06-12-86 \\
06-19-86 \\
06-26-86\end{array}$ & $\begin{array}{l}183.43 \\
190.48 \\
198.25 \\
201.51 \\
203.07\end{array}$ & $\begin{array}{l}\mathbf{S} \\
\mathbf{S} \\
\mathbf{S} \\
\mathbf{S} \\
\mathbf{S}\end{array}$ \\
\hline & & & & & & $\begin{array}{l}07-03-86 \\
07-10-86 \\
07-17-86 \\
07-25-86 \\
08-04-86\end{array}$ & $\begin{array}{l}203.87 \\
204.95 \\
205.89 \\
207.93 \\
209.52\end{array}$ & $\begin{array}{l}\mathbf{s} \\
\mathbf{S} \\
\mathbf{S} \\
\mathbf{S} \\
\mathbf{S}\end{array}$ \\
\hline & & & & & & $\begin{array}{l}08-07-86 \\
08-15-86 \\
08-22-86 \\
08-29-86 \\
09-05-86\end{array}$ & $\begin{array}{l}209.82 \\
211.16 \\
210.41 \\
210.98 \\
211.55\end{array}$ & $\begin{array}{l}\mathbf{S} \\
\mathbf{S} \\
\mathbf{S} \\
\mathbf{S} \\
\mathbf{S}\end{array}$ \\
\hline & & & & & & $\begin{array}{l}09-12-86 \\
09-19-86 \\
09-26-86 \\
10-03-86 \\
10-10-86\end{array}$ & $\begin{array}{l}211.85 \\
211.74 \\
211.97 \\
210.59 \\
210.30\end{array}$ & $\begin{array}{l}\mathbf{S} \\
\mathbf{S} \\
\mathbf{S} \\
\mathbf{S} \\
\mathbf{S}\end{array}$ \\
\hline & & & & & & $\begin{array}{l}10-17-86 \\
10-23-86 \\
10-31-86 \\
11-07-86 \\
11-21-86\end{array}$ & $\begin{array}{l}209.74 \\
208.86 \\
208.53 \\
206.35 \\
203.34\end{array}$ & $\begin{array}{l}\mathbf{S} \\
\mathbf{S} \\
\mathbf{S} \\
\mathbf{S} \\
\mathbf{S}\end{array}$ \\
\hline & & & & & & $\begin{array}{l}12-05-86 \\
12-09-86 \\
12-19-86 \\
12-23-86 \\
12-31-86\end{array}$ & $\begin{array}{l}196.51 \\
193.33 \\
191.44 \\
190.87 \\
189.62\end{array}$ & $\begin{array}{l}\mathbf{S} \\
\mathbf{S} \\
\mathbf{S} \\
\mathbf{S} \\
\mathbf{S}\end{array}$ \\
\hline & & & & & & $\begin{array}{l}01-20-87 \\
01-22-87 \\
01-28-87 \\
02-06-87 \\
02-13-87\end{array}$ & $\begin{array}{l}185.99 \\
185.68 \\
184.53 \\
184.01 \\
182.83\end{array}$ & $\begin{array}{l}S \\
S \\
S \\
S \\
S\end{array}$ \\
\hline & & & & & & $\begin{array}{l}02-19-87 \\
02-27-87 \\
03-06-87 \\
03-13-87 \\
03-20-87\end{array}$ & $\begin{array}{l}182.20 \\
181.47 \\
181.57 \\
181.22 \\
180.92\end{array}$ & $\begin{array}{l}\mathbf{S} \\
\mathbf{S} \\
\mathbf{S} \\
\mathbf{S} \\
\mathbf{S}\end{array}$ \\
\hline & & & & & & $\begin{array}{l}03-27-87 \\
04-02-87 \\
04-09-87 \\
04-16-87 \\
04-24-87 \\
04-30-87\end{array}$ & $\begin{array}{l}180.56 \\
179.89 \\
179.58 \\
180.33 \\
180.37 \\
179.96\end{array}$ & $\begin{array}{l}\mathbf{S} \\
\mathbf{S} \\
\mathbf{S} \\
\mathbf{S} \\
\mathbf{S} \\
\mathbf{S}\end{array}$ \\
\hline
\end{tabular}


TABLE 1.-- Nater-level data for wells measured about weekly--Cont inued.

\begin{tabular}{|c|c|c|c|c|c|c|c|c|}
\hline \multicolumn{2}{|c|}{ Site designations } & \multirow{2}{*}{\multicolumn{2}{|c|}{$\begin{array}{c}\text { Land- } \\
\text { surface } \\
\text { altitude } \\
\text { (feet } \\
\text { above } \\
\text { Water sea } \\
\text { use level) }\end{array}$}} & \multirow[b]{2}{*}{$\begin{array}{l}\text { Depth } \\
\text { of } \\
\text { well } \\
\text { (feet) }\end{array}$} & \multirow[b]{2}{*}{$\begin{array}{l}\text { Depth to } \\
\text { shal lowest } \\
\text { opening } \\
\text { (feet) }\end{array}$} & \multicolumn{3}{|c|}{ Water level } \\
\hline $\begin{array}{l}\text { Local USGS } \\
\text { identification; } \\
\text { well name or number; } \\
\text { and owner }\end{array}$ & $\begin{array}{l}\text { Standard } \\
\text { USGS } \\
\text { identification }\end{array}$ & & & & & Date & $\begin{array}{l}\text { Depth } \\
\text { (feet below } \\
\text { land surface) }\end{array}$ & $\begin{array}{l}\text { Measure- } \\
\text { ment } \\
\text { method }\end{array}$ \\
\hline \multirow[t]{10}{*}{$\begin{array}{c}212 \text { S20 E61 34BCB1 } \\
\text { / UNION PACIFIC } \\
\text { R.R. }\end{array}$} & 361008115084101 & U & 2,034 & 780 & 280 & $\begin{array}{l}02-20-86 \\
03-27-86 \\
04-09-86 \\
05-07-86 \\
05-15-86\end{array}$ & $\begin{array}{l}66.43 \\
65.70 \\
65.60 \\
66.33 \\
67.57\end{array}$ & $\begin{array}{l}5 \\
\text { S } \\
\text { S } \\
\text { S } \\
\mathbf{S}\end{array}$ \\
\hline & & & & & & $\begin{array}{l}05-23-86 \\
05-29-86 \\
06-06-86 \\
06-12-86 \\
06-20-86\end{array}$ & $\begin{array}{l}68.43 \\
67.98 \\
68.87 \\
71.10 \\
71.19\end{array}$ & $\begin{array}{l}\mathbf{S} \\
\mathbf{S} \\
\mathbf{S} \\
\mathbf{S} \\
\mathbf{S}\end{array}$ \\
\hline & & & & & & $\begin{array}{l}06-25-86 \\
07-03-86 \\
07-10-86 \\
07-17-86 \\
08-29-86\end{array}$ & $\begin{array}{l}73.19 \\
73.79 \\
75.48 \\
76.48 \\
79.88\end{array}$ & $\begin{array}{l}\mathbf{S} \\
\mathbf{S} \\
\mathbf{S} \\
\mathbf{S} \\
\mathbf{S}\end{array}$ \\
\hline & & & & & & $\begin{array}{l}09-05-86 \\
09-12-86 \\
09-19-86 \\
09-26-86 \\
10-03-86\end{array}$ & $\begin{array}{l}82.08 \\
80.81 \\
80.89 \\
82.64 \\
81.05\end{array}$ & $\begin{array}{l}\mathbf{S} \\
\mathbf{S} \\
\mathbf{S} \\
\mathbf{S} \\
\mathbf{S}\end{array}$ \\
\hline & & & & & & $\begin{array}{l}10-10-86 \\
10-17-86 \\
10-23-86 \\
10-31-86 \\
11-07-86\end{array}$ & $\begin{array}{l}82.51 \\
81.97 \\
81.50 \\
81.78 \\
81.50\end{array}$ & $\begin{array}{l}\mathbf{S} \\
\mathbf{S} \\
\mathbf{S} \\
\mathbf{S} \\
\mathbf{S}\end{array}$ \\
\hline & & & & & & $\begin{array}{l}11-14-86 \\
11-21-86 \\
11-28-86 \\
12-05-86 \\
12-09-86\end{array}$ & $\begin{array}{l}81.28 \\
80.52 \\
80.40 \\
80.47 \\
78.98\end{array}$ & $\begin{array}{l}\mathbf{S} \\
\mathbf{S} \\
\mathbf{S} \\
\mathbf{S} \\
\mathbf{S}\end{array}$ \\
\hline & & & & & & $\begin{array}{l}12-19-86 \\
12-23-86 \\
12-31-86 \\
01-20-87 \\
01-29-87\end{array}$ & $\begin{array}{l}73.24 \\
74.35 \\
74.65 \\
74.92 \\
69.68\end{array}$ & $\begin{array}{l}\mathbf{S} \\
\mathbf{S} \\
\mathbf{S} \\
\mathbf{S} \\
\mathbf{S}\end{array}$ \\
\hline & & & & & & $\begin{array}{l}02-06-87 \\
02-13-87 \\
02-19-87 \\
02-27-87 \\
03-06-87\end{array}$ & $\begin{array}{l}70.18 \\
69.76 \\
69.57 \\
69.33 \\
69.94\end{array}$ & $\begin{array}{l}\mathbf{S} \\
\mathbf{S} \\
\mathbf{S} \\
\mathbf{S} \\
\mathbf{S}\end{array}$ \\
\hline & & & & & & $\begin{array}{l}03-13-87 \\
03-26-87 \\
04-02-87 \\
04-09-87 \\
04-16-87\end{array}$ & $\begin{array}{l}69.92 \\
69.57 \\
69.72 \\
69.93 \\
68.43\end{array}$ & $\begin{array}{l}S \\
S \\
S \\
S \\
S\end{array}$ \\
\hline & & & & & & $\begin{array}{l}04-24-87 \\
04-30-87\end{array}$ & $\begin{array}{l}65.78 \\
66.68\end{array}$ & $\begin{array}{l}\mathbf{S} \\
\mathbf{S}\end{array}$ \\
\hline
\end{tabular}


TABLE 1.--Water-level data for wells measured about weekly--Cont inued.

\begin{tabular}{|c|c|c|c|c|c|c|c|c|}
\hline \multicolumn{2}{|c|}{ Site designations } & \multirow{2}{*}{\multicolumn{2}{|c|}{$\begin{array}{c}\text { Land- } \\
\text { surface } \\
\text { altitude } \\
\text { (feet } \\
\text { above } \\
\text { Water sea } \\
\text { use level) }\end{array}$}} & \multirow[b]{2}{*}{$\begin{array}{l}\text { Depth } \\
\text { of } \\
\text { well } \\
\text { (feet) }\end{array}$} & \multirow[b]{2}{*}{$\begin{array}{l}\text { Depth to } \\
\text { shallowest } \\
\text { opening } \\
\text { (feet) }\end{array}$} & \multicolumn{3}{|c|}{ Water level } \\
\hline $\begin{array}{l}\text { Local USGS } \\
\text { identification; } \\
\text { well name or number; } \\
\text { and owner }\end{array}$ & $\begin{array}{c}\text { Standard } \\
\text { USGS } \\
\text { identification }\end{array}$ & & & & & Date & $\begin{array}{l}\text { Depth } \\
\text { (feet below } \\
\text { land surface) }\end{array}$ & $\begin{array}{l}\text { Measure- } \\
\text { ment } \\
\text { method }\end{array}$ \\
\hline \multirow[t]{11}{*}{212 S20 E61 36BCB1 } & 361006115064401 & $\mathbf{U}$ & 1,838 & $\cdots$ & $\cdots$ & $\begin{array}{l}04-10-86 \\
04-22-86 \\
05-01-86 \\
05-09-86 \\
05-19-86\end{array}$ & $\begin{array}{l}-19.2 \\
-18.8 \\
-18.6 \\
-17.5 \\
-17.3\end{array}$ & $\begin{array}{l}\mathbf{G} \\
\mathbf{G} \\
\mathbf{G} \\
\mathbf{G} \\
\mathbf{G}\end{array}$ \\
\hline & & & & & & $\begin{array}{l}05-23-86 \\
05-29-86 \\
06-05-86 \\
06-12-86 \\
06-20-86\end{array}$ & $\begin{array}{l}-17.2 \\
-16.8 \\
-16.5 \\
-15.8 \\
-15.2\end{array}$ & $\begin{array}{l}\mathbf{G} \\
\mathbf{G} \\
\mathbf{G} \\
\mathbf{G} \\
\mathbf{G}\end{array}$ \\
\hline & & & & & & $\begin{array}{l}06-25-86 \\
07-03-86 \\
07-10-86 \\
07-17-86 \\
07-25-86\end{array}$ & $\begin{array}{l}-15.0 \\
-14.5 \\
-13.8 \\
-12.8 \\
-12.5\end{array}$ & $\begin{array}{l}\mathbf{G} \\
\mathbf{G} \\
\mathbf{G} \\
\mathbf{G} \\
\mathbf{G}\end{array}$ \\
\hline & & & & & & $\begin{array}{l}08-01-86 \\
08-08-86 \\
08-15-86 \\
08-22-86 \\
08-28-86\end{array}$ & $\begin{array}{l}-12.0 \\
-11.2 \\
-11.5 \\
-11.8 \\
-11.8\end{array}$ & $\begin{array}{l}\mathbf{G} \\
\mathbf{G} \\
\mathbf{G} \\
\mathbf{G} \\
\mathbf{G}\end{array}$ \\
\hline & & & & & & $\begin{array}{l}09-05-86 \\
09-12-86 \\
09-19-86 \\
09-26-86 \\
10-03-86\end{array}$ & $\begin{array}{l}-11.0 \\
-11.0 \\
-10.8 \\
-11.0 \\
-11.2\end{array}$ & $\begin{array}{l}\mathbf{G} \\
\mathbf{G} \\
\mathbf{G} \\
\mathbf{G} \\
\mathbf{G}\end{array}$ \\
\hline & · & & & & & $\begin{array}{l}10-10-86 \\
10-17-86 \\
10-24-86 \\
10-31-86 \\
11-05-86\end{array}$ & $\begin{array}{l}-12.5 \\
-13.2 \\
-13.8 \\
-13.8 \\
-14.0\end{array}$ & $\begin{array}{l}\mathbf{G} \\
\mathbf{G} \\
\mathbf{G} \\
\mathbf{G} \\
\mathbf{G}\end{array}$ \\
\hline & & & & & & $\begin{array}{l}11-14-86 \\
11-22-86 \\
11-28-86 \\
12-02-86 \\
12-09-86\end{array}$ & $\begin{array}{l}-13.8 \\
-15.5 \\
-15.0 \\
-15.5 \\
-14.8\end{array}$ & $\begin{array}{l}\mathbf{G} \\
\mathbf{G} \\
\mathbf{G} \\
\mathbf{G} \\
\mathbf{G}\end{array}$ \\
\hline & & & & & & $\begin{array}{l}12-19-86 \\
12-23-86 \\
12-31-86 \\
01-08-87 \\
01-20-87\end{array}$ & $\begin{array}{l}-16.0 \\
-15.8 \\
-16.3 \\
-17.0 \\
-17.5\end{array}$ & $\begin{array}{l}\mathbf{G} \\
\mathbf{G} \\
\mathbf{G} \\
\mathbf{G} \\
\mathbf{G}\end{array}$ \\
\hline & & & & & & $\begin{array}{l}01-28-87 \\
02-06-87 \\
02-12-87 \\
02-20-87 \\
02-27-87\end{array}$ & $\begin{array}{l}-17.5 \\
-17.5 \\
-17.8 \\
-17.8 \\
-17.5\end{array}$ & $\begin{array}{l}\mathbf{G} \\
\mathbf{G} \\
\mathbf{G} \\
\mathbf{G}\end{array}$ \\
\hline & & & & & & $\begin{array}{l}03-05-87 \\
03-12-87 \\
03-26-87 \\
04-02-87 \\
04-09-87\end{array}$ & $\begin{array}{l}-17.5 \\
-18.0 \\
-18.3 \\
-17.8 \\
-17.5\end{array}$ & $\begin{array}{l}\mathbf{G} \\
\mathbf{G} \\
\mathbf{G} \\
\mathbf{G} \\
\mathbf{G}\end{array}$ \\
\hline & & & & & & $\begin{array}{l}04-16-87 \\
04-22-87 \\
04-29-87\end{array}$ & $\begin{array}{l}-17.0 \\
-17.3 \\
-16.8\end{array}$ & $\begin{array}{l}\mathbf{G} \\
\mathbf{G} \\
\mathbf{G}\end{array}$ \\
\hline
\end{tabular}


TABLE 1.--Water-level data for wells measured about weekly--Continued.

\begin{tabular}{|c|c|c|c|c|c|c|c|c|}
\hline \multicolumn{2}{|c|}{ site designations } & \multirow{2}{*}{\multicolumn{2}{|c|}{$\begin{array}{c}\text { Land- } \\
\text { surface } \\
\text { altitude } \\
\text { (feet } \\
\text { above } \\
\text { Water sea } \\
\text { use level) }\end{array}$}} & \multirow[b]{2}{*}{$\begin{array}{c}\text { Depth } \\
\text { of } \\
\text { well } \\
\text { (feet) }\end{array}$} & \multirow[b]{2}{*}{$\begin{array}{l}\text { Depth to } \\
\text { shal lowest } \\
\text { opening } \\
\text { (feet) }\end{array}$} & \multicolumn{3}{|c|}{ Water level } \\
\hline $\begin{array}{l}\text { Local USGS } \\
\text { identification; } \\
\text { well name or number; } \\
\text { and owner }\end{array}$ & $\begin{array}{c}\text { Standard } \\
\text { USGS } \\
\text { identification }\end{array}$ & & & & & Date & $\begin{array}{l}\text { Depth } \\
\text { (feet below } \\
\text { land surface) }\end{array}$ & $\begin{array}{l}\text { Measure- } \\
\text { ment } \\
\text { method }\end{array}$ \\
\hline \multirow[t]{11}{*}{$\begin{array}{l}212 \text { S20 E62 O3BBCC1 } \\
\text { NELLIS \#10 / U.S.A.F. }\end{array}$} & 361449115022901 & $u$ & 1,889 & 1,397 & 100 & $\begin{array}{l}03-20-86 \\
03-27-86 \\
03-28-86 \\
04-08-86 \\
04-18-86\end{array}$ & $\begin{array}{l}89.26 \\
88.93 \\
88.83 \\
88.49 \\
88.10\end{array}$ & $\begin{array}{l}\mathbf{S} \\
\mathbf{S} \\
\mathbf{S} \\
\mathbf{S} \\
\mathbf{S}\end{array}$ \\
\hline & & & & & & $\begin{array}{l}05-02-86 \\
05-09-86 \\
05-15-86 \\
05-22-86 \\
05-29-86\end{array}$ & $\begin{array}{l}88.62 \\
88.57 \\
88.39 \\
89.37 \\
89.07\end{array}$ & $\begin{array}{l}\mathbf{S} \\
\mathbf{S} \\
\mathbf{S} \\
\mathbf{S} \\
\mathbf{S}\end{array}$ \\
\hline & & & & & & $\begin{array}{l}06-05-86 \\
06-12-86 \\
06-20-86 \\
07-03-86 \\
07-10-86\end{array}$ & $\begin{array}{l}91.02 \\
93.50 \\
95.77 \\
96.81 \\
98.72\end{array}$ & $\begin{array}{l}\mathbf{S} \\
\mathbf{S} \\
\mathbf{S} \\
\mathbf{S} \\
\mathbf{S}\end{array}$ \\
\hline & & & & & & $\begin{array}{l}07-18-86 \\
07-25-86 \\
08-01-86 \\
08-08-86 \\
08-14-86\end{array}$ & $\begin{array}{l}100.82 \\
100.43 \\
100.92 \\
100.99 \\
99.61\end{array}$ & $\begin{array}{l}\mathbf{S} \\
\mathbf{S} \\
\mathbf{S} \\
\mathbf{S} \\
\mathbf{S}\end{array}$ \\
\hline & & & & & & $\begin{array}{l}08-22-86 \\
08-29-86 \\
09-05-86 \\
09-19-86 \\
09-29-86\end{array}$ & $\begin{array}{l}98.38 \\
98.42 \\
98.48 \\
97.10 \\
96.47\end{array}$ & $\begin{array}{l}\mathbf{S} \\
\mathbf{S} \\
\mathbf{S} \\
\mathbf{S} \\
\mathbf{S}\end{array}$ \\
\hline & & & & & & $\begin{array}{l}10-03-86 \\
10-10-86 \\
10-17-86 \\
10-23-86 \\
10-31-86\end{array}$ & $\begin{array}{l}95.85 \\
95.45 \\
94.80 \\
94.36 \\
93.98\end{array}$ & $\begin{array}{l}S \\
S \\
S \\
S \\
S\end{array}$ \\
\hline & & & & & & $\begin{array}{l}11-07-86 \\
11-17-86 \\
11-21-86 \\
12-03-86 \\
12-09-86\end{array}$ & $\begin{array}{l}93.24 \\
92.46 \\
92.38 \\
94.56 \\
93.18\end{array}$ & $\begin{array}{l}\mathbf{S} \\
\mathbf{S} \\
\mathbf{S} \\
\mathbf{S} \\
\mathbf{S}\end{array}$ \\
\hline & & & & & & $\begin{array}{l}12-19-86 \\
12-23-86 \\
01-02-87 \\
01-08-87 \\
01-20-87\end{array}$ & $\begin{array}{l}90.75 \\
90.56 \\
90.13 \\
90.20 \\
89.59\end{array}$ & $\begin{array}{l}S \\
S \\
S \\
S \\
S\end{array}$ \\
\hline & & & & & & $\begin{array}{l}01-22-87 \\
01-28-87 \\
02-04-87 \\
02-12-87 \\
02-19-87\end{array}$ & $\begin{array}{l}89.26 \\
89.00 \\
88.96 \\
88.71 \\
88.53\end{array}$ & $\begin{array}{l}S \\
S \\
S \\
S \\
S\end{array}$ \\
\hline & & & & & & $\begin{array}{l}02-26-87 \\
03-05-87 \\
03-12-87 \\
03-20-87 \\
04-03-87\end{array}$ & $\begin{array}{l}88.20 \\
88.14 \\
88.09 \\
87.76 \\
87.44\end{array}$ & $\begin{array}{l}S \\
S \\
S \\
S \\
S\end{array}$ \\
\hline & & & & & & $\begin{array}{l}04-08-87 \\
04-16-87 \\
04-22-87 \\
04-29-87\end{array}$ & $\begin{array}{l}87.64 \\
87.56 \\
87.30 \\
87.34\end{array}$ & $\begin{array}{l}S \\
S \\
S \\
S\end{array}$ \\
\hline
\end{tabular}


TABLE 1.--Water-level data for wells measured about weekly--Cont inued.

\begin{tabular}{|c|c|c|c|c|c|c|c|c|}
\hline \multicolumn{2}{|c|}{ site designations } & \multirow{2}{*}{\multicolumn{2}{|c|}{$\begin{array}{c}\text { Land- } \\
\text { surface } \\
\text { al titude } \\
\text { (feet } \\
\text { above } \\
\text { Water sea } \\
\text { use level) }\end{array}$}} & \multirow[b]{2}{*}{$\begin{array}{l}\text { Depth } \\
\text { of } \\
\text { well } \\
\text { (feet) }\end{array}$} & \multirow[b]{2}{*}{$\begin{array}{l}\text { Depth to } \\
\text { shall lowest } \\
\text { opening } \\
\text { (feet) }\end{array}$} & \multicolumn{3}{|c|}{ Water level } \\
\hline $\begin{array}{l}\text { Local USGS } \\
\text { identification; } \\
\text { well name or number; } \\
\text { and owner }\end{array}$ & $\begin{array}{c}\text { Standard } \\
\text { USGS } \\
\text { identification }\end{array}$ & & & & & Date & $\begin{array}{l}\text { Depth } \\
\text { (feet below } \\
\text { land surface) }\end{array}$ & $\begin{array}{l}\text { Measure- } \\
\text { ment } \\
\text { method }\end{array}$ \\
\hline
\end{tabular}

Nevada Division of Water Resources Measurements

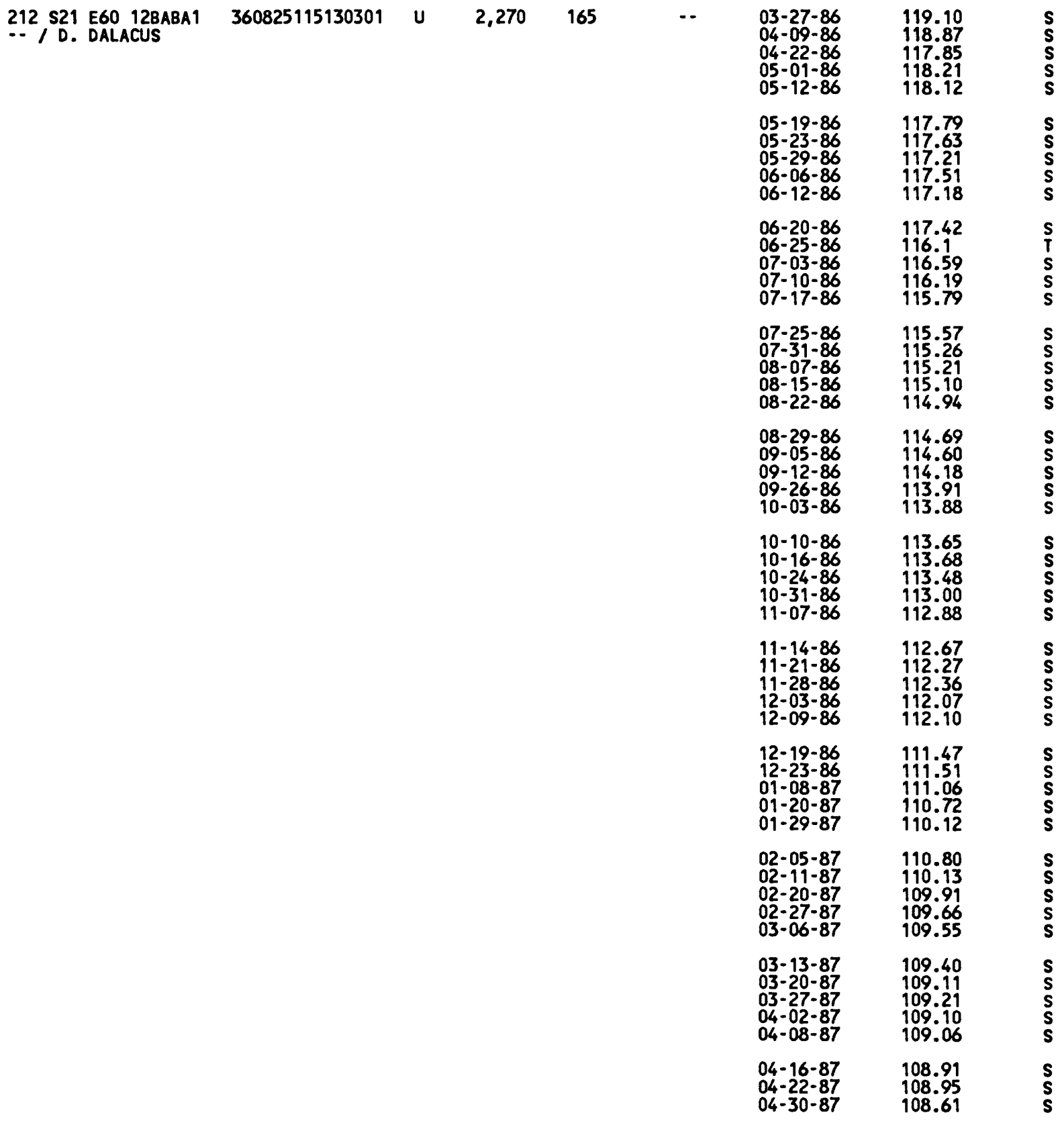


TABLE 1.--Water-level data for wells measured about weekly--Continued.

\begin{tabular}{|c|c|c|c|c|c|c|c|c|}
\hline \multicolumn{2}{|c|}{ Site designations } & \multirow{2}{*}{\multicolumn{2}{|c|}{$\begin{array}{c}\text { Land- } \\
\text { surface } \\
\text { altitude } \\
\text { (feet } \\
\text { above } \\
\text { Water sea } \\
\text { use level) }\end{array}$}} & \multirow[b]{2}{*}{$\begin{array}{l}\text { Depth } \\
\text { of } \\
\text { well } \\
\text { (feet) }\end{array}$} & \multirow[b]{2}{*}{$\begin{array}{l}\text { Depth to } \\
\text { shallowest } \\
\text { opening } \\
\text { (feet) }\end{array}$} & \multicolumn{3}{|c|}{ Water level } \\
\hline $\begin{array}{l}\text { Local USGS } \\
\text { identification; } \\
\text { well name or number; } \\
\text { and owner }\end{array}$ & $\begin{array}{l}\text { Standard } \\
\text { USGS } \\
\text { identification }\end{array}$ & & & & & Date & $\begin{array}{l}\text { Depth } \\
\text { (feet below } \\
\text { land surface) }\end{array}$ & $\begin{array}{l}\text { Measure- } \\
\text { ment } \\
\text { method }\end{array}$ \\
\hline \multirow[t]{11}{*}{$\begin{array}{l}212 \text { S21 E60 16BDDB1 } \\
\text { / CLEAR GRAVEL } \\
\text { INC. }\end{array}$} & 360712115155501 & $u$ & 2,545 & 750 & 405 & $\begin{array}{l}03-27-86 \\
04-09-86 \\
04-22-86 \\
05-01-86 \\
05-12-86\end{array}$ & $\begin{array}{l}483.5 \\
482.9 \\
491.3 \\
488.8 \\
488.3\end{array}$ & $\begin{array}{l}T \\
T \\
T \\
T \\
T\end{array}$ \\
\hline & & & & & & $\begin{array}{l}05-19-86 \\
05-23-86 \\
05-30-86 \\
06-06-86 \\
06-12-86\end{array}$ & $\begin{array}{l}488.3 \\
488.3 \\
488.4 \\
488.3 \\
488.4\end{array}$ & $\begin{array}{l}T \\
T \\
T \\
T \\
T\end{array}$ \\
\hline & & & & & & $\begin{array}{l}06-20-86 \\
06-25-86 \\
06-30-86 \\
07-03-86 \\
07-10-86\end{array}$ & $\begin{array}{l}488.4 \\
488.2 \\
489.04 \\
488.3 \\
488.3\end{array}$ & $\begin{array}{l}T \\
T \\
T \\
T \\
T\end{array}$ \\
\hline & & & & & & $\begin{array}{l}07-17-86 \\
07-25-86 \\
07-31-86 \\
08-07-86 \\
08-15-86\end{array}$ & $\begin{array}{l}489.0 \\
489.3 \\
489.0 \\
489.1 \\
489.2\end{array}$ & $\begin{array}{l}T \\
T \\
T \\
T \\
T\end{array}$ \\
\hline & & & & & & $\begin{array}{l}08-22-86 \\
08-29-86 \\
09-05-86 \\
09-10-86 \\
09-12-86\end{array}$ & $\begin{array}{l}489.3 \\
489.4 \\
489.6 \\
489.5 \\
489.30\end{array}$ & $\begin{array}{l}T \\
T \\
T \\
T \\
\text { s }\end{array}$ \\
\hline & & & & & & $\begin{array}{l}09-18-86 \\
09-26-86 \\
10-03-86 \\
10-10-86 \\
10-16-86\end{array}$ & $\begin{array}{l}489.6 \\
489.6 \\
489.6 \\
489.6 \\
489.7\end{array}$ & $\begin{array}{l}\mathbf{T} \\
\mathbf{T} \\
\mathbf{T} \\
\mathbf{T} \\
\mathbf{T}\end{array}$ \\
\hline & & & & & & $\begin{array}{l}10-24-86 \\
10-31-86 \\
11-07-86 \\
11-14-86 \\
11-21-86\end{array}$ & $\begin{array}{l}489.9 \\
489.6 \\
489.8 \\
489.8 \\
489.8\end{array}$ & $\begin{array}{l}T \\
T \\
T \\
T \\
T\end{array}$ \\
\hline & & & & & & $\begin{array}{l}11-25-86 \\
12-05-86 \\
12-19-86 \\
12-23-86 \\
12-31-86\end{array}$ & $\begin{array}{l}489.8 \\
489.9 \\
490.0 \\
490.1 \\
490.0\end{array}$ & $\begin{array}{l}T \\
T \\
T \\
T \\
T\end{array}$ \\
\hline & & & & & & $\begin{array}{l}01-06-87 \\
01-08-87 \\
01-20-87 \\
01-29-87 \\
02-05-87\end{array}$ & $\begin{array}{l}490.4 \\
490.5 \\
490.5 \\
490.2 \\
490.8\end{array}$ & $\begin{array}{l}\text { s } \\
T \\
T \\
T \\
T\end{array}$ \\
\hline & & & & & & $\begin{array}{l}02-11-87 \\
02-20-87 \\
02-27-87 \\
03-06-87 \\
03-13-87\end{array}$ & $\begin{array}{l}490.3 \\
490.6 \\
490.9 \\
490.3 \\
490.3\end{array}$ & $\begin{array}{l}T \\
T \\
T \\
T \\
T\end{array}$ \\
\hline & & & & & & $\begin{array}{l}03-17-87 \\
04-03-87 \\
04-08-87 \\
04-16-87 \\
04-22-87 \\
04-30-87\end{array}$ & $\begin{array}{l}490.7 \\
490.4 \\
491.0 \\
490.9 \\
490.7 \\
490.6\end{array}$ & $\begin{array}{l}\mathbf{S} \\
\mathbf{T} \\
\mathrm{T} \\
\mathrm{T} \\
\mathrm{T} \\
\mathrm{T}\end{array}$ \\
\hline
\end{tabular}


TABLE 1.--Hater-level data for wells measured about weekly--Continued.

\begin{tabular}{|c|c|c|c|c|c|c|c|c|}
\hline \multicolumn{2}{|c|}{ site designations } & \multirow{2}{*}{\multicolumn{2}{|c|}{$\begin{array}{cc}\text { Land- } \\
\text { surface } \\
\text { altitude } \\
\text { (feet } \\
\text { above } \\
\text { Hater sea } \\
\text { use level) }\end{array}$}} & \multirow[b]{2}{*}{$\begin{array}{l}\text { Depth } \\
\text { of } \\
\text { well } \\
\text { (feet) }\end{array}$} & \multirow[b]{2}{*}{$\begin{array}{l}\text { Depth to } \\
\text { shall owest } \\
\text { opening } \\
\text { (feet) }\end{array}$} & \multicolumn{3}{|c|}{ Hater level } \\
\hline $\begin{array}{l}\text { Local USGS } \\
\text { ident ification; } \\
\text { well name or number; } \\
\text { and owner }\end{array}$ & $\begin{array}{c}\text { Standard } \\
\text { USGS } \\
\text { identification }\end{array}$ & & & & & Date & $\begin{array}{l}\text { Depth } \\
\text { (feet below } \\
\text { land surface) }\end{array}$ & $\begin{array}{l}\text { Measure- } \\
\text { ment } \\
\text { method }\end{array}$ \\
\hline \multirow[t]{11}{*}{$\begin{array}{l}212 \text { S21 E61 03ABB2 } \\
-- \text { / DR. PARK }\end{array}$} & 360931115083802 & $\mathbf{u}$ & 2,014 & 807 & -- & $\begin{array}{l}02-20-86 \\
02-26-86 \\
03-27-86 \\
04-09-86 \\
04-22-86\end{array}$ & $\begin{array}{l}56.12 \\
56.45 \\
57.39 \\
58.03 \\
58.83\end{array}$ & $\begin{array}{l}\mathbf{S} \\
\mathbf{S} \\
\mathbf{S} \\
\mathbf{S} \\
\mathbf{S}\end{array}$ \\
\hline & & & & & & $\begin{array}{l}05-01-86 \\
05-09-86 \\
05-19-86 \\
05-23-86 \\
05-29-86\end{array}$ & $\begin{array}{l}59.66 \\
59.71 \\
60.53 \\
61.49 \\
62.32\end{array}$ & $\begin{array}{l}\mathbf{S} \\
\mathbf{S} \\
\mathbf{S} \\
\mathbf{S} \\
\mathbf{S}\end{array}$ \\
\hline & & & & & & $\begin{array}{l}06-06-86 \\
06-12-86 \\
06-20-86 \\
06-25-86 \\
06-26-86\end{array}$ & $\begin{array}{l}63.16 \\
64.05 \\
65.27 \\
66.20 \\
66.13\end{array}$ & $\begin{array}{l}\mathbf{S} \\
\mathbf{S} \\
\mathbf{S} \\
\mathbf{S} \\
\mathbf{S}\end{array}$ \\
\hline & & & & & & $\begin{array}{l}07-03-86 \\
07-10-86 \\
07-17-86 \\
07-25-86 \\
08-01-86\end{array}$ & $\begin{array}{l}67.09 \\
68.05 \\
68.89 \\
69.14 \\
69.46\end{array}$ & $\begin{array}{l}\mathbf{S} \\
\mathbf{S} \\
\mathbf{S} \\
\mathbf{S} \\
\mathbf{S}\end{array}$ \\
\hline & & & & & & $\begin{array}{l}08-08-86 \\
08-15-86 \\
08-22-86 \\
08-29-86 \\
09-05-86\end{array}$ & $\begin{array}{l}70.66 \\
71.35 \\
71.93 \\
72.02 \\
72.98\end{array}$ & $\begin{array}{l}\mathbf{S} \\
\mathbf{S} \\
\mathbf{S} \\
\mathbf{S} \\
\mathbf{S}\end{array}$ \\
\hline & & & & & & $\begin{array}{l}09-10-86 \\
09-12-86 \\
09-19-86 \\
09-26-86 \\
10-03-86\end{array}$ & $\begin{array}{l}72.66 \\
72.86 \\
72.84 \\
72.81 \\
72.75\end{array}$ & $\begin{array}{l}\mathbf{S} \\
\mathbf{S} \\
\mathbf{S} \\
\mathbf{S} \\
\mathbf{S}\end{array}$ \\
\hline & & & & & & $\begin{array}{l}10-10-86 \\
10-17-86 \\
10-23-86 \\
10-31-86 \\
11-05-86\end{array}$ & $\begin{array}{l}71.94 \\
71.87 \\
71.54 \\
70.90 \\
70.44\end{array}$ & $\begin{array}{l}\mathbf{S} \\
\mathbf{S} \\
\mathbf{S} \\
\mathbf{S} \\
\mathbf{S}\end{array}$ \\
\hline & & & & & & $\begin{array}{l}11-14-86 \\
11-22-86 \\
11-28-86 \\
12-02-86 \\
12-09-86\end{array}$ & $\begin{array}{l}69.69 \\
68.70 \\
67.79 \\
67.23 \\
65.98\end{array}$ & $\begin{array}{l}\mathbf{S} \\
\mathbf{S} \\
\mathbf{S} \\
\mathbf{S} \\
\mathbf{S}\end{array}$ \\
\hline & & & & & & $\begin{array}{l}12-19-86 \\
12-23-86 \\
12-30-86 \\
12-31-86 \\
01-08-87\end{array}$ & $\begin{array}{l}64.30 \\
63.66 \\
61.81 \\
62.45 \\
61.42\end{array}$ & $\begin{array}{l}\mathbf{S} \\
\mathbf{S} \\
\mathbf{S} \\
\mathbf{S} \\
\mathbf{S}\end{array}$ \\
\hline & & & & & & $\begin{array}{l}01-20-87 \\
01-29-87 \\
02-06-87 \\
02-13-87 \\
02-20-87\end{array}$ & $\begin{array}{l}59.80 \\
58.85 \\
58.37 \\
57.89 \\
57.59\end{array}$ & $\begin{array}{l}\mathbf{S} \\
\mathbf{S} \\
\mathbf{S} \\
\mathbf{S} \\
\mathbf{S}\end{array}$ \\
\hline & & & & & & $\begin{array}{l}02-27-87 \\
03-05-87 \\
03-13-87 \\
03-20-87 \\
03-26-87\end{array}$ & $\begin{array}{l}57.28 \\
56.86 \\
56.47 \\
56.38 \\
54.44\end{array}$ & $\begin{array}{l}\mathbf{S} \\
\mathbf{S} \\
\mathbf{S} \\
\mathbf{S} \\
\mathbf{S}\end{array}$ \\
\hline
\end{tabular}


TABLE 1.--Water-level data for wells measured about weekly--Continued.

\begin{tabular}{|c|c|c|c|c|c|c|c|c|}
\hline \multicolumn{2}{|c|}{ Site designations } & \multirow{2}{*}{\multicolumn{2}{|c|}{$\begin{array}{c}\text { Land- } \\
\text { surface } \\
\text { altitude } \\
\text { (feet } \\
\text { above } \\
\text { Water sea } \\
\text { use level) }\end{array}$}} & \multirow[b]{2}{*}{$\begin{array}{c}\text { Depth } \\
\text { of } \\
\text { well } \\
\text { (feet) }\end{array}$} & \multirow[b]{2}{*}{$\begin{array}{l}\text { Depth to } \\
\text { shall lowest } \\
\text { opening } \\
\text { (feet) }\end{array}$} & \multicolumn{3}{|c|}{ Water level } \\
\hline $\begin{array}{l}\text { Local USGS } \\
\text { identification; } \\
\text { well name or number; } \\
\text { and owner }\end{array}$ & $\begin{array}{l}\text { Standard } \\
\text { USGS } \\
\text { identification }\end{array}$ & & & & & Date & $\begin{array}{l}\text { Depth } \\
\text { (feet below } \\
\text { land surface) }\end{array}$ & $\begin{array}{l}\text { Measure- } \\
\text { ment } \\
\text { method }\end{array}$ \\
\hline \multicolumn{2}{|c|}{212 S21 E61 03ABB2--Cont inued } & & & & & $\begin{array}{l}04-02-87 \\
04-09-87 \\
04-16-87 \\
04-22-87 \\
04-30-87\end{array}$ & $\begin{array}{l}56.11 \\
56.43 \\
57.12 \\
57.99 \\
59.04\end{array}$ & $\begin{array}{l}\mathbf{S} \\
\mathbf{S} \\
\mathbf{S} \\
\mathbf{S} \\
\mathbf{S}\end{array}$ \\
\hline \multirow[t]{2}{*}{$\begin{array}{l}212 \text { S21 E61 19CBA1 } \\
- \text { / KENO RADIO }\end{array}$} & 360614115114901 & -- & 2,215 & 300 & 210 & $\begin{array}{l}03-11-86 \\
04-09-86 \\
04-22-86 \\
05-01-86 \\
05-12-86\end{array}$ & $\begin{array}{l}216.75 \\
215.7 \\
214.9 \\
212.4 \\
212.7\end{array}$ & $\begin{array}{l}\mathbf{s} \\
\mathbf{T} \\
\mathbf{T} \\
\mathbf{T} \\
\mathbf{T}\end{array}$ \\
\hline & & & & & & $\begin{array}{l}05-19-86 \\
05-23-86 \\
05-30-86\end{array}$ & $\begin{array}{l}214.8 \\
215.5 \\
215.5\end{array}$ & $\begin{array}{l}T \\
T \\
T\end{array}$ \\
\hline \multirow[t]{8}{*}{$\begin{array}{l}212 \text { S21 E61 27DDBC1 } \\
\# 43 \text { / LVVDD }\end{array}$} & 360518115082401 & $\mathbf{p}$ & 2,050 & 1,200 & 500 & $\begin{array}{l}02-19-86 \\
03-27-86 \\
04-08-86 \\
04-22-86 \\
05-01-86\end{array}$ & $\begin{array}{l}20.75 \\
22.02 \\
22.20 \\
21.79 \\
23.51\end{array}$ & $\begin{array}{l}\text { S } \\
\\
\mathbf{S} \\
\mathbf{S}\end{array}$ \\
\hline & & & & & & $\begin{array}{l}05-09-86 \\
05-19-86 \\
05-23-86 \\
05-30-86 \\
06-06-86\end{array}$ & $\begin{array}{l}24.33 \\
25.65 \\
25.69 \\
26.68 \\
26.97\end{array}$ & $\begin{array}{l}\mathbf{S} \\
\mathbf{S} \\
\mathbf{S} \\
\mathbf{S} \\
\mathbf{S}\end{array}$ \\
\hline & & & & & & $\begin{array}{l}06-12-86 \\
06-20-86 \\
06-25-86 \\
07-03-86 \\
07-10-86\end{array}$ & $\begin{array}{l}27.54 \\
28.15 \\
28.41 \\
29.02 \\
29.67\end{array}$ & $\begin{array}{l}\mathbf{S} \\
\mathbf{S} \\
\mathbf{S} \\
\mathbf{S} \\
\mathbf{S}\end{array}$ \\
\hline & & & & & & $\begin{array}{l}07-18-86 \\
07-25-86 \\
07-31-86 \\
08-04-86 \\
08-14-86\end{array}$ & $\begin{array}{l}30.04 \\
29.53 \\
29.87 \\
30.18 \\
30.62\end{array}$ & $\begin{array}{l}\mathbf{S} \\
\mathbf{S} \\
\mathbf{S} \\
\mathbf{S} \\
\mathbf{S}\end{array}$ \\
\hline & & & & & & $\begin{array}{l}08-22-86 \\
08-29-86 \\
09-05-86 \\
09-10-86 \\
09-19-86\end{array}$ & $\begin{array}{l}30.86 \\
30.46 \\
30.47 \\
30.51 \\
30.64\end{array}$ & $\begin{array}{l}\text { S } \\
\text { S } \\
\text { S } \\
\text { S } \\
\mathbf{S}\end{array}$ \\
\hline & & & & & & $\begin{array}{l}09-26-86 \\
10-03-86 \\
10-10-86 \\
10-17-86 \\
10-23-86\end{array}$ & $\begin{array}{l}29.79 \\
29.13 \\
28.85 \\
28.35 \\
28.05\end{array}$ & $\begin{array}{l}\mathbf{S} \\
\mathbf{S} \\
\mathbf{S} \\
\mathbf{S} \\
\mathbf{S}\end{array}$ \\
\hline & & & & & & $\begin{array}{l}10-31-86 \\
11-07-86 \\
11-21-86 \\
12-02-86 \\
12-04-86\end{array}$ & $\begin{array}{l}27.89 \\
27.31 \\
26.30 \\
25.42 \\
25.36\end{array}$ & $\begin{array}{l}\text { S } \\
\text { S } \\
\text { S } \\
\text { S } \\
\text { S }\end{array}$ \\
\hline & & & & & & $\begin{array}{l}12-09-86 \\
12-19-86 \\
12-23-86 \\
12-31-86 \\
01-06-87\end{array}$ & $\begin{array}{l}24.85 \\
24.00 \\
23.72 \\
23.18 \\
22.85\end{array}$ & $\begin{array}{l}\mathbf{S} \\
\mathbf{S} \\
\mathbf{S} \\
\mathbf{S} \\
\mathbf{S}\end{array}$ \\
\hline
\end{tabular}


TABLE 1.--Water-level data for wells measured about weekly--Cont inued.

\begin{tabular}{|c|c|c|c|c|c|c|c|c|}
\hline \multicolumn{2}{|c|}{ Site designations } & \multirow{2}{*}{\multicolumn{2}{|c|}{$\begin{array}{c}\text { Land- } \\
\text { surface } \\
\text { altitude } \\
\text { (feet } \\
\text { above } \\
\text { Water sea } \\
\text { use level) }\end{array}$}} & \multirow[b]{2}{*}{$\begin{array}{l}\text { Depth } \\
\text { of } \\
\text { well } \\
\text { (feet) }\end{array}$} & \multirow[b]{2}{*}{$\begin{array}{l}\text { Depth to } \\
\text { shall lowest } \\
\text { opening } \\
\text { (feet) }\end{array}$} & \multicolumn{3}{|c|}{ Water level } \\
\hline $\begin{array}{l}\text { Local USGS } \\
\text { identification; } \\
\text { well name or number; } \\
\text { and owner }\end{array}$ & $\begin{array}{l}\text { Standard } \\
\text { USGS } \\
\text { ident if ication }\end{array}$ & & & & & Date & $\begin{array}{l}\text { Depth } \\
\text { (feet below } \\
\text { land surface) }\end{array}$ & $\begin{array}{l}\text { Measure- } \\
\text { ment } \\
\text { method }\end{array}$ \\
\hline \multirow{3}{*}{\multicolumn{2}{|c|}{212 S21 E61 270DBC1--Continued }} & & & & & $\begin{array}{l}01-20-87 \\
01-29-87 \\
02-05-87 \\
02-13-87 \\
02-20-87\end{array}$ & $\begin{array}{l}22.05 \\
21.61 \\
21.60 \\
21.60 \\
21.77\end{array}$ & $\begin{array}{l}\mathbf{S} \\
\mathbf{S} \\
\mathbf{S} \\
\mathbf{S} \\
\mathbf{S}\end{array}$ \\
\hline & & & & & . & $\begin{array}{l}02-25-87 \\
03-06-87 \\
03-13-87 \\
03-27-87 \\
04-02-87\end{array}$ & $\begin{array}{l}21.72 \\
21.74 \\
21.54 \\
21.78 \\
22.01\end{array}$ & $\begin{array}{l}\mathbf{S} \\
\mathbf{S} \\
\mathbf{S} \\
\mathbf{S} \\
\mathbf{S}\end{array}$ \\
\hline & & & & & & $\begin{array}{l}04-08-87 \\
04-16-87 \\
04-22-87 \\
04-30-87\end{array}$ & $\begin{array}{l}22.36 \\
22.97 \\
23.19 \\
23.72\end{array}$ & $\begin{array}{l}\mathbf{S} \\
\mathbf{S} \\
\mathbf{S} \\
\mathbf{S}\end{array}$ \\
\hline \multirow[t]{8}{*}{$\begin{array}{l}212 \text { S21 E62 10ACAA1 } \\
\therefore \text { / NEVADA POWER }\end{array}$} & 360826115020001 & $u$ & 1,705 & 715 & 50 & $\begin{array}{l}03-13-86 \\
04-10-86 \\
05-01-86 \\
05-08-86 \\
05-14-86\end{array}$ & $\begin{array}{l}14.13 \\
14.06 \\
14.27 \\
14.42 \\
14.50\end{array}$ & $\begin{array}{l}\mathbf{S} \\
\mathbf{S} \\
\mathbf{S} \\
\mathbf{S} \\
\mathbf{S}\end{array}$ \\
\hline & & & & & & $\begin{array}{l}05-23-86 \\
05-30-86 \\
06-05-86 \\
06-12-86 \\
06-20-86\end{array}$ & $\begin{array}{l}14.54 \\
14.69 \\
14.79 \\
14.91 \\
15.00\end{array}$ & $\begin{array}{l}\mathbf{S} \\
\mathbf{S} \\
\mathbf{S} \\
\mathbf{S} \\
\mathbf{S}\end{array}$ \\
\hline & & & & & & $\begin{array}{l}06-25-86 \\
07-03-86 \\
07-10-86 \\
07-17-86 \\
07-25-86\end{array}$ & $\begin{array}{l}15.08 \\
15.15 \\
15.34 \\
15.22 \\
15.18\end{array}$ & $\begin{array}{l}\mathbf{S} \\
\mathbf{S} \\
\mathbf{S} \\
\mathbf{S} \\
\mathbf{S}\end{array}$ \\
\hline & & & & & & $\begin{array}{l}07-31-86 \\
08-08-86 \\
08-14-86 \\
08-22-86 \\
08-29-86\end{array}$ & $\begin{array}{l}15.50 \\
15.59 \\
15.66 \\
15.70 \\
15.74\end{array}$ & $\begin{array}{l}\mathbf{S} \\
\mathbf{S} \\
\mathbf{S} \\
\mathbf{S} \\
\mathbf{S}\end{array}$ \\
\hline & & & & & & $\begin{array}{l}09-05-86 \\
09-12-86 \\
09-19-86 \\
10-02-86 \\
10-10-86\end{array}$ & $\begin{array}{l}15.88 \\
15.94 \\
15.93 \\
15.95 \\
15.90\end{array}$ & $\begin{array}{l}\mathbf{S} \\
\mathbf{S} \\
\mathbf{S} \\
\mathbf{S} \\
\mathbf{S}\end{array}$ \\
\hline & & & & & & $\begin{array}{l}10-16-86 \\
10-24-86 \\
10-31-86 \\
11-06-86 \\
11-14-86\end{array}$ & $\begin{array}{l}15.89 \\
15.80 \\
15.66 \\
15.57 \\
15.48\end{array}$ & $\begin{array}{l}\mathbf{S} \\
\mathbf{S} \\
\mathbf{S} \\
\mathbf{S} \\
\mathbf{S}\end{array}$ \\
\hline & & & & & & $\begin{array}{l}11-21-86 \\
12-02-86 \\
12-09-86 \\
12-19-86 \\
01-02-87\end{array}$ & $\begin{array}{l}15.39 \\
15.21 \\
15.10 \\
14.93 \\
14.78\end{array}$ & $\begin{array}{l}\mathbf{S} \\
\mathbf{S} \\
\mathbf{S} \\
\mathbf{S} \\
\mathbf{S}\end{array}$ \\
\hline & & & & & & $\begin{array}{l}01-06-87 \\
01-20-87 \\
01-29-87 \\
02-06-87 \\
02-13-87\end{array}$ & $\begin{array}{l}14.72 \\
14.61 \\
14.57 \\
14.57 \\
14.50\end{array}$ & $\begin{array}{l}\text { S } \\
\text { S } \\
\text { S } \\
\text { S }\end{array}$ \\
\hline
\end{tabular}


TABLE 1.--Water-level data for wells measured about weekly--Cont inued.

\begin{tabular}{|c|c|c|c|c|c|c|c|c|}
\hline \multicolumn{2}{|c|}{ site designations } & \multirow{2}{*}{\multicolumn{2}{|c|}{$\begin{array}{c}\text { Land- } \\
\text { surface } \\
\text { al titude } \\
\text { (feet } \\
\text { above } \\
\text { Water sea } \\
\text { use level) }\end{array}$}} & \multirow[b]{2}{*}{$\begin{array}{l}\text { Depth } \\
\text { of } \\
\text { well } \\
\text { (feet) }\end{array}$} & \multirow[b]{2}{*}{$\begin{array}{l}\text { Depth to } \\
\text { shal lowest } \\
\text { opening } \\
\text { (feet) }\end{array}$} & \multicolumn{3}{|c|}{ Water level } \\
\hline $\begin{array}{l}\text { Local USGS } \\
\text { identification: } \\
\text { well name or number; } \\
\text { and owner }\end{array}$ & $\begin{array}{l}\text { Standard } \\
\text { USGS } \\
\text { identification }\end{array}$ & & & & & Date & $\begin{array}{l}\text { Depth } \\
\text { (feet below } \\
\text { land surface) }\end{array}$ & $\begin{array}{l}\text { Measure- } \\
\text { ment } \\
\text { method }\end{array}$ \\
\hline \multirow[t]{2}{*}{212 S21 E62 10ACAA1- } & Cont inued & & & & & $\begin{array}{l}02-20-87 \\
02-26-87 \\
03-05-87 \\
03-12-87 \\
03-27-87\end{array}$ & $\begin{array}{l}14.50 \\
14.46 \\
14.39 \\
14.32 \\
14.27\end{array}$ & $\begin{array}{l}\mathbf{S} \\
\mathbf{S} \\
\mathbf{S} \\
\mathbf{S} \\
\mathbf{S}\end{array}$ \\
\hline & & & & & & $\begin{array}{l}04-03-87 \\
04-08-87 \\
04-16-87 \\
04-22-87 \\
04-29-87\end{array}$ & $\begin{array}{l}14.08 \\
14.10 \\
14.11 \\
14.10 \\
14.17\end{array}$ & $\begin{array}{l}\mathbf{S} \\
\mathbf{S} \\
\mathbf{S} \\
\mathbf{S} \\
\mathbf{S}\end{array}$ \\
\hline \multirow[t]{8}{*}{$\begin{array}{l}212 \text { S21 E62 2000D1 } \\
\cdots / \text { L. BILLMAN }\end{array}$} & 360601115034401 & $\cdots$ & 1.720 & 500 & -- & $\begin{array}{l}03-13-86 \\
04-22-86 \\
05-08-86 \\
05-14-86 \\
05-22-86\end{array}$ & $\begin{array}{l}-56.3 \\
-56.2 \\
-55.2 \\
-54.8 \\
-55.5\end{array}$ & $\begin{array}{l}\mathbf{G} \\
\mathbf{G} \\
\mathbf{G} \\
\mathbf{G} \\
\mathbf{G}\end{array}$ \\
\hline & & & & & & $\begin{array}{l}05-23-86 \\
05-30-86 \\
06-05-86 \\
06-12-86 \\
06-20-86\end{array}$ & $\begin{array}{l}-54.2 \\
-54.8 \\
-54.8 \\
-54.5 \\
-54.0\end{array}$ & $\begin{array}{l}\mathbf{G} \\
\mathbf{G} \\
\mathbf{G} \\
\mathbf{G} \\
\mathbf{G}\end{array}$ \\
\hline & & & & & & $\begin{array}{l}06-26-86 \\
07-03-86 \\
07-10-86 \\
07-18-86 \\
07-25-86\end{array}$ & $\begin{array}{l}-54.0 \\
-53.8 \\
-53.2 \\
-53.2 \\
-54.0\end{array}$ & $\begin{array}{l}\mathbf{G} \\
\mathbf{G} \\
\mathbf{G} \\
\mathbf{G} \\
\mathbf{G}\end{array}$ \\
\hline & & & & & & $\begin{array}{l}07-31-86 \\
08-07-86 \\
08-15-86 \\
08-22-86 \\
08-28-86\end{array}$ & $\begin{array}{l}-53.8 \\
-53.2 \\
-52.8 \\
-52.2 \\
-53.2\end{array}$ & $\begin{array}{l}\mathbf{G} \\
\mathbf{G} \\
\mathbf{G} \\
\mathbf{G} \\
\mathbf{G}\end{array}$ \\
\hline & & & & & & $\begin{array}{l}09-05-86 \\
09-12-86 \\
09-19-86 \\
10-17-86 \\
10-24-86\end{array}$ & $\begin{array}{l}-52.8 \\
-52.8 \\
-52.2 \\
-53.0 \\
-53.0\end{array}$ & $\begin{array}{l}\mathbf{G} \\
\mathbf{G} \\
\mathbf{G} \\
\mathbf{G} \\
\mathbf{G}\end{array}$ \\
\hline & & & & & & $\begin{array}{l}10-31-86 \\
11-06-86 \\
11-14-86 \\
11-21-86 \\
12-02-86\end{array}$ & $\begin{array}{l}-52.2 \\
-53.0 \\
-52.8 \\
-53.3 \\
-52.8\end{array}$ & $\begin{array}{l}\mathbf{G} \\
\mathbf{G} \\
\mathbf{G} \\
\mathbf{G} \\
\mathbf{G}\end{array}$ \\
\hline & & & & & & $\begin{array}{l}12-09-86 \\
12-19-86 \\
01-02-87 \\
01-06-87 \\
01-20-87\end{array}$ & $\begin{array}{l}-55.0 \\
-56.5 \\
-57.0 \\
-57.3 \\
-57.5\end{array}$ & $\begin{array}{l}G \\
G \\
G \\
G \\
G\end{array}$ \\
\hline & & & & & & $\begin{array}{l}01-29-87 \\
02-06-87 \\
02-13-87 \\
02-26-87 \\
03-05-87 \\
03-13-87\end{array}$ & $\begin{array}{l}-56.3 \\
-57.5 \\
-58.0 \\
-58.3 \\
-58.5 \\
-58.3\end{array}$ & $\begin{array}{l}\mathbf{G} \\
\mathbf{G} \\
\mathbf{G} \\
\mathbf{G} \\
\mathbf{G} \\
\mathbf{G}\end{array}$ \\
\hline
\end{tabular}


TABLE 1.--Water-level data for wells measured about weekly--Cont inued.

\begin{tabular}{|c|c|c|c|c|c|c|c|c|}
\hline \multicolumn{2}{|c|}{ Site designations } & \multirow{2}{*}{\multicolumn{2}{|c|}{$\begin{array}{c}\text { Land- } \\
\text { surface } \\
\text { altitude } \\
\text { (feet } \\
\text { above }\end{array}$}} & \multirow[b]{2}{*}{$\begin{array}{l}\text { Depth } \\
\text { of } \\
\text { well } \\
\text { (feet) }\end{array}$} & \multirow[b]{2}{*}{$\begin{array}{l}\text { Depth to } \\
\text { shallowest } \\
\text { opening } \\
\text { (feet) }\end{array}$} & \multicolumn{3}{|c|}{ Water level } \\
\hline $\begin{array}{l}\text { Local USGS } \\
\text { identification; } \\
\text { well name or number; } \\
\text { and owner }\end{array}$ & $\begin{array}{l}\text { Standard } \\
\text { USGS } \\
\text { identification }\end{array}$ & & & & & Date & $\begin{array}{l}\text { Depth } \\
\text { (feet below } \\
\text { (and surface) }\end{array}$ & $\begin{array}{l}\text { Measure- } \\
\text { ment } \\
\text { method }\end{array}$ \\
\hline \multirow[t]{11}{*}{$\begin{array}{l}212 \text { S22 E60 010DDC1 } \\
\# 18 \text { / LVVWD }\end{array}$} & 360318115123501 & $\mathbf{u}$ & 2,363 & 1,071 & 250 & $\begin{array}{l}04-09-86 \\
04-22-86 \\
05-05-86 \\
05-09-86 \\
05-19-86\end{array}$ & $\begin{array}{l}291.4 \\
291.8 \\
292.57 \\
292.61 \\
292.63\end{array}$ & $\begin{array}{l}\mathbf{T} \\
\mathbf{T} \\
\mathbf{S} \\
\mathbf{S} \\
\mathbf{S}\end{array}$ \\
\hline & & & & & & $\begin{array}{l}05-23-86 \\
05-30-86 \\
06-06-86 \\
06-12-86 \\
06-20-86\end{array}$ & $\begin{array}{l}292.67 \\
292.69 \\
292.83 \\
292.91 \\
292.94\end{array}$ & $\begin{array}{l}\mathbf{S} \\
\mathbf{S} \\
\mathbf{S} \\
\mathbf{S} \\
\mathbf{S}\end{array}$ \\
\hline & & & & & & $\begin{array}{l}06-25-86 \\
07-03-86 \\
07-10-86 \\
07-18-86 \\
07-25-86\end{array}$ & $\begin{array}{l}292.1 \\
293.02 \\
293.04 \\
293.05 \\
293.1\end{array}$ & $\begin{array}{l}\mathbf{T} \\
\mathbf{S} \\
\mathbf{S} \\
\mathbf{S} \\
\mathbf{T}\end{array}$ \\
\hline & & & & & & $\begin{array}{l}07-31-86 \\
08-08-86 \\
08-15-86 \\
08-22-86 \\
08-28-86\end{array}$ & $\begin{array}{l}293.1 \\
293.1 \\
293.2 \\
293.4 \\
293.5\end{array}$ & $\begin{array}{l}T \\
T \\
T \\
T \\
T\end{array}$ \\
\hline & & & & & & $\begin{array}{l}09-05-86 \\
09-10-86 \\
09-18-86 \\
09-26-86 \\
10-03-86\end{array}$ & $\begin{array}{l}293.5 \\
293.7 \\
293.6 \\
293.8 \\
293.9\end{array}$ & $\begin{array}{l}T \\
T \\
T \\
T \\
T\end{array}$ \\
\hline & & & & & & $\begin{array}{l}10-10-86 \\
10-16-86 \\
10-24-86 \\
10-31-86 \\
11-07-86\end{array}$ & $\begin{array}{l}293.9 \\
293.8 \\
293.9 \\
293.8 \\
294.0\end{array}$ & $\begin{array}{l}\mathbf{T} \\
\mathbf{T} \\
\mathbf{T} \\
\mathbf{T} \\
\mathbf{T}\end{array}$ \\
\hline & & & & & & $\begin{array}{l}11-14-86 \\
11-21-86 \\
11-25-86 \\
12-05-86 \\
12-10-86\end{array}$ & $\begin{array}{l}293.9 \\
293.9 \\
294.0 \\
294.0 \\
294.1\end{array}$ & $\begin{array}{l}T \\
T \\
T \\
T \\
T\end{array}$ \\
\hline & & & & & & $\begin{array}{l}12-19-86 \\
12-23-86 \\
12-31-86 \\
01-08-87 \\
01-20-87\end{array}$ & $\begin{array}{l}293.9 \\
294.0 \\
293.9 \\
294.1 \\
294.1\end{array}$ & $\begin{array}{l}\mathbf{T} \\
\mathbf{T} \\
\mathbf{T} \\
\mathbf{T} \\
\mathbf{T}\end{array}$ \\
\hline & & & & & & $\begin{array}{l}01-29-87 \\
02-05-87 \\
02-11-87 \\
02-20-87 \\
02-27-87\end{array}$ & $\begin{array}{l}294.0 \\
294.1 \\
294.0 \\
294.3 \\
294.3\end{array}$ & $\begin{array}{l}T \\
T \\
T \\
T \\
T\end{array}$ \\
\hline & & & & & & $\begin{array}{l}03-06-87 \\
03-13-87 \\
04-03-87 \\
04-08-87\end{array}$ & $\begin{array}{l}294.1 \\
294.0 \\
294.1 \\
294.4\end{array}$ & $\begin{array}{l}T \\
\mathbf{T} \\
\mathbf{T} \\
\mathbf{T}\end{array}$ \\
\hline & & & & & & $\begin{array}{l}04-16-87 \\
04-22-87 \\
04-30-87\end{array}$ & $\begin{array}{l}294.3 \\
294.4 \\
294.4\end{array}$ & $\begin{array}{l}T \\
T \\
T\end{array}$ \\
\hline
\end{tabular}


TABLE 1.--Water-level data for wells measured about weekly--Continued.

\begin{tabular}{|c|c|c|c|c|c|c|c|c|}
\hline \multicolumn{2}{|c|}{ Site designations } & \multirow{2}{*}{\multicolumn{2}{|c|}{$\begin{array}{cc}\text { Land- } \\
\text { surface } \\
\text { al ti tude } \\
\text { ( feet } \\
\text { above } \\
\text { Water sea } \\
\text { use level) }\end{array}$}} & \multirow[b]{2}{*}{$\begin{array}{l}\text { Depth } \\
\text { of } \\
\text { well } \\
\text { (feet) }\end{array}$} & \multirow[b]{2}{*}{$\begin{array}{l}\text { Depth to } \\
\text { shallowest } \\
\text { opening } \\
\text { (feet) }\end{array}$} & \multicolumn{3}{|c|}{ Water level } \\
\hline $\begin{array}{l}\text { Local USGS } \\
\text { identification; } \\
\text { well name or number; } \\
\text { and owner }\end{array}$ & $\begin{array}{l}\text { Standard } \\
\text { USGS } \\
\text { identification }\end{array}$ & & & & & Date & $\begin{array}{l}\text { Depth } \\
\text { (feet below } \\
\text { land surface) }\end{array}$ & $\begin{array}{l}\text { Measure- } \\
\text { ment } \\
\text { method }\end{array}$ \\
\hline \multirow[t]{12}{*}{$\begin{array}{l}212 \text { \$19 E60 27BDC1 } \\
\$ 554 \text { / USGS }\end{array}$} & 361611115151301 & $u$ & 2,360 & 905 & $\cdots$ & $\begin{array}{l}02-03-86 \\
02-10-86 \\
02-18-86 \\
02-25-86 \\
03-04-86\end{array}$ & $\begin{array}{l}75.04 \\
73.98 \\
73.68 \\
73.57 \\
75.68\end{array}$ & $\begin{array}{l}\mathbf{S} \\
\mathbf{S} \\
\mathbf{S} \\
\mathbf{S} \\
\mathbf{S}\end{array}$ \\
\hline & & & & & & $\begin{array}{l}03-10-86 \\
03-13-86 \\
03-17-86 \\
03-24-86 \\
03-31-86\end{array}$ & $\begin{array}{l}75.50 \\
74.53 \\
74.96 \\
75.24 \\
77.50\end{array}$ & $\begin{array}{l}\mathbf{S} \\
\mathbf{S} \\
\mathbf{S} \\
\mathbf{S} \\
\mathbf{S}\end{array}$ \\
\hline & & & & & & $\begin{array}{l}04-07-86 \\
04-14-86 \\
04-21-86 \\
04-28-86 \\
05-05-86\end{array}$ & $\begin{array}{l}77.25 \\
79.46 \\
80.46 \\
79.40 \\
80.10\end{array}$ & $\begin{array}{l}\mathbf{S} \\
\mathbf{S} \\
\mathbf{S} \\
\mathbf{S} \\
\mathbf{S}\end{array}$ \\
\hline & & & & & & $\begin{array}{l}05-12-86 \\
05-19-86 \\
05-27-86 \\
06-02-86 \\
06-09-86\end{array}$ & $\begin{array}{l}82.00 \\
81.18 \\
82.25 \\
84.69 \\
83.15\end{array}$ & $\begin{array}{l}\mathbf{S} \\
\mathbf{S} \\
\mathbf{S} \\
\mathbf{S} \\
\mathbf{S}\end{array}$ \\
\hline & & & & & & $\begin{array}{l}06-16-86 \\
06-23-86 \\
06-25-86 \\
06-30-86 \\
07-14-86\end{array}$ & $\begin{array}{l}85.30 \\
88.67 \\
78.15 \\
86.12 \\
88.92\end{array}$ & $\begin{array}{l}\mathbf{S} \\
\mathbf{S} \\
\mathbf{S} \\
\mathbf{S} \\
\mathbf{S}\end{array}$ \\
\hline & & & & & & $\begin{array}{l}07-21-86 \\
08-04-86 \\
08-11-86 \\
08-18-86 \\
08-25-86\end{array}$ & $\begin{array}{l}87.39 \\
90.30 \\
89.55 \\
90.71 \\
89.40\end{array}$ & $\begin{array}{l}\mathbf{S} \\
\mathbf{S} \\
\mathbf{S} \\
\mathbf{S} \\
\mathbf{S}\end{array}$ \\
\hline & & & & & & $\begin{array}{l}09-02-86 \\
09-08-86 \\
09-15-86 \\
09-22-86 \\
09-29-86\end{array}$ & $\begin{array}{l}90.86 \\
91.56 \\
91.52 \\
88.90 \\
87.78\end{array}$ & $\begin{array}{l}\mathbf{S} \\
\mathbf{S} \\
\mathbf{S} \\
\mathbf{S} \\
\mathbf{S}\end{array}$ \\
\hline & & & & & & $\begin{array}{l}10-06-86 \\
10-13-86 \\
10-20-86 \\
10-27-86 \\
11-03-86\end{array}$ & $\begin{array}{l}86.29 \\
86.91 \\
85.50 \\
83.10 \\
82.21\end{array}$ & $\begin{array}{l}\mathbf{S} \\
\mathbf{S} \\
\mathbf{S} \\
\mathbf{S} \\
\mathbf{S}\end{array}$ \\
\hline & & & & & & $\begin{array}{l}11-10-86 \\
11-24-86 \\
12-01-86 \\
12-08-86 \\
12-15-86\end{array}$ & $\begin{array}{l}82.81 \\
81.82 \\
81.10 \\
79.76 \\
78.48\end{array}$ & $\begin{array}{l}\mathbf{S} \\
\mathbf{S} \\
\mathbf{S} \\
\mathbf{S} \\
\mathbf{S}\end{array}$ \\
\hline & & & & & & $\begin{array}{l}12-16-86 \\
12-22-86 \\
12-29-86 \\
01-05-87 \\
01-13-87\end{array}$ & $\begin{array}{l}79.97 \\
78.69 \\
77.35 \\
77.06 \\
76.43\end{array}$ & $\begin{array}{l}\mathbf{S} \\
\mathbf{S} \\
\mathbf{S} \\
\mathbf{S} \\
\mathbf{S}\end{array}$ \\
\hline & & & & & & $\begin{array}{l}01-20-87 \\
01-26-87 \\
02-02-87 \\
02-09-87 \\
02-17-87\end{array}$ & $\begin{array}{l}76.42 \\
76.77 \\
76.48 \\
76.48 \\
76.75\end{array}$ & $\begin{array}{l}\mathbf{S} \\
\mathbf{S} \\
\mathbf{S} \\
\mathbf{S} \\
\mathbf{S}\end{array}$ \\
\hline & & & & & & $\begin{array}{l}02-23-87 \\
03-02-87 \\
03-03-87 \\
03-09-87 \\
03-16-87\end{array}$ & $\begin{array}{l}78.24 \\
78.21 \\
82.57 \\
79.35 \\
77.76\end{array}$ & $\begin{array}{l}\mathbf{S} \\
\mathbf{S} \\
\mathbf{S} \\
\mathbf{S} \\
\mathbf{S}\end{array}$ \\
\hline
\end{tabular}


TABLE 1.--Water-level data for wells measured about weekly--Continued.

\begin{tabular}{|c|c|c|c|c|c|c|c|c|}
\hline \multicolumn{2}{|c|}{ Site designations } & \multirow{2}{*}{$\begin{array}{c} \\
\text { Water } \\
\text { use }\end{array}$} & \multirow{2}{*}{$\begin{array}{l}\text { Land- } \\
\text { surface } \\
\text { altitude } \\
\text { (feet } \\
\text { above } \\
\text { sea } \\
\text { level) }\end{array}$} & \multirow[b]{2}{*}{$\begin{array}{l}\text { Depth } \\
\text { of } \\
\text { well } \\
\text { (feet) }\end{array}$} & \multirow[b]{2}{*}{$\begin{array}{l}\text { Depth to } \\
\text { shal lowest } \\
\text { opening } \\
\text { (feet) }\end{array}$} & \multicolumn{3}{|c|}{ Water level } \\
\hline $\begin{array}{l}\text { Local USGS } \\
\text { identification; } \\
\text { well name or number; } \\
\text { and owner }\end{array}$ & $\begin{array}{l}\text { Standard } \\
\text { USGS } \\
\text { identification }\end{array}$ & & & & & Date & $\begin{array}{l}\text { Depth } \\
\text { (feet below } \\
\text { land surface) }\end{array}$ & $\begin{array}{l}\text { Measure- } \\
\text { ment } \\
\text { method }\end{array}$ \\
\hline \multirow[t]{11}{*}{$\begin{array}{l}212 \text { S22 E61 04BCB1 } \\
-- \text { / FITZPATRICK }\end{array}$} & 360349115100001 & $U$ & 2,225 & 355 & -- & $\begin{array}{l}02-03-86 \\
02-10-86 \\
02-18-86 \\
02-24-86 \\
02-26-86\end{array}$ & $\begin{array}{l}160.40 \\
159.50 \\
159.68 \\
159.33 \\
162.38\end{array}$ & $\begin{array}{l}\mathbf{S} \\
\mathbf{S} \\
\mathbf{S} \\
\mathbf{S} \\
\mathbf{S}\end{array}$ \\
\hline & & & & & & $\begin{array}{l}03-04-86 \\
03-10-86 \\
03-24-86 \\
04-02-86 \\
04-07-86\end{array}$ & $\begin{array}{l}160.91 \\
159.15 \\
160.97 \\
160.85 \\
160.40\end{array}$ & $\begin{array}{l}\mathbf{S} \\
\mathbf{S} \\
\mathbf{S} \\
\mathbf{S} \\
\mathbf{S}\end{array}$ \\
\hline & & & & & & $\begin{array}{l}04-14-86 \\
04-21-86 \\
04-28-86 \\
05-05-86 \\
05-12-86\end{array}$ & $\begin{array}{l}160.31 \\
160.34 \\
161.91 \\
163.62 \\
161.45\end{array}$ & $\begin{array}{l}\mathbf{S} \\
\mathbf{S} \\
\mathbf{S} \\
\mathbf{S}\end{array}$ \\
\hline & & & & & & $\begin{array}{l}05-19-86 \\
05-27-86 \\
06-02-86 \\
06-09-86 \\
06-17-86\end{array}$ & $\begin{array}{l}161.08 \\
161.02 \\
163.29 \\
162.52 \\
162.30\end{array}$ & $\begin{array}{l}\mathbf{S} \\
\mathbf{S} \\
\mathbf{S} \\
\mathbf{S} \\
\mathbf{S}\end{array}$ \\
\hline & & & & & & $\begin{array}{l}06-23-86 \\
06-24-86 \\
06-30-86 \\
07-21-86 \\
08-04-86\end{array}$ & $\begin{array}{l}163.87 \\
169.30 \\
163.75 \\
163.44 \\
168.03\end{array}$ & $\begin{array}{l}\mathbf{S} \\
\mathbf{S} \\
\mathbf{S} \\
\mathbf{S} \\
\mathbf{S}\end{array}$ \\
\hline & & & & & & $\begin{array}{l}08-11-86 \\
08-18-86 \\
09-02-86 \\
09-08-86 \\
09-11-86\end{array}$ & $\begin{array}{l}163.50 \\
164.66 \\
163.03 \\
163.02 \\
164.27\end{array}$ & $\begin{array}{l}\mathbf{S} \\
\mathbf{S} \\
\mathbf{S} \\
\mathbf{S} \\
\mathbf{S}\end{array}$ \\
\hline & & & & & & $\begin{array}{l}09-15-86 \\
09-22-86 \\
10-06-86 \\
10-13-86 \\
10-20-86\end{array}$ & $\begin{array}{l}164.85 \\
163.05 \\
163.32 \\
163.41 \\
164.38\end{array}$ & $\begin{array}{l}\mathbf{S} \\
\mathbf{S} \\
\mathbf{S} \\
\mathbf{S} \\
\mathbf{S}\end{array}$ \\
\hline & & & & & & $\begin{array}{l}11-03-86 \\
11-10-86 \\
11-24-86 \\
12-01-86 \\
12-08-86\end{array}$ & $\begin{array}{l}163.84 \\
163.93 \\
162.50 \\
162.81 \\
162.02\end{array}$ & $\begin{array}{l}\mathbf{S} \\
\mathbf{S} \\
\mathbf{S} \\
\mathbf{S} \\
\mathbf{S}\end{array}$ \\
\hline & & & & & & $\begin{array}{l}12-15-86 \\
12-18-86 \\
12-22-86 \\
12-29-86 \\
01-05-87\end{array}$ & $\begin{array}{l}161.75 \\
161.91 \\
161.32 \\
161.48 \\
161.44\end{array}$ & $\begin{array}{l}\mathbf{S} \\
\mathbf{S} \\
\mathbf{S} \\
\mathbf{S} \\
\mathbf{S}\end{array}$ \\
\hline & & & & & & $\begin{array}{l}01-12-87 \\
01-20-87 \\
01-26-87 \\
02-02-87 \\
02-09-87\end{array}$ & $\begin{array}{l}161.43 \\
161.88 \\
161.89 \\
160.06 \\
161.78\end{array}$ & $\begin{array}{l}\mathbf{S} \\
\mathbf{S} \\
\mathbf{S} \\
\mathbf{S} \\
\mathbf{S}\end{array}$ \\
\hline & & & & & & $\begin{array}{l}02-17-87 \\
02-23-87 \\
02-27-87 \\
03-02-87 \\
03-09-87 \\
03-16-87\end{array}$ & $\begin{array}{l}161.73 \\
160.10 \\
161.48 \\
160.91 \\
161.71 \\
161.95\end{array}$ & $\begin{array}{l}\mathbf{S} \\
\mathbf{S} \\
\mathbf{S} \\
\mathbf{S} \\
\mathbf{S} \\
\mathbf{S}\end{array}$ \\
\hline
\end{tabular}


TABLE 2.--Water-level data for wells measured about quarterly

$$
[\cdots, \text { no data available }]
$$

Site designations.--USGS identifications: see text section titled "U.S. Geological Survey site Designations." Well owner abbreviations: City N.L.V., City of North Las Vegas; LVWwD, Las Vegas Valley Water District; U.S.A.F., United States Air Force; USGS, United States Geological survey.

Water use.--Abbreviations: H, domestic; I, irrigation; N, industrial; P, public supply; U, unused. Water-level depth.--Minus sign indicates water level above land surface.

Water-level measurement method.--Abbreviations: A, air line; G, pressure gage; S, steel tape;

$T$, electric tape.

\begin{tabular}{|c|c|c|c|c|c|c|c|c|}
\hline \multicolumn{2}{|c|}{ site designations } & \multicolumn{2}{|r|}{$\begin{array}{l}\text { Land- } \\
\text { surface }\end{array}$} & \multirow[b]{2}{*}{$\begin{array}{l}\text { Depth } \\
\text { of } \\
\text { well } \\
\text { (feet) }\end{array}$} & \multirow[b]{2}{*}{$\begin{array}{l}\text { Depth to } \\
\text { shall lowest } \\
\text { opening } \\
\text { (feet) }\end{array}$} & \multicolumn{3}{|c|}{ Water level } \\
\hline $\begin{array}{l}\text { Local USGS } \\
\text { identification; } \\
\text { well name or number; } \\
\text { and owner }\end{array}$ & $\begin{array}{c}\text { Standard } \\
\text { USGS } \\
\text { identification }\end{array}$ & $\begin{array}{c}\text { Water } \\
\text { use }\end{array}$ & $\begin{array}{l}\text { (feet } \\
\text { above } \\
\text { sea } \\
\text { level) }\end{array}$ & & & Date & $\begin{array}{l}\quad \text { Depth } \\
\text { (feet below } \\
\text { land surface) }\end{array}$ & $\begin{array}{l}\text { Measure- } \\
\text { ment } \\
\text { method }\end{array}$ \\
\hline
\end{tabular}

U.S. Geological Survey Measurements

\begin{tabular}{|c|c|c|c|c|c|c|c|}
\hline $\begin{array}{l}212 \text { \$19 E61 31ADCD1 } \\
\cdots \text { B. SLYERS }\end{array}$ & 361514115112901 & H & 2,200 & 300 & 180 & $\begin{array}{l}03-12-86 \\
06-26-86 \\
09-16-86 \\
12-17-86 \\
03-17-87\end{array}$ & $\begin{array}{l}134.46 \\
141.49 \\
143.85 \\
138.57 \\
137.4\end{array}$ \\
\hline $\begin{array}{l}212 \text { S19 E61 31ADDC1 } \\
\cdots \text { / B. RNECHT }\end{array}$ & 361515115112301 & $\mathbf{u}$ & 2,185 & 100 & $\cdots$ & $\begin{array}{l}03-12-86 \\
06-26-86 \\
09-16-86 \\
12-17-86 \\
03-17-87\end{array}$ & $\begin{array}{r}98.08 \\
132.33 \\
134.82 \\
134.24 \\
133.60\end{array}$ \\
\hline $\begin{array}{c}212 \text { S19 E62 350CDC1 } \\
\text { L.M. } \$ 5 \text { / U.S.A.F. }\end{array}$ & 361451115004401 & $\mathbf{P}$ & .1867 & 838 & 370 & $\begin{array}{l}04-11-86 \\
06-25-86 \\
09-15-86 \\
01-09-87 \\
03-18-87\end{array}$ & $\begin{array}{l}99.01 \\
98.23 \\
97.38 \\
97.0 \\
96.3\end{array}$ \\
\hline $\begin{array}{l}212 \text { S20 E60 13DCCD } 1 \\
\cdots \text { / M. THOMAS }\end{array}$ & 361201115123701 & H & 2,224 & 157 & $\cdots$ & $\begin{array}{l}04-08-86 \\
06-27-86 \\
09-10-86 \\
12-17-86 \\
03-17-87\end{array}$ & $\begin{array}{l}27.35 \\
26.45 \\
24.73 \\
24.97 \\
25.17\end{array}$ \\
\hline $\begin{array}{l}212 \text { S20 E61 01ACCD1 } \\
\# 19 \text { / USGS }\end{array}$ & 361425115061901 & $\mathbf{u}$ & 1,919 & 84 & 80 & $\begin{array}{l}03-14-86 \\
06-30-86 \\
09-15-86 \\
12-17-86 \\
03-17-87\end{array}$ & $\begin{array}{l}62.20 \\
62.87 \\
64.36 \\
63.23 \\
62.75\end{array}$ \\
\hline $\begin{array}{l}212 \text { S20 E61 O3DAD2 } \\
\text { NELLIS } \# B ／ \text { U.S.A.F. }\end{array}$ & 361412115080801 & $\mathbf{P}$ & 1,973 & 913 & 150 & $\begin{array}{l}04-11-86 \\
06-26-86 \\
09-15-86 \\
01-09-87 \\
03-18-87\end{array}$ & $\begin{array}{l}61.62 \\
96.15 \\
90.00 \\
72.35 \\
62.15\end{array}$ \\
\hline $\begin{array}{l}212 \text { S20 E61 11CDDC1 } \\
\# 16 / \text { USGS }\end{array}$ & 361305115073201 & $\mathbf{u}$ & 1,920 & 62 & 58 & $\begin{array}{l}03-14-86 \\
06-26-86 \\
09-16-86 \\
12-22-86 \\
03-17-87\end{array}$ & $\begin{array}{l}46.99 \\
43.80 \\
42.69 \\
43.26 \\
42.79\end{array}$ \\
\hline $\begin{array}{l}212 \text { S20 E61 } 14 \mathrm{CCCC} 1 \\
\# 15 \text { / USGS }\end{array}$ & 361212115065901 & $\mathbf{u}$ & 1,910 & 46 & 43 & $\begin{array}{l}03-14-86 \\
06-26-86 \\
09-16-86 \\
01-06-87 \\
03-17-87\end{array}$ & $\begin{array}{l}29.28 \\
29.70 \\
29.71 \\
29.59 \\
29.42\end{array}$ \\
\hline
\end{tabular}


TABLE 2.--Hater-level data for wells measured about quarterly--Continued.

\begin{tabular}{|c|c|c|c|c|c|c|c|c|}
\hline \multicolumn{2}{|c|}{ Site designations } & \multirow{2}{*}{\multicolumn{2}{|c|}{$\begin{array}{c}\text { Land- } \\
\text { surface } \\
\text { altitude } \\
\text { (feet } \\
\text { above } \\
\text { Hater sea } \\
\text { use level) }\end{array}$}} & \multirow[b]{2}{*}{$\begin{array}{l}\text { Depth } \\
\text { of } \\
\text { well } \\
\text { (feet) }\end{array}$} & \multirow[b]{2}{*}{$\begin{array}{l}\text { Depth to } \\
\text { shal lowest } \\
\text { opening } \\
\text { (feet) }\end{array}$} & \multicolumn{3}{|c|}{ Hater level } \\
\hline $\begin{array}{l}\text { Local USGS } \\
\text { identification; } \\
\text { well name or number; } \\
\text { and owner }\end{array}$ & $\begin{array}{c}\text { Standard } \\
\text { USGS } \\
\text { identification }\end{array}$ & & & & & Date & $\begin{array}{l}\text { Depth } \\
\text { (feet below } \\
\text { (and surface) }\end{array}$ & $\begin{array}{l}\text { Measure- } \\
\text { ment } \\
\text { method }\end{array}$ \\
\hline $\begin{array}{l}212 \text { S20 E61 18BCCD1 } \\
\text { AIRPORT / CITY } \\
\text { N.L.V. }\end{array}$ & 361237115121401 & $\mathbf{P}$ & 2,208 & 500 & 300 & $\begin{array}{l}03-12-86 \\
06-30-86 \\
09-10-86 \\
12-17-86 \\
03-03-87\end{array}$ & $\begin{array}{l}233.43 \\
233.70 \\
228.40 \\
220.95 \\
234.4\end{array}$ & $\begin{array}{l}\mathbf{S} \\
\mathbf{S} \\
\mathbf{S} \\
\mathbf{S} \\
\mathbf{S}\end{array}$ \\
\hline $\begin{array}{l}212 \text { S20 E61 220ACD1 } \\
\text { TONOPAH / CITY } \\
\text { N.L.V. }\end{array}$ & 361120115080401 & $\mathbf{P}$ & 1,911 & 1,105 & 249 & $\begin{array}{l}03-14-86 \\
06-30-86 \\
09-16-86 \\
01-09-87 \\
03-16-87\end{array}$ & $\begin{array}{l}19.40 \\
21.56 \\
25.2 \\
12.58 \\
10.27\end{array}$ & $\begin{array}{l}S \\
T \\
T \\
S \\
S\end{array}$ \\
\hline $\begin{array}{l}212 \text { S20 E61 27BDAA1 } \\
\# 49 \text { / USGS }\end{array}$ & 361102115083601 & $u$ & 2,010 & 15 & 11 & $\begin{array}{l}03-14-86 \\
06-26-86 \\
09-16-86 \\
01-07-87 \\
03-16-87\end{array}$ & $\begin{array}{l}12.23 \\
12.23 \\
11.54 \\
10.57 \\
11.22\end{array}$ & $\begin{array}{l}\text { S } \\
\text { S } \\
\mathbf{S} \\
\mathbf{S} \\
\mathbf{S}\end{array}$ \\
\hline $\begin{array}{l}212 \text { S20 E61 30ACC1 } \\
\# 34 \text { / USGS }\end{array}$ & 361053115120501 & $u$ & 2,000 & 31 & 27 & $\begin{array}{l}03-12-86 \\
06-30-86 \\
09-10-86 \\
01-07-87 \\
03-20-87\end{array}$ & $\begin{array}{l}10.17 \\
8.48 \\
8.04 \\
7.93 \\
9.18\end{array}$ & $\begin{array}{l}\mathbf{S} \\
\mathbf{S} \\
\mathbf{S} \\
\mathbf{S} \\
\mathbf{S}\end{array}$ \\
\hline $\begin{array}{l}212 \text { S20 E61 310CD } 1 \\
\# 37 \text { / USGS }\end{array}$ & 360937115113401 & $\mathbf{u}$ & 2,155 & 18 & 14 & $\begin{array}{l}03-13-86 \\
06-25-86 \\
09-10-86 \\
12-18-86 \\
03-02-87\end{array}$ & $\begin{array}{l}9.62 \\
9.22 \\
8.17 \\
8.66 \\
9.11\end{array}$ & $\begin{array}{l}\text { S } \\
\text { S } \\
\text { S } \\
\text { S } \\
\text { S }\end{array}$ \\
\hline $\begin{array}{l}212 \text { S20 E61 32CDC1 } \\
--/ K . \text { SEARLES }\end{array}$ & 360941115104801 & H & 2,102 & 665 & 570 & $\begin{array}{l}03-10-86 \\
06-25-86 \\
09-10-86 \\
12-18-86 \\
03-03-87\end{array}$ & $\begin{array}{l}55.75 \\
65.44 \\
67.80 \\
58.00 \\
53.6\end{array}$ & $\begin{array}{l}\text { S } \\
\text { S } \\
\text { S } \\
\text { S } \\
\text { S }\end{array}$ \\
\hline $\begin{array}{l}212 \text { S20 E61 34CAA1 } \\
\# 48 / \text { USGS }\end{array}$ & 360837115095501 & $u$ & 2,010 & 22 & 18 & $\begin{array}{l}03-19-86 \\
06-26-86 \\
09-16-86 \\
12-30-86 \\
03-16-87\end{array}$ & $\begin{array}{l}6.75 \\
7.55 \\
7.17 \\
5.41 \\
5.23\end{array}$ & $\begin{array}{l}\mathbf{S} \\
\mathbf{S} \\
\mathbf{S} \\
\mathbf{S} \\
\mathbf{S}\end{array}$ \\
\hline $\begin{array}{l}212 \text { S20 E62 09CCC1 } \\
\text { NELL IS "A" } \# 14 \text { / } \\
\text { U.S. ARMY CORPS } \\
\text { OF ENGINEERS }\end{array}$ & 361258115032101 & $\mathbf{P}$ & 1,827 & 650 & 290 & $\begin{array}{l}04-11-86 \\
09-15-86 \\
09-22-86 \\
01-09-87 \\
03-18-87\end{array}$ & $\begin{array}{r}86.85 \\
167.70 \\
123.62 \\
87.77 \\
82.2\end{array}$ & \begin{tabular}{l}
$T$ \\
1 \\
\hdashline- \\
$\dot{T}$
\end{tabular} \\
\hline 212 S20 E62 290CAB1 & 361036115040401 & $u$ & 1,766 & 98 & $\cdots$ & $\begin{array}{l}03-13-86 \\
06-30-86 \\
09-11-86 \\
01-07-87 \\
03-16-87\end{array}$ & $\begin{array}{l}40.04 \\
39.96 \\
40.08 \\
39.65 \\
39.07\end{array}$ & $\begin{array}{l}\mathbf{S} \\
\mathbf{S} \\
\mathbf{S} \\
\mathbf{S} \\
\mathbf{S}\end{array}$ \\
\hline
\end{tabular}


TABLE 2.--Water-level data for wells measured about quarterly--Continued.

\begin{tabular}{|c|c|c|c|c|c|c|c|c|}
\hline \multicolumn{2}{|c|}{ site designations } & \multirow{2}{*}{\multicolumn{2}{|c|}{$\begin{array}{c}\text { Land- } \\
\text { surface } \\
\text { altitude } \\
\text { (feet } \\
\text { above } \\
\text { Water sea } \\
\text { use level) }\end{array}$}} & \multirow[b]{2}{*}{$\begin{array}{c}\text { Depth } \\
\text { of } \\
\text { well } \\
\text { (feet) }\end{array}$} & \multirow[b]{2}{*}{$\begin{array}{l}\text { Depth to } \\
\text { shall lowest } \\
\text { opening } \\
\text { (feet) }\end{array}$} & \multicolumn{3}{|c|}{ Water level } \\
\hline $\begin{array}{l}\text { Local USGS } \\
\text { identification; } \\
\text { well name or number: } \\
\text { and owner }\end{array}$ & $\begin{array}{c}\text { Standard } \\
\text { USGS } \\
\text { ident if ication }\end{array}$ & & & & & Date & $\begin{array}{l}\text { Depth } \\
\text { (feet below } \\
\text { land surface) }\end{array}$ & $\begin{array}{l}\text { Measure- } \\
\text { ment } \\
\text { method }\end{array}$ \\
\hline $\begin{array}{l}212 \text { S21 E60 15BBDC1 } \\
\cdots-\text { I WELLS CARGO }\end{array}$ & 360739115152701 & N & 2,480 & 680 & 380 & $\begin{array}{l}09-12-86 \\
01-07-87 \\
03-17-87\end{array}$ & $\begin{array}{l}432.00 \\
434.0 \\
432.5\end{array}$ & $\begin{array}{l}\mathbf{s} \\
\mathbf{T} \\
\mathbf{T}\end{array}$ \\
\hline $\begin{array}{l}212 \text { S21 E61 01ACCC1 } \\
\# 11 \text { / USGS }\end{array}$ & 360908115062901 & $u$ & 1.840 & 23.5 & 19.5 & $\begin{array}{l}03-13-86 \\
06-25-86 \\
09-15-86 \\
01-07-87 \\
03-16-87\end{array}$ & $\begin{array}{l}7.59 \\
7.60 \\
7.75 \\
7.23 \\
7.25\end{array}$ & $\begin{array}{l}\mathbf{S} \\
\mathbf{S} \\
\mathbf{S} \\
\mathbf{S} \\
\mathbf{S}\end{array}$ \\
\hline $\begin{array}{l}212 \text { S21 E61 03AAAD1 } \\
\# 47 / \text { USGS }\end{array}$ & 360924115081101 & $\mathbf{u}$ & 1,990 & 15 & 11 & $\begin{array}{l}03-14-86 \\
06-26-86 \\
09-10-86 \\
12-30-86 \\
03-18-87\end{array}$ & $\begin{array}{l}7.41 \\
7.50 \\
7.60 \\
7.51 \\
7.38\end{array}$ & $\begin{array}{l}\mathbf{S} \\
\mathbf{S} \\
\mathbf{S} \\
\mathbf{S} \\
\mathbf{S}\end{array}$ \\
\hline $\begin{array}{l}212 \text { S21 E61 O4ABC1 } \\
\# 43 \text { / USGS }\end{array}$ & 360921115093601 & $\mathbf{U}$ & 2,047 & 17 & 13 & $\begin{array}{l}03-14-86 \\
06-25-86 \\
09-10-86 \\
12-18-86 \\
03-18-87\end{array}$ & $\begin{array}{r}9.16 \\
9.66 \\
10.02 \\
9.57 \\
9.14\end{array}$ & $\begin{array}{l}\mathbf{S} \\
\mathbf{S} \\
\mathbf{S} \\
\mathbf{S} \\
\mathbf{S}\end{array}$ \\
\hline $\begin{array}{l}212 \text { S21 E61 14ACA1 } \\
\cdots \text { / SAHARA } \\
\text { COUNTRY CLUB }\end{array}$ & 360728115072901 & $\mathfrak{l}$ & 1,930 & 750 & 500 & $\begin{array}{l}03-14-86 \\
01-07-87\end{array}$ & $\begin{array}{l}26.44 \\
31.08\end{array}$ & $\mathbf{S}$ \\
\hline $\begin{array}{l}212 \text { S21 E61 16CA3 } \\
-- \text { / SANDS }\end{array}$ & 360719115095903 & $\mathbf{P}$ & 2,090 & 840 & 260 & $\begin{array}{l}03-20-86 \\
09-19-86 \\
01-07-87 \\
03-18-87\end{array}$ & $\begin{array}{l}125.83 \\
171.50 \\
115.25 \\
129.96\end{array}$ & $\begin{array}{l}\mathbf{S} \\
\mathbf{S} \\
\mathbf{S} \\
\mathbf{S}\end{array}$ \\
\hline 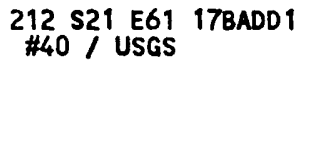 & 360735115105201 & $u$ & 2,120 & 45 & 41 & $\begin{array}{l}03-18-86 \\
06-25-86 \\
09-16-86 \\
01-07-87 \\
03-18-87\end{array}$ & $\begin{array}{l}21.80 \\
20.02 \\
17.18 \\
16.03 \\
14.09\end{array}$ & $\begin{array}{l}\mathbf{S} \\
\mathbf{S} \\
\mathbf{S} \\
\mathbf{S} \\
\mathbf{S}\end{array}$ \\
\hline $\begin{array}{l}212 \text { S21 E61 22CCC1 } \\
-- \text { / A.P. BAKER }\end{array}$ & 360600115091001 & $\mathbf{U}$ & 2,072 & 500 & - & $\begin{array}{l}03-14-86 \\
06-26-86 \\
09-12-86 \\
12-19-86 \\
03-02-87\end{array}$ & $\begin{array}{l}51.97 \\
84.70 \\
84.02 \\
50.8 \\
51.4\end{array}$ & $\begin{array}{l}\mathbf{S} \\
\mathbf{S} \\
\mathbf{S} \\
\mathbf{T} \\
\mathbf{T}\end{array}$ \\
\hline $\begin{array}{l}212 \text { S21 E61 24CAD1 } \\
\# 56 / \text { USGS }\end{array}$ & 360617115063801 & $U$ & 1,950 & 23.8 & 19.8 & $\begin{array}{l}03-14-86 \\
06-25-86 \\
09-12-86 \\
12-29-86 \\
03-02-87\end{array}$ & $\begin{array}{l}13.86 \\
13.62 \\
13.10 \\
12.87 \\
13.20\end{array}$ & $\begin{array}{l}\mathbf{S} \\
\mathbf{S} \\
\mathbf{S} \\
\mathbf{S} \\
\mathbf{S}\end{array}$ \\
\hline 212 S21 E61 28CABB1 & 360528115094201 & $\cdots$ & 2,125 & 93 & $\cdots$ & $\begin{array}{l}03-14-86 \\
06-26-86 \\
09-12-86 \\
12-19-86 \\
02-27-87\end{array}$ & $\begin{array}{l}35.53 \\
35.53 \\
35.00 \\
34.2 \\
34.2\end{array}$ & $\begin{array}{l}\mathbf{S} \\
\mathbf{S} \\
\mathbf{S} \\
\mathbf{T} \\
\mathbf{T}\end{array}$ \\
\hline
\end{tabular}


TABLE 2.--Water-level data for wells measured about quarterly--Continued.

\begin{tabular}{|c|c|c|c|c|c|c|c|c|}
\hline \multicolumn{2}{|c|}{ Site designations } & \multirow{2}{*}{\multicolumn{2}{|c|}{$\begin{array}{cc}\text { Land- } \\
\text { surface } \\
\text { al titude } \\
\text { (feet } \\
\text { above } \\
\text { Water sea } \\
\text { use level) }\end{array}$}} & \multirow[b]{2}{*}{$\begin{array}{l}\text { Depth } \\
\text { of } \\
\text { well } \\
\text { (feet) }\end{array}$} & \multirow[b]{2}{*}{$\begin{array}{l}\text { Depth to } \\
\text { shal lowest } \\
\text { opening } \\
\text { (feet) }\end{array}$} & \multicolumn{3}{|c|}{ Water level } \\
\hline $\begin{array}{l}\text { Local USGS } \\
\text { identification; } \\
\text { well name or number; } \\
\text { and owner }\end{array}$ & $\begin{array}{c}\text { Standard } \\
\text { USGS } \\
\text { ident ification }\end{array}$ & & & & & Date & $\begin{array}{l}\text { Depth } \\
\text { (feet below } \\
\text { land surface) }\end{array}$ & $\begin{array}{l}\text { Measure- } \\
\text { ment } \\
\text { method }\end{array}$ \\
\hline $\begin{array}{l}212 \text { S21 E61 29AACA1 } \\
\cdots \text { / M. WOLLMAN }\end{array}$ & 360543115101301 & $\mathbf{U}$ & 2,140 & 540 & -- & $\begin{array}{l}03-14-86 \\
06-26-86 \\
09-12-86 \\
12-19-86 \\
02-27-87\end{array}$ & $\begin{array}{c}88.38 \\
104.99 \\
109.46 \\
85.3 \\
87.5\end{array}$ & $\begin{array}{l}\mathbf{S} \\
\mathbf{S} \\
\mathbf{S} \\
\mathbf{T} \\
\mathbf{T}\end{array}$ \\
\hline $\begin{array}{l}212 \text { S21 E61 36ADC3 } \\
=68 \text { / USGS }\end{array}$ & 360449115061201 & $\mathbf{u}$ & 1,948 & 25.9 & 22.9 & $\begin{array}{l}03-07-86 \\
06-25-86 \\
09-11-86 \\
12-29-86 \\
03-02-87\end{array}$ & $\begin{array}{l}21.26 \\
23.15 \\
25.39 \\
22.14 \\
20.83\end{array}$ & $\begin{array}{l}\mathbf{S} \\
\mathbf{S} \\
\mathbf{S} \\
\mathbf{S} \\
\mathbf{S}\end{array}$ \\
\hline $\begin{array}{l}212 \text { s21 E62 08DBDA2 } \\
\cdots \text { / D. OKELLY }\end{array}$ & 360733115034402 & H & 1,731 & 200 & 50 & $\begin{array}{l}04-11-86 \\
06-26-86 \\
09-11-86 \\
01-07-87 \\
03-16-87\end{array}$ & $\begin{array}{l}14.23 \\
14.95 \\
15.89 \\
13.84 \\
12.27\end{array}$ & $\begin{array}{l}\mathbf{s} \\
\mathbf{S} \\
\mathbf{S} \\
\mathbf{S} \\
\mathbf{S}\end{array}$ \\
\hline $\begin{array}{l}212 \text { S21 E62 1TOAB1 } \\
\# 5 \text { / USGS }\end{array}$ & 360744115050801 & $\mathbf{U}$ & 1,730 & 11 & 7 & $\begin{array}{l}03-13-86 \\
06-26-86 \\
09-11-86 \\
01-07-87 \\
03-16-87\end{array}$ & $\begin{array}{l}7.05 \\
7.30 \\
8.32 \\
7.32 \\
6.27\end{array}$ & $\begin{array}{l}\mathbf{S} \\
\mathbf{S} \\
\mathbf{S} \\
\mathbf{S} \\
\mathbf{S}\end{array}$ \\
\hline $\begin{array}{c}212 \text { S21 E62 28AAC1 } \\
\# 3 \text { / USGS }\end{array}$ & 360548115024601 & $\mathbf{U}$ & 1,665 & 27 & 23 & $\begin{array}{l}03-07-86 \\
06-25-86 \\
09-15-86 \\
12-29-86 \\
03-02-87\end{array}$ & $\begin{array}{l}19.15 \\
18.65 \\
17.99 \\
18.52 \\
18.42\end{array}$ & $\begin{array}{l}\text { S } \\
\text { S } \\
\text { S } \\
\mathbf{S} \\
\mathbf{S}\end{array}$ \\
\hline $\begin{array}{c}212 \text { S22 EGO 20CACA1 } \\
\cdots \text { / MOFFAT \& } \\
\text { LILLIS }\end{array}$ & 360047115171401 & $\mathbf{u}$ & 2,810 & 710 & 610 & $\begin{array}{l}03-13-86 \\
06-24-86 \\
09-10-86 \\
12-18-86 \\
03-02-87\end{array}$ & $\begin{array}{l}477.48 \\
493.87 \\
479.90 \\
478.3 \\
478.2\end{array}$ & $\begin{array}{l}\mathbf{S} \\
\mathbf{S} \\
\mathbf{S} \\
\mathbf{T} \\
\mathbf{T}\end{array}$ \\
\hline $\begin{array}{l}212 \text { S22 E61 01CCC1 } \\
\# 66 \text { / USGS }\end{array}$ & 360328115065501 & $\mathbf{U}$ & 2,032 & 55 & 51 & $\begin{array}{l}03-07-86 \\
06-25-86 \\
03-02-87\end{array}$ & $\begin{array}{l}49.03 \\
52.33 \\
50.91\end{array}$ & $\begin{array}{l}\mathbf{S} \\
\mathbf{S} \\
\mathbf{S}\end{array}$ \\
\hline 212 S22 E61 04ACAD1 & 360400115092401 & $\cdots$ & 2,165 & 113 & $\cdots$ & $\begin{array}{l}03-13-86 \\
06-26-86 \\
09-12-86 \\
12-18-86 \\
02-27-87\end{array}$ & $\begin{array}{l}97.11 \\
95.04 \\
95.52 \\
95.94 \\
96.2\end{array}$ & $\begin{array}{l}S \\
S \\
S \\
T \\
T\end{array}$ \\
\hline $\begin{array}{l}212 \text { S22 E61 10CCD1 } \\
\cdots / \text { L.J. DEATCH }\end{array}$ & 360235115090301 & H & 2,160 & 300 & 168 & $\begin{array}{l}04-08-86 \\
06-27-86 \\
09-11-86 \\
01-09-87 \\
02-27-87\end{array}$ & $\begin{array}{l}106.90 \\
125.65 \\
126.0 \\
115.58 \\
115.85\end{array}$ & $\begin{array}{l}\mathbf{S} \\
\mathbf{S} \\
\mathbf{T} \\
\mathbf{S} \\
\mathbf{S}\end{array}$ \\
\hline $\begin{array}{l}212 \text { S22 E62 O4DCCC1 } \\
\cdots / \text { CITY OF } \\
\text { HENDERSON }\end{array}$ & 360322115030801 & $\cdots$ & 1,798 & 780 & 430 & $\begin{array}{l}03-13-86 \\
06-03-86 \\
09-19-86\end{array}$ & $\begin{array}{l}-5.5 \\
-5.5 \\
-2.3\end{array}$ & $\begin{array}{l}\mathbf{G} \\
\mathbf{G} \\
\mathbf{G}\end{array}$ \\
\hline $\begin{array}{c}212 \text { S22 E63 20ABC1 } \\
\cdots / \text { CIIY OF } \\
\text { HENDERSON }\end{array}$ & 360122114574801 & $\mathbf{u}$ & 2,030 & 750 & 460 & $\begin{array}{l}03-07-86 \\
06-25-86 \\
09-11-86 \\
12-17-86 \\
02-27-87\end{array}$ & $\begin{array}{l}315.24 \\
314.92 \\
315.20 \\
314.50 \\
314.6\end{array}$ & $\begin{array}{l}S \\
S \\
S \\
S \\
T\end{array}$ \\
\hline
\end{tabular}


TABLE 2.--Water-level data for wells measured about quarterly--Continued.

\begin{tabular}{|c|c|c|c|c|c|c|c|c|}
\hline \multicolumn{2}{|c|}{ site designations } & \multirow{2}{*}{\multicolumn{2}{|c|}{$\begin{array}{c}\text { Land- } \\
\text { surface } \\
\text { al titude } \\
\text { (feet } \\
\text { above } \\
\text { Water sea } \\
\text { use level) }\end{array}$}} & \multirow[b]{2}{*}{$\begin{array}{l}\text { Depth } \\
\text { of } \\
\text { well } \\
\text { (feet) }\end{array}$} & \multirow[b]{2}{*}{$\begin{array}{l}\text { Depth to } \\
\text { shallowest } \\
\text { opening } \\
\text { (feet) }\end{array}$} & \multicolumn{3}{|c|}{ Water level } \\
\hline $\begin{array}{l}\text { Local USGS } \\
\text { identification; } \\
\text { well name or number; } \\
\text { and owner }\end{array}$ & $\begin{array}{c}\text { Standard } \\
\text { USGS } \\
\text { ident ification }\end{array}$ & & & & & Date & $\begin{array}{l}\text { Depth } \\
\text { (feet below } \\
\text { (and surface) }\end{array}$ & $\begin{array}{l}\text { Measure- } \\
\text { ment } \\
\text { method }\end{array}$ \\
\hline
\end{tabular}

Las Vegas Valley Water District Measurements

\begin{tabular}{|c|c|c|c|c|c|c|c|}
\hline $\begin{array}{c}212 \text { S19 E60 O4DAB1 } \\
\cdots \text { / NEV. DIV. OF } \\
\text { FORESTRY }\end{array}$ & 361939115154801 & I & 2,454 & 780 & $\cdots$ & $\begin{array}{l}06-25-86 \\
09-10-86 \\
12-16-86 \\
03-03-87\end{array}$ & $\begin{array}{l}70.57 \\
74.91 \\
72.29 \\
71.5\end{array}$ \\
\hline $\begin{array}{l}212 \text { \$19 E60 09BCC1 } \\
\cdots \text { / J.P. GOUMAND }\end{array}$ & 361843115161001 & $\mathbf{U}$ & 2,510 & 830 & $\cdots$ & $\begin{array}{l}02-25-86 \\
06-25-86 \\
09-10-86 \\
12-16-86 \\
03-03-87\end{array}$ & $\begin{array}{l}150.63 \\
155.13 \\
158.20 \\
153.59 \\
153.0\end{array}$ \\
\hline $\begin{array}{l}212 \text { S19 E60 } 12081 \\
\cdots \text { / E. LAMB }\end{array}$ & 361806115122701 & H & 2,350 & 240 & 80 & $\begin{array}{l}02-25-86 \\
06-26-86 \\
09-10-86 \\
12-16-86 \\
03-03-87\end{array}$ & $\begin{array}{l}128.92 \\
124.99 \\
125.59 \\
125.75 \\
126.10\end{array}$ \\
\hline $\begin{array}{c}212 \text { \$19 E60 2280DI } \\
\cdots / \text { B. REID }\end{array}$ & 361703115150601 & $\mathbf{P}$ & 2,360 & 400 & 200 & $\begin{array}{l}02-25-86 \\
06-25-86 \\
09-10-86 \\
12-16-86 \\
03-18-87\end{array}$ & $\begin{array}{l}134.36 \\
168.80 \\
177.00 \\
137.13 \\
132.72\end{array}$ \\
\hline $\begin{array}{l}212 \text { \$19 E60 24CBC1 } \\
- \text { / D.L. OHAIR }\end{array}$ & 361655115132101 & H & 2,315 & 380 & 210 & $\begin{array}{l}02-25-86 \\
06-25-86 \\
09-10-86 \\
12-16-86 \\
03-03-87\end{array}$ & $\begin{array}{l}122.93 \\
153.32 \\
158.03 \\
127.33 \\
127.62\end{array}$ \\
\hline $\begin{array}{c}212 \text { S19 E60 298DO1 } \\
\cdots / \text { P. EDMUNDSON }\end{array}$ & 361613115171401 & H & 2,530 & 303 & - & $\begin{array}{l}06-25-86 \\
09-10-86 \\
12-16-86 \\
03-18-87\end{array}$ & $\begin{array}{l}176.53 \\
187.07 \\
179.68 \\
178.62\end{array}$ \\
\hline $\begin{array}{c}212519 \text { E61 210081 } \\
\text { REGIONAL PARK / } \\
\text { CITY N.L.V. }\end{array}$ & 361626115090701 & $\mathbf{u}$ & 2,160 & 1,300 & 50 & $\begin{array}{l}02-25-86 \\
06-26-86 \\
09-19-86 \\
12-17-86 \\
03-17-87\end{array}$ & $\begin{array}{l}32.44 \\
33.88 \\
34.38 \\
33.72 \\
33.28\end{array}$ \\
\hline $\begin{array}{l}212 \$ 19 \text { E62 290DBA1 } \\
\cdots \text { L. YOFFE }\end{array}$ & 361555115034801 & $\mathbf{u}$ & 1,975 & 300 & 150 & $\begin{array}{l}02-26-86 \\
06-26-86 \\
09-15-86 \\
12-17-86 \\
03-18-87\end{array}$ & $\begin{array}{l}96.80 \\
96.7 \\
97.5 \\
97.5 \\
97.4\end{array}$ \\
\hline $\begin{array}{l}212519 \text { E62 32BBAA1 } \\
\# 2 \text { / USGS }\end{array}$ & 361542115042901 & U & 1,958 & 95 & 91 & $\begin{array}{l}02-26-86 \\
06-26-86 \\
09-15-86 \\
12-17-86 \\
03-17-87\end{array}$ & $\begin{array}{l}92.77 \\
93.02 \\
92.30 \\
91.98 \\
91.83\end{array}$ \\
\hline
\end{tabular}


TABLE 2,--Water-level data for wells measured about quarterly--Cont inued.

\begin{tabular}{|c|c|c|c|c|c|c|c|c|}
\hline \multicolumn{2}{|c|}{ Site designations } & \multirow{2}{*}{$\begin{array}{c} \\
\text { Water } \\
\text { use }\end{array}$} & \multirow{2}{*}{$\begin{array}{l}\text { Land- } \\
\text { surface } \\
\text { alti tude } \\
\text { (feet } \\
\text { above } \\
\text { sea } \\
\text { level) }\end{array}$} & \multirow[b]{2}{*}{$\begin{array}{l}\text { Depth } \\
\text { of } \\
\text { well } \\
\text { (feet) }\end{array}$} & \multirow[b]{2}{*}{$\begin{array}{l}\text { Depth to } \\
\text { shal lowest } \\
\text { opening } \\
\text { (feet) }\end{array}$} & \multicolumn{3}{|c|}{ Water level } \\
\hline $\begin{array}{l}\text { Local USGS } \\
\text { identification; } \\
\text { well name or number; } \\
\text { and owner }\end{array}$ & $\begin{array}{c}\text { Standard } \\
\text { USGS } \\
\text { identification }\end{array}$ & & & & & Date & $\begin{array}{l}\text { Depth } \\
\text { (feet below } \\
\text { land surface) }\end{array}$ & $\begin{array}{l}\text { Measure- } \\
\text { ment } \\
\text { method }\end{array}$ \\
\hline \multirow[t]{2}{*}{$\begin{array}{l}212 \text { S20 E60 11CAAA1 } \\
\# 28 / \text { LVWWD }\end{array}$} & 361303115140301 & $\mathbf{P}$ & 2,287 & 1,003 & 307 & $\begin{array}{l}02-28-86 \\
03-31-86 \\
06-27-86 \\
06-30-86 \\
08-30-86 \\
12-31-86\end{array}$ & $\begin{array}{l}315 . \\
317 . \\
306.67 \\
312 . \\
317 . \\
310 .\end{array}$ & $\begin{array}{l}A \\
A \\
S \\
A \\
A \\
A\end{array}$ \\
\hline & & & & & & $\begin{array}{l}01-09-87 \\
01-31-87 \\
02-28-87 \\
04-30-87\end{array}$ & $\begin{array}{l}321.7 \\
310 . \\
310 . \\
318 .\end{array}$ & $\begin{array}{l}\mathbf{S} \\
\mathbf{A} \\
\mathbf{A} \\
\mathbf{A}\end{array}$ \\
\hline $\begin{array}{l}212 \text { S20 E60 23AABA1 } \\
\# 51 / \text { LVVWD }\end{array}$ & 361143115133801 & $\mathbf{P}$ & 2,270 & 900 & 330 & $\begin{array}{l}02-28-86 \\
03-31-86 \\
04-30-86 \\
12-31-86 \\
01-31-87 \\
02-28-87 \\
03-31-87\end{array}$ & $\begin{array}{l}293 . \\
310 . \\
306 . \\
303 . \\
301 . \\
298 . \\
296 .\end{array}$ & $\begin{array}{l}\hat{A} \\
\hat{A} \\
\hat{A} \\
\hat{A} \\
\hat{A} \\
\hat{A}\end{array}$ \\
\hline \multirow[t]{2}{*}{$\begin{array}{l}212 \text { S20 E60 26DCC1 } \\
\# 24 \text { / LVVWD }\end{array}$} & 361025115133801 & $\mathbf{P}$ & 2,335 & 960 & 400 & $\begin{array}{l}02-28-86 \\
03-31-86 \\
10-31-86 \\
11-30-86 \\
12-18-86 \\
12-31-86\end{array}$ & $\begin{array}{l}370 . \\
368 . \\
390 . \\
387 . \\
391.28 \\
380 .\end{array}$ & $\begin{array}{l}\hat{A} \\
\hat{A} \\
A \\
\hat{A} \\
\mathbf{S} \\
\hat{A}\end{array}$ \\
\hline & & & & & & $\begin{array}{l}01-31-87 \\
02-28-87 \\
03-31-87 \\
04-01-87 \\
04-30-87\end{array}$ & $\begin{array}{l}376 . \\
373 \\
379 \\
379.2 \\
377 .\end{array}$ & $\begin{array}{l}\hat{A} \\
\hat{A} \\
\hat{A} \\
\mathbf{S} \\
\hat{A}\end{array}$ \\
\hline \multirow[t]{2}{*}{$\begin{array}{l}212 \text { S20 E60 35DDA2 } \\
\# 1 A \text { / LVWWD }\end{array}$} & 360940115133701 & $\mathbf{P}$ & 2,300 & 1,006 & 550 & $\begin{array}{l}02-28-86 \\
03-31-86 \\
10-31-86 \\
11-30-86 \\
12-18-86 \\
12-31-86 \\
01-31-87\end{array}$ & $\begin{array}{l}334 . \\
335 . \\
352 . \\
363 . \\
352.24 \\
399 . \\
351 .\end{array}$ & $\begin{array}{l}A \\
A \\
A \\
A \\
S \\
A \\
A\end{array}$ \\
\hline & & & & & & $\begin{array}{l}02-28-87 \\
03-02-87 \\
03-31-87 \\
04-30-87\end{array}$ & $\begin{array}{l}350 . \\
336.55 \\
333 . \\
331 .\end{array}$ & $\begin{array}{l}A \\
S \\
A \\
A\end{array}$ \\
\hline $\begin{array}{l}212 \text { S20 E62 05CAAA1 } \\
\text { WILSHIRE / CITY } \\
\text { N.L.V. }\end{array}$ & 361400115040901 & $\mathbf{P}$ & 1,869 & 1,000 & 500 & $\begin{array}{l}02-26-86 \\
09-16-86 \\
09-19-86 \\
01-09-87 \\
03-17-87\end{array}$ & $\begin{array}{c}96.11 \\
144.82 \\
135.08 \\
94.7 \\
88.9\end{array}$ & $\begin{array}{l}\mathbf{S} \\
\mathbf{S} \\
\mathbf{s} \\
\mathbf{T} \\
\mathbf{T}\end{array}$ \\
\hline $\begin{array}{l}212 \text { S20 E62 15BBAB1 } \\
\text { NELLIS "C" \#12 / } \\
\text { U.S. ARMY CORPS } \\
\text { OF ENGINEERS }\end{array}$ & 361233115021501 & $\mathbf{P}$ & 1,816 & 1,000 & 320 & $\begin{array}{l}02-26-86 \\
09-15-86 \\
01-09-87 \\
03-18-87\end{array}$ & $\begin{array}{c}101.57 \\
128.2 \\
91.96 \\
93.2\end{array}$ & $\begin{array}{l}s \\
\mathbf{r} \\
\mathbf{s} \\
\mathbf{r}\end{array}$ \\
\hline
\end{tabular}


TABLE 2.--Water-level data for wells measured about quarterly--Continued.

\begin{tabular}{|c|c|c|c|c|c|c|c|c|}
\hline \multicolumn{2}{|c|}{ site designations } & \multirow{2}{*}{\multicolumn{2}{|c|}{$\begin{array}{c}\text { Land- } \\
\text { surface } \\
\text { altitude } \\
\text { (feet } \\
\text { above } \\
\text { Water sea } \\
\text { use level) }\end{array}$}} & \multirow[b]{2}{*}{$\begin{array}{l}\text { Depth } \\
\text { of } \\
\text { well } \\
\text { (feet) }\end{array}$} & \multirow[b]{2}{*}{$\begin{array}{l}\text { Depth to } \\
\text { shall lowest } \\
\text { opening } \\
\text { (feet) }\end{array}$} & \multicolumn{3}{|c|}{ Water level } \\
\hline $\begin{array}{l}\text { Local USGS } \\
\text { identification; } \\
\text { well name or number; } \\
\text { and owner }\end{array}$ & $\begin{array}{c}\text { Standard } \\
\text { USGS } \\
\text { identification }\end{array}$ & & & & & Date & $\begin{array}{l}\text { Depth } \\
\text { (feet below } \\
\text { land surface) }\end{array}$ & $\begin{array}{c}\text { Measure- } \\
\text { ment } \\
\text { method }\end{array}$ \\
\hline $\begin{array}{l}212 \text { S20 E62 188ABA1 } \\
\text { GOWAN \#2 / CITY } \\
\text { N.L.V. }\end{array}$ & 361243115052501 & $\mathbf{P}$ & 1,847 & 700 & 350 & $\begin{array}{l}02-26-86 \\
01-09-87 \\
03-18-87\end{array}$ & $\begin{array}{l}61.58 \\
62.41 \\
60.34\end{array}$ & $\begin{array}{l}\mathbf{S} \\
\mathbf{S} \\
\mathbf{S}\end{array}$ \\
\hline $\begin{array}{l}212 \text { S20 E62 } 268 B C C 1 \\
\cdots / F . \text { PURDY }\end{array}$ & 361100115011901 & H & 1,900 & 330 & 160 & $\begin{array}{l}02-26-86 \\
06-27-86 \\
01-07-87 \\
03-16-87\end{array}$ & $\begin{array}{l}154.34 \\
145.40 \\
145.0 \\
146.3\end{array}$ & $\begin{array}{l}\mathbf{S} \\
\mathbf{S} \\
\mathbf{T} \\
\mathbf{T}\end{array}$ \\
\hline 212 S20 E62 34СABB1 & 360952115020701 & $U$ & 1,740 & 100 & -- & $\begin{array}{l}02-26-86 \\
06-26-86 \\
09-11-86 \\
01-07-87 \\
03-16-87\end{array}$ & $\begin{array}{l}39.80 \\
39.62 \\
39.73 \\
38.86 \\
38.32\end{array}$ & $\begin{array}{l}\mathbf{S} \\
\mathbf{S} \\
\mathbf{S} \\
\mathbf{S} \\
\mathbf{S}\end{array}$ \\
\hline 212521 E60 O1DBB1 & 360847115125301 & $U$ & 2,261 & 190 & $\cdots$ & $\begin{array}{l}02-26-86 \\
06-25-86 \\
09-10-86\end{array}$ & $\begin{array}{l}111.04 \\
119.46 \\
110.45\end{array}$ & $\begin{array}{l}\mathbf{S} \\
\mathbf{S} \\
\mathbf{S}\end{array}$ \\
\hline $\begin{array}{l}212 \text { S21 E61 O4DDBA1 } \\
\# 4 \text { I USGS }\end{array}$ & 360846115091401 & $\mathbf{U}$ & 2,042 & 500 & $\cdots$ & $\begin{array}{l}02-26-86 \\
06-27-86 \\
09-11-86 \\
12-18-86 \\
03-20-87\end{array}$ & $\begin{array}{l}28.14 \\
34.40 \\
36.74 \\
29.98 \\
29.40\end{array}$ & $\begin{array}{l}\mathbf{S} \\
\mathbf{S} \\
\mathbf{S} \\
\mathbf{S} \\
\mathbf{S}\end{array}$ \\
\hline 212 S21 E61 04DDBA2 & 360846115091402 & $U$ & 2,042 & 20 & 16 & $\begin{array}{l}02-26-86 \\
06-25-86 \\
09-12-86 \\
03-20-87\end{array}$ & $\begin{array}{l}7.34 \\
7.39 \\
7.86 \\
7.23\end{array}$ & $\begin{array}{l}\mathbf{S} \\
\mathbf{S} \\
\mathbf{S} \\
\mathbf{S}\end{array}$ \\
\hline $\begin{array}{cl}212 & \text { S21 E61 O9BBBB1 } \\
\# 3 \text { / USGS }\end{array}$ & 360838115101801 & $U$ & 2,075 & 25 & 21 & $\begin{array}{l}02-26-86 \\
06-26-86 \\
09-12-86 \\
12-18-86 \\
03-18-87\end{array}$ & $\begin{array}{l}12.32 \\
12.7 \\
10.37 \\
11.77 \\
12.06\end{array}$ & $\begin{array}{l}\mathbf{S} \\
\mathbf{S} \\
\mathbf{S} \\
\mathbf{S} \\
\mathbf{S}\end{array}$ \\
\hline $\begin{array}{l}212 \text { S21 E61 15DDDD1 } \\
\cdots \text { / CLARK CO. }\end{array}$ & 360701115081301 & $U$ & 2,000 & 24.1 & 21.1 & $\begin{array}{l}02-28-86 \\
06-26-86 \\
09-12-86 \\
12-29-86 \\
03-02-87\end{array}$ & $\begin{array}{l}15.44 \\
15.16 \\
15.27 \\
14.36 \\
15.75\end{array}$ & $\begin{array}{l}\mathbf{S} \\
\mathbf{S} \\
\mathbf{S} \\
\mathbf{S} \\
\mathbf{S}\end{array}$ \\
\hline $\begin{array}{l}212 \text { \$21 E61 26DDBB1 } \\
\cdots \text { / CLARK CO. }\end{array}$ & 360522115072101 & $u$ & 2,010 & 29.7 & 25.7 & $\begin{array}{l}02-26-86 \\
06-25-86 \\
09-11-86 \\
12-29-86 \\
03-02-87\end{array}$ & $\begin{array}{l}16.90 \\
16.00 \\
14.63 \\
14.91 \\
15.5\end{array}$ & $\begin{array}{l}\mathbf{S} \\
\mathbf{S} \\
\mathbf{S} \\
\mathbf{S} \\
\mathbf{T}\end{array}$ \\
\hline $\begin{array}{l}212 \text { S21 E61 30DABB1 } \\
\text { / S.T. HALL }\end{array}$ & 360518115112101 & $-\cdot$ & 2,225 & 304 & $\cdots$ & $\begin{array}{l}02-26-86 \\
06-27-86 \\
09-12-86 \\
12-30-86 \\
03-02-87\end{array}$ & $\begin{array}{l}157.38 \\
171.90 \\
173.02 \\
171.2 \\
171.1\end{array}$ & $\begin{array}{l}\mathbf{S} \\
\mathbf{S} \\
\mathbf{S} \\
\mathbf{T} \\
\mathbf{T}\end{array}$ \\
\hline $\begin{array}{l}212 \text { S21 E62 19AABA1 } \\
-- \text { / LVWO }\end{array}$ & 36065111505050 & $\mathbf{u}$ & 1,840 & 23 & $\cdots$ & $\begin{array}{l}02-26-86 \\
06-25-86 \\
09-12-86 \\
12-29-86 \\
03-02-87\end{array}$ & $\begin{array}{l}12.24 \\
12.29 \\
12.27 \\
12.23 \\
11.11\end{array}$ & $\begin{array}{l}\mathbf{S} \\
\mathbf{S} \\
\mathbf{S} \\
\mathbf{S} \\
\mathbf{S}\end{array}$ \\
\hline
\end{tabular}


TABLE 2.--Water-level data for wells measured about quarterly--Cont inued.

\begin{tabular}{|c|c|c|c|c|c|c|c|c|}
\hline \multicolumn{2}{|c|}{ Site designations } & \multirow{2}{*}{\multicolumn{2}{|c|}{$\begin{array}{c}\text { Land- } \\
\text { surface } \\
\text { altitude } \\
\text { (feet } \\
\text { above } \\
\text { Water sea } \\
\text { use level) }\end{array}$}} & \multirow[b]{2}{*}{$\begin{array}{l}\text { Depth } \\
\text { of } \\
\text { well } \\
\text { (feet) }\end{array}$} & \multirow[b]{2}{*}{$\begin{array}{l}\text { Depth to } \\
\text { shallowest } \\
\text { opening } \\
\text { (feet) }\end{array}$} & \multicolumn{3}{|c|}{ Water level } \\
\hline $\begin{array}{l}\text { Local USGS } \\
\text { identification; } \\
\text { well name or number; } \\
\text { and owner }\end{array}$ & $\begin{array}{l}\text { Standard } \\
\text { USGS } \\
\text { identification }\end{array}$ & & & & & Date & $\begin{array}{l}\text { Depth } \\
\text { (feet below } \\
\text { land surface) }\end{array}$ & $\begin{array}{l}\text { Measure- } \\
\text { ment } \\
\text { method }\end{array}$ \\
\hline $\begin{array}{l}212 \text { S21 E62 } 27 C C C B 1 \\
\cdots \text { / NEVADA POWER }\end{array}$ & 360509115023001 & $u$ & 1,665 & 360 & $\cdots$ & $\begin{array}{l}02-28-86 \\
06-25-86 \\
09-11-86 \\
12-24-86 \\
03-02-87\end{array}$ & $\begin{array}{l}18.88 \\
19.42 \\
19.97 \\
19.12 \\
18.95\end{array}$ & $\begin{array}{l}S \\
S \\
S \\
S \\
S\end{array}$ \\
\hline $\begin{array}{l}212 \text { S21 E63 298881 } \\
\# 1 \text { / USGS }\end{array}$ & 360832115060201 & $u$ & 1,590 & 80 & 76 & $\begin{array}{l}02-28-86 \\
06-25-86 \\
09-11-86 \\
12-22-86 \\
03-17-87\end{array}$ & $\begin{array}{l}17.81 \\
18.06 \\
18.05 \\
19.89 \\
20.35\end{array}$ & $\begin{array}{l}\text { S } \\
\text { S } \\
\text { S } \\
\text { S } \\
\text { S }\end{array}$ \\
\hline $\begin{array}{l}212 \text { S22 E60 23BBD1 } \\
\cdots \text { / J. HORNYAK }\end{array}$ & 360118115141101 & - & 2,545 & 500 & 410 & $\begin{array}{l}02-26-86 \\
09-10-86\end{array}$ & $\begin{array}{l}424.56 \\
462.80\end{array}$ & $\begin{array}{l}\mathbf{S} \\
\mathbf{S}\end{array}$ \\
\hline $\begin{array}{l}212 \text { S22 E61 12AAAD1 } \\
\cdots / \text { J. BROWN }\end{array}$ & 360321115060001 & $\mathbf{P}$ & 2,020 & 500 & 160 & $\begin{array}{l}02-28-86 \\
06-25-86 \\
09-11-86 \\
12-17-86 \\
03-02-87\end{array}$ & $\begin{array}{l}39.39 \\
63.25 \\
66.00 \\
38.52 \\
35.6\end{array}$ & $\begin{array}{l}S \\
S \\
S \\
S \\
Y\end{array}$ \\
\hline $\begin{array}{l}212 \text { S22 E61 18CACD1 } \\
--/ \mathrm{J} \text {. KNEZ }\end{array}$ & 360156115120501 & -- & 2,356 & 360 & -- & $09-10-86$ & 258.45 & $\mathbf{s}$ \\
\hline $\begin{array}{l}212 \text { S22 E61 208AD1 } \\
-- \text { / E. CLOVER }\end{array}$ & 360112115104301 & $\cdots$ & 2,287 & 210 & $-\cdot$ & $\begin{array}{l}02-28-86 \\
06-26-86 \\
09-11-86 \\
12-18-86 \\
02-27-87\end{array}$ & $\begin{array}{l}197.09 \\
203.78 \\
200.05 \\
199.3 \\
199.04\end{array}$ & $\begin{array}{l}\text { S } \\
\mathbf{S} \\
\mathbf{S} \\
\mathbf{S} \\
\mathbf{S}\end{array}$ \\
\hline $\begin{array}{l}212 \text { S22 E61 220BA1 } \\
-- \text { / R. SOWER }\end{array}$ & 360114115082701 & $H$ & 2,205 & 300 & 200 & $\begin{array}{l}02-28-86 \\
09-11-86 \\
03-18-87\end{array}$ & $\begin{array}{l}132.56 \\
134.11 \\
136.7\end{array}$ & $\begin{array}{l}\mathbf{S} \\
\mathbf{S} \\
\mathbf{S}\end{array}$ \\
\hline 212 S22 E61 23CBD1 & 360104115080001 & $\cdots$ & 2,190 & 143 & -- & $\begin{array}{l}02-28-86 \\
06-27-86 \\
09-11-86\end{array}$ & $\begin{array}{l}122.62 \\
124.15 \\
125.03\end{array}$ & $\begin{array}{l}\mathbf{S} \\
\mathbf{S}\end{array}$ \\
\hline 212 S22 E61 290CDB1 & 360002115103801 & -- & 2,275 & 300 & -- & $\begin{array}{l}02-26-86 \\
06-27-86 \\
09-11-86 \\
12-19-86 \\
02-27-87\end{array}$ & $\begin{array}{l}124.54 \\
125.18 \\
125.55 \\
125.6 \\
124.4\end{array}$ & $\begin{array}{l}S \\
S \\
S \\
1 \\
T\end{array}$ \\
\hline $\begin{array}{l}212 \text { S22 E62 08CBDC1 } \\
\text { / PARADISE V. } \\
\text { COUNTRY CLUB }\end{array}$ & 360241115044001 & I & 1,982 & 712 & 565 & $01-08-87$ & 135.25 & $\mathbf{S}$ \\
\hline $\begin{array}{c}212 \text { S23 E61 03BCC1 } \\
\text { / SKY HARBOR } \\
\text { AIRPORT }\end{array}$ & 361136115101401 & $\mathbf{u}$ & 2,375 & 650 & 220 & $\begin{array}{l}02-28-86 \\
06-27-86 \\
09-11-86 \\
12-19-86\end{array}$ & $\begin{array}{l}207.4 \\
207.37 \\
208.59 \\
208.65\end{array}$ & $\begin{array}{l}T \\
S \\
S \\
S\end{array}$ \\
\hline
\end{tabular}


TABLE 3.--Daily mean water levels for three wells equipped with recorders.

Hydrographs are shown on plate 2

[--, no data available; water levels are in feet below land surface.]

Site 11: Local identification 212 S20 E61 310DCB1; standard identification 360938115112801 . Well depth, 1,250 feet.

\begin{tabular}{|c|c|c|c|c|c|c|c|c|c|}
\hline \multirow[b]{2}{*}{ DAY } & \multicolumn{9}{|c|}{1986} \\
\hline & APR. & MAY & JUNE & JULY & AUG. & SEP. & OCT. & NOV. & DEC. \\
\hline 1 & -- & 177.91 & 194.02 & 203.56 & 208.99 & 211.06 & 211.37 & 208.05 & 203.25 \\
\hline 2 & $\cdots$ & 177.84 & 194.99 & 203.59 & 209.13 & 211.14 & 211.32 & 207.65 & 197.74 \\
\hline 3 & $\cdots$ & 177.83 & 195.34 & 203.79 & 209.24 & 211.28 & 210.82 & 207.40 & 196.88 \\
\hline 4 & $\cdots$ & 177.85 & 196.11 & 203.90 & 209.40 & 211.28 & 210.02 & 207.29 & 196.66 \\
\hline 5 & -. & 178.31 & 196.41 & 204.22 & 209.35 & 211.41 & 209.99 & 207.03 & -. \\
\hline 6 & 176.83 & 178.91 & 196.62 & 204.60 & 209.27 & 211.35 & 210.10 & 206.57 & 196.04 \\
\hline 7 & 177.32 & 179.28 & 196.97 & 204.61 & 209.78 & 211.46 & 210.13 & 206.52 & 195.81 \\
\hline 8 & 177.77 & 179.41 & 197.37 & 204.37 & 210.40 & 211.53 & 210.15 & 206.45 & 195.70 \\
\hline 9 & 177.71 & 179.41 & 197.49 & 204.45 & 210.54 & 211.49 & 210.26 & 206.12 & 194.03 \\
\hline 10 & 177.65 & 179.54 & 197.65 & 204.94 & 210.73 & 211.61 & 210.25 & 205.99 & 192.74 \\
\hline 11 & 177.51 & 179.70 & 198.24 & 205.62 & 210.82 & 211.72 & 210.15 & 205.77 & 192.40 \\
\hline 12 & 177.79 & 179.81 & 198.43 & 205.67 & 210.55 & 211.75 & 210.26 & 205.60 & -. \\
\hline 13 & 177.92 & 180.18 & 198.46 & 205.95 & 210.80 & 211.77 & 210.33 & 205.41 & $-\cdot$ \\
\hline 14 & 177.81 & 181.36 & 199.03 & 206.16 & 210.95 & 211.90 & 210.34 & 204.45 & -- \\
\hline 15 & 177.81 & 181.81 & 200.81 & 206.30 & 211.05 & 211.16 & 210.31 & 204.24 & -. \\
\hline 16 & 177.93 & 181.83 & 201.00 & 205.97 & 211.20 & 212.01 & 210.16 & 203.94 & -- \\
\hline 17 & 178.11 & 181.77 & - & 205.81 & 211.34 & 211.75 & 209.75 & 203.74 & -. \\
\hline 18 & 178.31 & 181.63 & -. & 206.56 & 211.60 & 211.59 & 209.57 & 203.51 & -. \\
\hline 19 & 178.14 & $181.5 / 4$ & -- & 207.02 & 211.67 & 211.67 & 209.86 & 203.68 & $\cdots$ \\
\hline 20 & 178.00 & 182.07 & $\cdots$ & 206.99 & 210.27 & 211.75 & 210.02 & 203.46 & $\cdots$ \\
\hline 21 & 177.96 & 182.43 & $\cdots$ & 206.97 & 210.12 & 211.83 & 210.03 & 203.39 & $\cdots$ \\
\hline 22 & 177.87 & 182.59 & $\cdots$ & 207.22 & 210.22 & 211.73 & 209.69 & 203.35 & $\cdots$ \\
\hline 23 & 177.86 & 182.67 & - & 207.37 & 210.19 & 211.60 & 209.81 & 203.31 & -- \\
\hline 24 & 177.90 & 182.54 & $\cdots$ & 207.54 & 210.31 & 211.51 & 209.40 & 203.37 & $-\cdot$ \\
\hline 25 & 178.04 & 182.51 & -. & 207.79 & 210.37 & 211.56 & 209.32 & 201.53 & -- \\
\hline 26 & 178.10 & 182.67 & -- & 207.90 & 210.69 & 211.90 & 209.21 & 203.29 & - \\
\hline 27 & 177.84 & 182.69 & -- & 208.08 & 210.79 & 211.80 & 208.76 & 203.62 & - \\
\hline 28 & 177.88 & 182.87 & 203.05 & 208.32 & 210.71 & 211.73 & 208.50 & 203.33 & -. \\
\hline 29 & 177.89 & 183.54 & 203.11 & 208.52 & 210.84 & 211.71 & 208.43 & 203.37 & $-\cdot$ \\
\hline 30 & 177.86 & 183.66 & 203.22 & 208.61 & 210.93 & 211.69 & 208.26 & 203.53 & -. \\
\hline 31 & $\cdots$ & 184.47 & - & 208.77 & 210.99 & - & 208.55 & - & -- \\
\hline MEAN & $\cdots$ & 181 & $\cdots$ & 206 & 210 & 212 & 210 & 205 & -. \\
\hline $\operatorname{MAX}$ & $\cdots$ & 184.47 & $\therefore$ & 208.77 & 211.67 & 212.01 & 211.37 & 208.05 & -. \\
\hline MIN & -. & 177.83 & -. & 203.56 & 208.99 & 211.06 & 208.26 & 201.53 & -. \\
\hline
\end{tabular}


TABLE 3.--Daily mean water levels for three wells equipped with recorders on plate 2--Continued

$$
\text { [--, no data available; water levels are in feet below land surface.] }
$$

Site 13: Local identification 212 S20 E62 03BBCC1; standard identification 361449115022901. Well depth, 1,397 feet.

\begin{tabular}{|c|c|c|c|c|c|c|c|c|c|c|c|c|c|c|}
\hline \multirow[b]{2}{*}{ DAY } & \multicolumn{9}{|c|}{1986} & \multicolumn{5}{|c|}{1987} \\
\hline & APR. & MAY & JUNE & JULY & AUG. & SEP. & OCT. & NOV. & DEC. & JAN. & FEB. & MAR. & APR. & MAY \\
\hline 1 & $\cdots$ & 88.78 & 89.45 & 98.46 & 101.46 & 98.78 & 96.56 & 94.18 & 92.26 & 90.74 & 89.72 & 88.94 & 88.36 & 88.15 \\
\hline 2 & -- & 88.92 & 90.00 & 98.78 & 100.96 & 99.05 & 96.46 & 94.11 & 92.18 & 90.72 & 89.66 & 88.95 & 88.34 & 88.11 \\
\hline 3 & $\cdots$ & 88.96 & 90.44 & 98.89 & 100.59 & 99.16 & 96.40 & 94.04 & 92.14 & 90.67 & 89.67 & 88.98 & 88.24 & 88.13 \\
\hline 4 & $\cdots$ & 89.06 & 90.62 & 98.67 & 101.09 & 99.16 & 96.30 & 94.00 & - & 90.72 & 89.69 & 88.96 & 88.25 & - \\
\hline 5 & -. & 88.93 & 91.45 & 98.30 & 101.31 & 99.16 & 96.19 & 93.90 & 91.93 & 90.73 & 89.70 & 88.90 & 88.28 & $\cdots$ \\
\hline 6 & $\cdots$ & 88.84 & 92.09 & 98.16 & 101.31 & 98.81 & 96.10 & 93.81 & 91.88 & 90.77 & 89.72 & 88.85 & 88.38 & -- \\
\hline 7 & -. & 88.81 & 92.23 & 98.35 & 100.87 & 98.63 & 95.98 & 93.77 & 91.85 & 90.75 & 89.65 & 88.80 & 88.40 & - \\
\hline 8 & - & 88.76 & 92.20 & 98.85 & 100.73 & 98.47 & 95.89 & 93.78 & 91.84 & 90.74 & 89.57 & 88.78 & 88.52 & -. \\
\hline 9 & 88.71 & 88.74 & 92.63 & 99.21 & 100.70 & 98.37 & 96.08 & 93.73 & 91.79 & 90.71 & 89.57 & 88.86 & 88.60 & -. \\
\hline 10 & 88.68 & 88.66 & 93.18 & 99.51 & 100.14 & 98.49 & 96.06 & 93.65 & 91.76 & 90.64 & 89.44 & 88.86 & 88.55 & $-\cdot$ \\
\hline 11 & 88.67 & 88.63 & 93.75 & 99.53 & 100.53 & 98.64 & 95.89 & 93.58 & -. & 90.61 & 89.47 & 88.83 & 88.39 & $\cdots$ \\
\hline 12 & 88.62 & 88.65 & 94.02 & 99.88 & 100.15 & 98.73 & 95.81 & 93.50 & $\cdots$ & 90.54 & 89.46 & 88.78 & 88.41 & - \\
\hline 13 & 88.71 & 88.87 & 94.29 & 100.16 & 99.97 & 98.46 & 95.73 & 93.40 & 91.58 & 90.49 & 89.37 & 88.70 & 88.40 & -. \\
\hline 14 & 88.70 & 88.91 & 94.50 & 100.39 & 100.18 & 98.30 & 95.60 & 93.35 & 91.52 & 90.44 & 89.35 & 88.62 & 88.33 & $\cdots$ \\
\hline 15 & 88.61 & 88.85 & 94.43 & 100.58 & 99.80 & 98.15 & 95.51 & 93.30 & 91.51 & 90.34 & 89.27 & 88.56 & 88.37 & - \\
\hline 16 & 88.67 & 88.86 & 94.68 & 100.72 & 99.23 & 98.02 & 95.41 & 93.22 & 91.45 & 90.31 & 89.29 & 88.58 & 88.32 & -. \\
\hline 17 & 88.78 & 88.86 & 95.19 & 100.86 & 99.12 & 97.94 & 95.26 & 92.96 & 91.40 & 90.32 & 89.27 & 88.60 & 88.19 & - \\
\hline 18 & 88.78 & 88.74 & 95.57 & 101.00 & 100.06 & 97.77 & 95.15 & 92.81 & 91.39 & 90.28 & 89.23 & 88.52 & 88.11 & $\cdots$ \\
\hline 19 & 88.79 & 88.66 & 95.96 & 101.14 & 100.66 & 97.63 & 95.06 & 92.80 & 91.31 & 90.20 & 89.30 & 88.50 & 88.19 & $\cdots$ \\
\hline 20 & 88.70 & 88.84 & 96.37 & 101.30 & 100.20 & 97.52 & 94.99 & 92.97 & 91.27 & 90.22 & 89.31 & 88.60 & 88.19 & -. \\
\hline 21 & 88.63 & 89.13 & 96.51 & 101.04 & 99.64 & 97.45 & 94.86 & 92.95 & 91.29 & 90.17 & 89.25 & 88.61 & 88.14 & -- \\
\hline 22 & 88.74 & 89.69 & 96.85 & 100.41 & 99.36 & 97.41 & 95.07 & 92.89 & 91.25 & 90.07 & 89.19 & 88.58 & 88.06 & $\cdots$ \\
\hline 23 & 88.76 & 89.63 & 97.08 & 100.29 & 99.16 & 97.26 & 95.13 & 92.85 & 91.18 & 89.99 & 89.04 & 88.60 & 88.19 & - \\
\hline 24 & 88.72 & 89.57 & 97.44 & 100.66 & 99.02 & 97.09 & 94.98 & 92.72 & 91.12 & 90.00 & 89.06 & 88.55 & 88.21 & $\cdots$ \\
\hline 25 & 88.67 & 89.42 & 97.71 & 100.94 & 99.12 & 96.99 & 94.84 & 92.62 & 91.08 & 90.01 & 89.05 & 88.54 & 88.15 & $\cdots$ \\
\hline 26 & 88.69 & 89.30 & 97.94 & 101.14 & 99.57 & 96.92 & 94.71 & 92.59 & 91.05 & 89.94 & 89.02 & 88.53 & 88.12 & -. \\
\hline 27 & 88.68 & 89.21 & 98.22 . & 101.31 & 99.87 & 96.83 & 94.60 & 92.53 & 90.98 & 89.85 & 89.05 & 88.50 & 88.06 & -. \\
\hline 28 & 88.59 & 89.31 & 98.47 & 101.42 & 99.77 & 96.72 & 94.50 & 92.42 & 90.97 & 89.78 & 89.03 & 88.47 & 88.03 & $\cdots$ \\
\hline 29 & 88.56 & -- & 98.13 & 101.63 & 99.72 & 96.93 & 94.41 & 92.31 & 90.95 & 89.78 & $\cdots$ & 88.47 & 88.22 & $\cdots$ \\
\hline 30 & 88.55 & 89.39 & 97.94 & 101.80 & 99.33 & 96.75 & 94.28 & 92.34 & 90.88 & 89.72 & -. & 88.46 & 88.24 & $\cdots$ \\
\hline 31 & -- & 89.37 & - & 101.84 & 99.00 & $-\cdot$ & 94.22 & -- & 90.80 & 89.70 & $-\cdot$ & 88.39 & -. & $\cdots$ \\
\hline MEAN & -- & $\cdots$ & 94.6 & 100 & 100 & 98.0 & 95.4 & 93.2 & $\cdots$ & 90.3 & 89.4 & 88.7 & 88.3 & $-\cdot$ \\
\hline $\max$ & -. & -. & 98.47 & 101.84 & 101.46 & 99.16 & 96.56 & 94.18 & -- & 90.77 & 89.72 & 88.98 & 88.60 & $-\cdot$ \\
\hline MIN & -. & $\cdots$ & 89.45 & 98.16 & 99.00 & 96.72 & 94.22 & 92.31 & $\cdots$ & 89.70 & 89.02 & 88.39 & 88.03 & -. \\
\hline
\end{tabular}


TABLE 3.--Daily mean water levels for three wells equipped with recorders--Continued

$$
\text { [--, no data available; water levels are in feet below land surface.] }
$$

Site 16: Local identification 212 S21 E61 27DDBC1; standard identification 360518115082401. Well depth, 1,200 feet.

\begin{tabular}{|c|c|c|c|c|c|c|c|c|c|c|c|c|c|}
\hline \multirow[b]{2}{*}{ DAY } & \multicolumn{9}{|c|}{1986} & \multicolumn{4}{|c|}{1987} \\
\hline & APR. & MAY & JUNE & JULY & AUG. & SEP. & OCT. & NOV. & DEC. & JAN. & FEB. & MAR. & APR. \\
\hline 1 & $\cdots$ & 23.51 & 26.74 & 28.86 & 29.89 & 30.44 & 29.16 & 27.23 & 25.22 & 23.07 & 21.59 & - & $\cdots$ \\
\hline 2 & -- & 23.62 & 26.75 & 28.91 & 29.97 & 30.41 & 29.03 & 27.17 & 25.13 & 23.06 & 21.55 & -- & $\cdots$ \\
\hline 3 & -- & 23.72 & 26.74 & 28.99 & 30.05 & 30.42 & 28.97 & 27.10 & 25.11 & 23.00 & 21.51 & - & $\cdots$ \\
\hline 4 & $\cdots$ & 23.81 & 26.77 & 29.04 & - & 30.47 & 28.90 & 27.10 & 25.07 & 22.92 & 21.54 & -. & 22.07 \\
\hline 5 & $\cdots$ & 23.94 & 26.86 & 29.16 & 30.37 & 30.48 & 28.83 & 27.04 & 24.97 & 22.82 & 21.58 & $\cdots$ & 22.12 \\
\hline 6 & $\cdots$ & 23.99 & 26.94 & 29.31 & 30.49 & 30.48 & 28.78 & 26.93 & 24.91 & 22.84 & 21.59 & -. & 22.18 \\
\hline 7 & - & 24.05 & 27.01 & 29.43 & 30.51 & 30.52 & 28.76 & 26.89 & 24.80 & 22.75 & 21.59 & -- & 22.21 \\
\hline 8 & -. & 24.17 & 27.07 & 29.52 & 30.54 & 30.53 & 28.69 & 26.90 & 24.73 & 22.73 & 21.56 & $-\cdot$ & 22.28 \\
\hline 9 & 22.21 & 24.31 & 27.17 & 29.58 & 30.63 & 30.46 & 28.64 & 26.86 & 24.67 & 22.70 & 21.56 & $\cdots$ & 22.34 \\
\hline 10 & 22.19 & 24.36 & 27.31 & 29.68 & 30.68 & 30.49 & 28.54 & 26.82 & 24.61 & 22.60 & 21.56 & $\cdots$ & 22.38 \\
\hline 11 & 22.23 & 24.48 & 27.43 & 29.72 & 30.65 & 30.53 & 28.40 & 26.78 & 24.52 & 22.50 & 21.58 & $\cdots$ & 22.42 \\
\hline 12 & 22.27 & 24.66 & 27.54 & 29.78 & 30.59 & 30.50 & 28.37 & 26.70 & 24.45 & 22.40 & 21.62 & -. & 22.45 \\
\hline 13 & 22.31 & 24.82 & 27.58 & 29.89 & 30.53 & 30.52 & 28.31 & 26.63 & 24.36 & 22.27 & 21.58 & $\cdots$ & 22.61 \\
\hline 14 & 22.36 & 24.93 & 27.63 & 29.95 & 30.58 & 30.49 & 28.24 & 26.56 & 24.27 & 22.19 & 21.64 & $\cdots$ & 22.73 \\
\hline 15 & 22.34 & 25.03 & 27.69 & 29.91 & 30.58 & 30.48 & 28.12 & 26.55 & 24.21 & 22.05 & 21.62 & $\cdots$ & 22.82 \\
\hline 16 & 22.34 & 25.22 & 27.79 & 29.86 & 30.61 & & & 26.49 & & 22.06 & & $\cdots$ & 22.87 \\
\hline 17 & 22.35 & 25.46 & 27.91 & 29.93 & 30.71 & 30.32 & 27.97 & 26.42 & 24.05 & 22.11 & 21.74 & $\cdots$ & 22.89 \\
\hline 18 & 22.45 & 25.56 & 27.97 & 30.00 & 30.76 & 30.22 & 27.92 & 26.30 & 23.98 & 22.12 & 21.70 & $\cdots$ & 22.87 \\
\hline 19 & 22.60 & 25.62 & 28.03 & 30.09 & 30.76 & 30.14 & 27.88 & 26.20 & 23.89 & 22.05 & 21.73 & $\cdots$ & 22.91 \\
\hline 20 & 22.75 & 25.65 & - & 30.13 & 30.81 & 30.13 & 27.84 & 26.11 & 23.79 & 22.06 & 21.76 & $-\cdot$ & 23.05 \\
\hline 21 & 22.76 & 25.67 & 28.23 & 30.00 & 30.80 & 30.14 & 27.76 & 25.95 & 23.77 & 21.98 & 21.77 & $\cdots$ & 23.14 \\
\hline 22 & 22.77 & 25.65 & 28.32 & 29.91 & 30.88 & 30.12 & 27.63 & 25.89 & 23.70 & 21.89 & 21.80 & $\cdots$ & 23.19 \\
\hline 23 & 22.80 & 25.66 & 28.42 & 29.81 & 30.94 & 29.98 & 27.57 & 25.83 & 23.60 & 21.76 & 21.69 & $-\cdot$ & $\cdots$ \\
\hline 24 & 22.86 & 25.82 & 28.39 & 29.70 & 30.92 & 29.82 & 27.52 & 25.71 & 23.52 & 21.81 & 21.72 & -. & $\cdots$ \\
\hline 25 & 22.90 & 25.92 & 28.41 & 29.51 & 30.88 & 29.73 & 27.49 & 25.57 & 23.46 & 21.84 & $\cdots$ & $\cdots$ & $\cdots$ \\
\hline 26 & 23.04 & 26.04 & 28.49 & 29.48 & 30.80 & 29.68 & 27.44 & 25.54 & & & $\cdots$ & -- & $\cdots$ \\
\hline 27 & 23.15 & 26.16 & 28.54 & 29.50 & 30.69 & 29.63 & 27.41 & 25.50 & 23.34 & 21.70 & $\cdots$ & $\cdots$ & $\cdots$ \\
\hline 28 & 23.20 & 26.34 & 28.61 & 29.57 & 30.51 & 29.54 & 27.43 & 25.40 & 23.32 & 21.60 & $\cdots$ & $\cdots$ & -- \\
\hline 29 & 23.27 & 26.54 & 28.68 & 29.63 & 30.42 & 29.48 & 27.34 & 25.27 & 23.31 & 21.60 & - & - & - \\
\hline 30 & 23.39 & 26.68 & 28.75 & 29.72 & 30.38 & 29.37 & 27.26 & 25.28 & 23.24 & 21.56 & - & - & - \\
\hline 31 & $\cdots$ & 26.77 & -. & 29.84 & 30.39 & - & 27.21 & - & 23.14 & 21.56 & $\cdots$ & $\cdots$ & $\cdots$ \\
\hline MEAN & $\cdots$ & 25.1 & $\cdots$ & 29.6 & $\cdots$ & 30.2 & 28.1 & 26.4 & 24.2 & 22.2 & $\cdots$ & $\cdots$ & $\cdots$ \\
\hline $\max$ & $\cdots$ & 26.77 & $\cdots$ & 30.13 & $\cdots$ & 30.53 & 29.16 & 27.23 & 25.22 & 23.07 & $\cdots$ & $-\cdot$ & $\cdots$ \\
\hline MIN & $\cdots$ & 23.51 & $\cdots$ & 28.86 & $\cdots$ & 29.37 & 27.21 & 25.27 & 23.14 & 21.56 & $\cdots$ & $\cdots$ & $\cdots$ \\
\hline
\end{tabular}


Plume, R.W., 1984, Ground-water conditions in Las Vegas Valley, Clark County, Nevada--Part I, hydrogeologic framework: U.S. Geological Survey Open-File Report 84-130, 25 p.

Rush, F.E., 1968, Index of hydrographic areas in Nevada: Nevada Division of Water Resources Information Report 6, 38 p. 